\title{
Quasi-Empirical Fictionalism
}

as an approach to the

\section{Philosophy of Geometry}

By Scott Waygood 


\section{Contents}

Chapter 1. Introduction.....................3

Chapter 2. Epistemology...................14

Chapter 3. Ontology.......................42

Chapter 4. Geometry.......................78

Chapter 5. Conclusion.....................127

Bibliography............................135 
Chapter 1. Introduction 


\section{$\S 1.1$ Abstract}

The central claim of this thesis is that geometry is a quasi-empirical science based on the idealisation of the elementary physical operations that we actually perform with pen and paper. This conclusion is arrived at after searching for a theory of geometry that will not only explain the epistemology and ontology of mathematics, but will also fit with the best practices of working mathematicians and, more importantly, explain why geometry gives us knowledge that is relevant to physical reality.

We will be considering all the major schools of thought in the philosophy of mathematics. Firstly, from the epistemological side, we will consider apriorism, empiricism and quasi-empiricism, finding a Kitcherian style of quasi-empiricism to be the most attractive. Then, from the ontological side, we will consider Platonism, formalism, Kitcherian ontology, and fictionalism. Our conclusion will be to take a Kitcherian epistemology and a fictionalist ontology. This will give us a kind of quasiempirical-fictionalist approach to mathematics.

The key feature of Kitcher's thesis is that he placed importance on the operations rather than the entities of arithmetic. However, because he only dealt with arithmetic, we are left with the task of developing a theory of geometry along Kitcherian lines. I will present a theory of geometry that parallels Kitcher's theory of arithmetic using the drawing of straight lines as the most primitive operation. We will thereby develop a theory of geometry that is founded upon our operations of drawing lines. Because this theory is based on our line drawing operations carried out in physical reality, and is the idealisation of those activities, we will have a connection between mathematical geometry and physical reality that explains the predictive power of geometry in the real world.

Where Kitcher uses the Peano postulates to develop his theory of arithmetic, I will use the postulates of projective geometry to form the foundations of operational geometry. The reason for choosing projective geometry is due to the fact that by taking it as the foundation, we may apply Klein's Erlanger programme and build a theory of geometry that encompasses Euclidean, hyperbolic and elliptic geometries. 
The final question we will consider is the problem of conventionalism. We will discover that investigations into conventionalism give us further reason to accept the Kitcherian quasi-empirical-fictionalist approach as the most appealing philosophy of geometry available. 


\section{§1.2 Desiderata}

I have identified four key areas that any good philosophical theory of mathematics must address. A good theory will explain the epistemology of mathematics giving a clear account of the nature of mathematical knowledge. A good theory will also make clear its ontological commitments and offer a credible description of the nature of mathematical entities. A good theory also needs to fit with the best practices of working mathematicians. Finally, and what turns out to be the crucial factor in choosing a good overall theory, a good theory must be able to account for the success of mathematics in terms of our ability to use mathematics to make accurate descriptions, and predications, about the states of affairs in physical reality. In this section I will briefly outline these four key desiderata.

\section{Epistemology}

We will assume from the outset that everyone has some mathematical knowledge (and some people have a lot of mathematical knowledge). This assumption naturally leads to several questions about the nature of mathematical knowledge. How do we obtain mathematical knowledge? Do we gain this knowledge through interaction with our physical environment in an a posteriori fashion, or do we gain this knowledge through purely a priori intuitions?

Given that we have some mathematical knowledge, some mathematical statements must be true, and so we may ask the question, what is the nature of these mathematical truths? Are they contingent or necessary, synthetic or analytic, are they timelessly true, and, striking to the heart of the issue, in virtue of what are mathematical statements true?

There are several competing views on this topic, and we shall be considering three of them. The first, and traditionally most popular, view is apriorism. On this view mathematical knowledge is gained independently of our experiences with the physical world, either through our intuition, or through our knowledge of purely logical 
implications. However, we will find several very good reasons to reject this theory. The next view we will look at will be empiricism, the view that mathematics is the most general of all empirical sciences. This view is somewhat incompatible with the actual practices of working mathematicians and we will finally arrive at a quasiempirical view of mathematical knowledge, and conclude that this is the best option available.

\section{Ontology}

Mathematical entities such as numbers, sets, points and lines are widely considered to be abstract entities, therefore we need an account of their ontological status. We will be considering four distinct views on the question of the ontological nature of mathematical entities. The first will be the Platonism, which argues that mathematical entities are real mind independent non spatio-temporal entities. Secondly, we will consider formalism which claims that there are no mathematical entities beyond symbols on a page, and hence such entities are unworthy of inclusion in our ontological list of things that exist. Thirdly, having already accepted the quasiempirical view of mathematics as the preferred epistemological theory, we will explore the ontological stance this theory leads us to. Finally, we will consider fictionalism, which claims that mathematical objects are fictional entities that (do not) exist in the same sense that Frodo Baggins (does not) exist. We will also find that the fictionalist view is compatible with quasi-empiricism and that the two views may be combined to make a good overall theory of mathematics.

\section{Best Practice}

A philosophical theory of mathematics must explain what it is that mathematicians do when they are doing mathematics. A good theory should fit with our actual mathematical practices and our actual mathematical experiences. Whatever answer that philosophers come up with to explain what mathematics actually is, must conform to the mathematician's opinion of their own best practice. This is to say that a good philosophy of mathematics should be descriptive rather than prescriptive. 
An example of an approach that should be rejected on these grounds would be constructivism, as it denies the law of excluded middle and labels as unmathematical a large portion of what mathematicians study. We will therefore not be considering this view.

\section{Utility}

Mathematics has a very good track record in making accurate predictions about the world. I can use arithmetic to predict how many apples will be on the table after so many have been added to, and so many have been taken away from, a collection already on the table. I can use the theorem of Pythagoras to determine the diagonal of a sports field without having to measure it given that I already know the lengths of each side. How is this possible? How does mathematics relate to the world?

One of the main problems with many philosophical accounts of mathematics is that if you subscribe to these accounts, then the success of mathematics becomes a miracle. I am seeking an account that will not make the success of mathematics in dealing with physical reality a mystery, but one that will give an account of this success. This is the key issue I intend to address, and will be the key factor in accepting or rejecting the various philosophical accounts that we will be looking at. 


\section{$\S 1.3$ Geometry Overview}

In this section I will give a very brief outline of the geometrical theories that we will be considering. I will give this outline from a historical perspective because of the epistemological significance that will later be placed on the historical chain of events that has led to our current knowledge of geometry.

The systematic cataloguing and analysis of geometric operations in the ancient world began in Mesopotamia several thousand years ago; it was developed further in ancient Greece, and culminated in Euclid's Elements of Geometry written in about 300 BCE. Euclid's Elements was so comprehensive and well organised that it was soon recognised for its general excellence and replaced all other more inferior works, becoming the textbook for geometry for over two millennia. "It has appeared in more editions than any other work other than the bible. It has been translated into countless languages and has been continuously in print in one country or another nearly since the beginning of printing." (Katz 1998, p. 58)

One of the most significant features of Euclid's Elements is that "it provided ... a model for how pure mathematics should be written with well thought-out axioms, precise definitions, carefully stated theorems, and logically coherent proofs." (Katz 1998, p. 58) In other words Euclid provided a model for the axiomatic method, a method that "endowed geometry with a level of certainty never previously attained by any other science," (Reichenbach 1957, p. 1) and is still used today in all branches of mathematics and logic. The significance of the axiomatic method is that the certainty of the axioms is transferred to the theorems, and conversely the epistemological question of the truth of the theorems may be reduced to the question of the truth of the axioms. This method not only provides a model for how pure mathematics should be performed but also a model to which any pure science should aspire. "Geometry thus became the prototype of a demonstrable science," (Reichenbach 1957, p. 1) and "the aim of every scientific student of every subject was to bring his knowledge of that subject into a form as perfect as that which geometry had attained." (Clifford 1956, p. 552) 
It is from Euclid's Elements that Kant arrived at the view that geometric truths are true a priori. If the postulates are self evidently true, and the theorems are all logical consequences of the postulates, then it follows that the theorems may be considered to be true a priori. This view will be examined in more detail in Section 2.2.

The one blemish on Euclidean geometry was considered to be the $5^{\text {th }}$ postulate that implied the existence of one and only one parallel to a given line through a point not on that line. This does not appear to be as self evident as the other postulates as it requires us to continually produce a line. Many mathematicians throughout the ages, most famously Proclus (410-485 C.E.) and Gauss (1777-1855), have tried and failed to prove that this postulate could be derived from the other four. Gauss even tried to determine empirically whether this postulate holds true. Gauss also experimented with developing non-Euclidean geometry, but didn't publish these investigations because he believed they would not be accepted by the academic community of the time.

The next significant chapter in the development of geometry was the study of perspective drawing by renaissance artists such as Durer (1471-1582) and de Vinci (1452-1519). This work was then developed into the theory of projective geometry by Desargues (1591-1661). The work of Desargues was largely ignored during his lifetime, probably due to his use of non-standard notation and novel terminology that few could understand, and to the increasing popularity of the analytical geometry being developed by Descartes (1596-1650) around the same time. In fact, the work of Desargues disappeared out of circulation completely until the nineteenth century when a manuscript copy made by one of Desargues' pupils was discovered by mathematician Michel Chasles in a second hand bookstore.

Monge (1746-1818) carried out work similar to that of Desargues and developed a theory of descriptive geometry. Poncelet (1788-1867), a student of Monge and an officer in Napoleon's army, was captured during the invasion of Russia and spent a year in a Russian prison. There he reconstructed from memory all that he had learned from Monge, and he proceeded to create a theory of synthetic projective geometry. Although I will be considering the analytic treatment of geometry in Section 4.3, it is 
the synthetic side that I am primarily interested in because of the way it fits with the philosophical account of mathematics that I am supporting.

During the early part of the nineteenth century the Hungarian, Bolyai (1802-1860), and the Russian, Lobatschewsky (1792-1856) independently and almost simultaneously discovered that a self-consistent geometry could be developed by replacing Euclid's $5^{\text {th }}$ postulate with an axiom postulating the existence of at least two parallels to a line through a point not on that line. Later, Klein proved the consi stency of these systems by showing a correspondence between theorems of nonEuclidean geometry with theorems of Euclidean geometry. The result was the first proof of consistency in the history of mathematics. (The proof of the consistency of Euclidean geometry was given by Hilbert using a reduction to arithmetic.) After these investigations the mathematical legitimacy of these systems was established beyond question.

The geometry developed by Bolyai and Lobatschewsky is known as hyperbolic geometry. In this geometry, given a line and a point not on it, there are two parallel lines and an infinite number of ultra-parallel lines to the line going through the point, and the angle sum of any triangle is less than two right angles.

Riemann (1826-1866) developed an alternative theory of geometry from a different angle. He considered a geometry to be a manifold on which we may establish various metric relationships. Generalising from the work by Gauss on the curvature of surfaces, Riemann defined a measure of curvature intrinsic to a manifold that may be described in the metric. He also showed that for a body to be able to move freely in a manifold, the measure of curvature would have to be constant. Taking this foundation to geometry we may develop either Euclidean geometry, if we take the measure of curvature to be everywhere zero, or hyperbolic geometry if we take the measure of curvature to be negative. And this may be done without making assumptions about the nature of parallel lines.

Helmholtz (1821-1894) took the theory of Riemann one step further and showed how to develop a third kind of geometry, elliptic geometry, by taking the measure of curvature to be a positive constant. This new geometry is similar to geometry on a 
sphere. There are no parallels, every pair of lines meet, and the angle sum of a triangle is greater than two right angles.

The revolution in mathematics caused by the development of non-Euclidean geometries was on a par with the Copernican revolution in astronomy. Mathematics could no longer be considered a priori as the curvature of physical space could only be determined by empirical investigations. Furthermore, the Riemannian manifolds were used by Einstein as the mathematical foundation of his theory of relativity, and experiments were carried out in 1919 by Eddington and Cottingham involving the passage of light rays originating from distant stars and passing close to the sun during a solar eclipse. These experiments were heralded as providing empirical evidence that physical space is in fact non-Euclidean.

With the development of various axiomatic systems and the plurality of geometries a new answer to the epistemological problem was recognised, it was no longer considered to be within the domain of mathematics to decide which axioms are true, and the implicational nature of mathematics was highlighted. Mathematicians study which statements can be derived as theorems from each axiomatic system, therefore mathematicians can only conclude that if the axioms are true, then these theorems will also be true, and not that these theorems are in fact true. This is the view of mathematics put forward by Reichenbach and the other logical positivists in response to the developments in mathematics and theoretical physics outlined above.

The situation with multiple geometries is, however, more interesting than just having a collection of alternative geometries to choose from, the various geometries form a hierarchy that has interesting philosophical implications. After the foundations of geometry were seemingly shattered by the development of non-Euclidean geometries by Bolyai, Lobatchewsky, Riemann and Helmholtz, the foundations of geometry were reformulated in a unifying way by Klein and Cayley placing projective geometry as the basis for all geometries, both Euclidean and non-Euclidean. Russell, when studying these developments, came to the conclusion that it was projective geometry, and not Euclidean geometry, that provided the "a priori properties of any form of externality." (Kline, forward to Russell (1956).) Although my conclusion is strikingly 
different from Russell's, I do share the belief that projective geometry ought to play a central role in forming the foundations of geometry. 
Chapter 2. Epistemology 


\section{\$2.1 Introduction}

In this chapter I will be considering three epistemological schools of thought on the nature of mathematical knowledge. The first is apriorism. There have been many philosophers of mathematics who have held the belief that mathematical statements in general, and geometrical statements in particular, are a priori, most notably Kant. I, however, will not be considering the views of Kant as they pre-date the creation/discovery of the non-Euclidean geometries which are the subject of this thesis. Instead I will focus on Hempel as the flag bearer for the arpiorism of geometry. The reasons for this choice are that (1) Hempel presents a very strong and appealing argument, and (2) Hempel is a key player in the logical empiricist movement and uses his aprioristic approach to mathematics in general to form the foundation of a conventionalist view of geometry, and one of the aims of this thesis is to offer a more attractive alternative to conventionalism.

First, I will present the apriorism of Hempel in a non-critical manner, and then we will consider the objections to apriorism raised by Kitcher. These objections will lead us to reject apriorism and thus we will find the need to look for a different view of mathematical knowledge. The second theory we will consider is empiricism. The doctrine of empiricism with respect to mathematical knowledge was put forward by Mill, and although it is a very appealing theory we will find that it is too crude to adequately explain mathematical knowledge.

The final view we will consider is quasi-empiricism. We will find in the quasiempiricism of Kitcher a view that mathematical knowledge is the idealisation of empirical operations. This view places empirical investigations, both of our own and of our distant ancestors, at the foundation of mathematical knowledge. We will find that this view, though built on the empiricism of Mill, is comprehensive enough to give a clear account of mathematical knowledge that satisfies the criteria we are seeking. 


\section{§2.2 Apriorism}

The defining feature of an aprioristic account of mathematical knowledge is the notion that no empirical evidence is required to test whether mathematical statements are true or not, and that mathematical truths are arrived at independently of experience. For the apriorist mathematical truths are among the most certain of all knowledge, are analytically true, and hence are of the same ilk as "all bachelors are unmarried".

Hempel presents a strong argument for apriorism which centres on the implicational nature of mathematics. Hempel believes that statements of empirical science are synthetic and a posteriori. Such statements can always be reviewed and amended as new evidence comes to light. Statements of mathematics, according to Hempel, are analytic statements which can be determined with theoretical certainty, but convey no factual information. I will outline Hempel's arguments for his aprioristic view of mathematics and then consider Kitcher's arguments against such a stance.

The traditional aprioristic view of geometrical truth was to assign certain propositions the label "self-justified" or "self-evident", and state that these propositions may be automatically counted as pieces of knowledge. The propositions which are given this privileged epistemological role are the axioms of a system, and given that their truth is self-evident, they must be true independently of experience, and are hence considered a priori truths. The theorems are logical consequences of the axioms, and the deduction of theorem from axiom is carried out independently of experience, therefore the theorems must be a priori truths also.

To the pre-Lobatchewskian mathematician this view is very satisfying. Once Euclid's axioms have been accepted as self-evidently true, the truth of all the theorems may then be demonstrated by following the proofs as laid out in the Elements. However, the argument for the self-evident nature of mathematical truth based on the selfevidence of mathematical axioms is untenable, and this is not the sort of apriorism that Hempel supports. Firstly, the plurality of geometries has forced us to acknowledge that there is no one collection self-evident axioms. Secondly, not all 
geometric axioms are even contenders for the title of "self-evident truths". Consider the axiom of parallels, this has never been accepted as being self evident, and this can be seen from the fact that so many mathematicians over the centuries, from Proclus to Gauss, have attempted to prove it.

An even worse theory is that mathematical truths in general do not require factual evidence because they are all self-evident. Hempel, though an apriorist himself, quickly dismisses this thesis on the following grounds:

- Many mathematical theorems are hard to establish, even for experts.

- Many of the interesting results, especially in abstract set theory and topology are counter intuitive.

- The existence of mathematical conjectures such as Goldbach's that remain undecided.

- "Self-evident" is subjective. What one person regards as being self evident, another might not. This does not seem a satisfactory basis to establish the objective validity of mathematical propositions.

Hempel claims that axioms may not be considered as self evident truths, not because they are not self evident, but because they are incapable of being either true or false. The correct way to conceive of axioms, according to Hempel, is as statements which are devoid of factual content, and hence are not capable of being either true or false

"As a consequence, geometry cannot be said to assert the truth of its postulates, since the latter are formulated in terms of concepts without any specific meaning; indeed, for this very reason, the postulates themselves do not make any specific assertion which could possibly be called true or false! In the terminology of modern logic, the postulates are not sentences, but sentential functions with the primitive concepts as variable arguments. - This point also shows that the postulates of geometry cannot be considered as 'self-evident truths,' because where no assertion is made, no self-evidence can be claimed." (Hempel 1945, p. 12)

Hempel's views on the epistemology of mathematics stem from his belief that mathematics is a collection of axiomatic systems in which theorems are logically 
deduced from the postulates, as is the case for the systems of geometry under consideration. He highlights the implicational nature of mathematics and claims that it is not within the domain of mathematics to decide which axioms are true. Mathematicians study which statements can be derived as theorems for each axiomatic system, therefore mathematicians can only conclude that if the axioms are true, then a particular set of theorems will also be true, and not that these theorems are in fact true.

"Thus, each mathematical theorem can be cast into the form

$$
\left(P_{1}, P_{2} . P_{3} \ldots P_{n}\right) \rightarrow T
$$

Where the expression on the left is the conjunction (joint assertion) of all the postulates, the symbol on the right represents the theorem in its customary formulation, and the arrow expresses the relation of logical implication or entailment. Precisely this character of mathematical theorems is the reason for their peculiar certainty and necessity." (Hempel 1945, p. 9)

Therefore the theorem of Pythagoras, for example, correctly conceived, is not simply the familiar statement about the squares of sides of right-angled triangles, but a conditional statement that has as its antecedent the conjunction of the Euclidean postulates and as its consequence the statement about the squares of sides of rightangled triangles.

However, to say that mathematical statements are true by definition in the same way that "all bachelors are unmarried" is true is an oversimplification. In an axiomatic system, unlike a standard definition, the definitions do not determine the meanings of the definienda in a unique fashion. Furthermore, in actual practice we usually leave our primitive terms undefined.

"For the rigorous development of a mathematical theory proceeds not simply from a set of definitions but rather from a set of non-definitional propositions which are not proved within the theory; these are postulates or axioms of the theory. They are formulated in terms of certain basic or primitive concepts for which no definitions are provided within the theory." (Hempel 1964, p. 369) 
However, statements such as "all bachelors are unmarried" and mathematical statements share certain important characteristics: they can be shown to be true (validated) simply by analysing the terms which occur in them with no empirical evidence required. Such statements are classified as analytic and may be considered true a priori, which indicates that their truth is logically independent of, or logically prior to, any experiential evidence.

In any logical deduction the conclusion must be contained in the premises. Therefore theorems offer us nothing objectively or theoretically new as compared with the postulates, though they may offer something psychologically new in the sense that we were not aware that the theorems were implicitly contained in the postulates.

\footnotetext{
"We may, therefore, say that logical deduction - which is the one and only method of mathematical proof - is a technique of conceptual analysis: it discloses what assertions are concealed in a given set of premises, and it makes us realize to what we committed ourselves in accepting those premises." (Hempel 1945, p. 9)
}

Hempel claims that it is unnecessary to connect any meaning to the primitive terms. The terms carry certain connotations because they relate to familiar geometric figures, but we could quite easily replace the terms "point" and "line" with terms like "object of kind 1" and "object of kind 2" and the terms "incidence" and "collineation" with terms like "relation of kind 1" and "relation of kind 2". Using these terms a competent mathematician with no knowledge of the customary geometric connotations could derive all the theorems of the system given the axioms. (In fact, the projective plane of order three has application to statistical research where the terms "lines" and "points" are replaced by other terms.)

"We see therefore that indeed no specific meaning has to be attached to the primitive terms in an axiomatized theory; and in a precise logical presentation of axiomatized geometry the primitive concepts are accordingly treated as socalled logical variables.” (Hempel 1945, p. 12) 
Therefore, according to Hempel, mathematical theorems are devoid of factual content and thus are incapable of being either true or false. The best we can say is that a theorem represents a valid inference of the sort outlined above. On this view theorems are merely sentences of what Hempel and the logical positivists term "linguistic frameworks". Statements will be accepted if they can be proved to follow logically from the axioms, but they may not be correctly labelled as "true" in the empirical sense because they have no factual content. However, theorems are "true" in the analytical sense because they have been derived through deductive reasoning, and are therefore, according to Hempel, true a priori.

"A mathematical truth is irrefutably certain just because it is devoid of factual or empirical content. Any theorem of geometry, therefore, when cast into the conditional form described earlier, is analytic in the technical sense of logic, and thus true $a$ priori; i.e. its truth can be established by means of the formal machinery of logic alone, without any reference to empirical data." (Hempel 1945, pp. 9-10)

To sum up the apriorist's stance, Apriorism used to be the dominant view of mathematical knowledge, however, this was before the revolution of mathematical ideas led by Gauss, Lobatchewsky and Bolyai. However, the traditional apriorist stance based on the idea that the axioms are self-evidently true, and the theorems are logical consequences of the axioms is untenable in a world that embraces the plurality of geometries.

"The fact that these different types of geometry have been developed in modern mathematics shows clearly that mathematics cannot be said to assert the truth of any particular set of geometric postulates; all that pure mathematics is interested in, and all that it can establish, is the deductive consequences of given sets of postulates and thus the necessary truth of ensuing theorems relatively to the postulates under consideration." (Hempel 1945, p. 11)

However, Hempel has reformulated the epistemology of geometry in a way that maintains apriorism by claiming that mathematics is a collection of axiomatic systems 
in which the primitive terms are undefined. The axioms are non-factual statements and the theorems are implicational statements which may be tested for validity, but not for empirical truth (or falsehood). He sums up his views as follows:

"If $P$ be the conjunction of the postulates for a given theory, then the proof of a proposition $T$ of that theory consists in deducing $T$ from $P$ by means of the principles of formal logic. What is established by the proof is therefore not the truth of $T$, but rather the fact that $T$ is true provided the postulates are. But since both $P$ and $T$ contain certain primitive terms of the theory, to which no specific meaning is assigned, it is not strictly possible to speak of the truth of either $P$ or $T$; it is therefore more adequate to state the point as follows: If a proposition $T$ is logically deduced from $P$, then every specific interpretation of the primitives which turns all the postulates of $P$ into true sentences, will also render $T$ a true statement." (Hempel 1964, p. 380)

This sounds all very reasonable. Kitcher, however, believes that there is an underlying flaw in this kind of conception of mathematical knowledge. He points out that Hempel, as well as Carnap and Nagel, have presupposed an apsychologistic epistemology. This apsychologistic view of mathematical knowledge was popular in the twentieth century and Kitcher cites Russell, Moore, Ayer, C. I. Lewis, R. Chisholm, W. Sellars and K. Lehrer as people who have argued in favour of this sort of apsychologistic apriorist stance. This approach states that the true belief that $P$ counts as knowledge that $P$ "if $P$ is 'connected in the right way' to other propositions which are believed." (Kitcher 1984, p. 14) The emphasis is on logical relations among propositions not psychological relations among mental states. Russell is upfront about this and states that he will be "dealing exclusively with logical, as opposed to psychological points of view." (Russell 1956, p. 57)

In contrast, Kitcher argues for a psychologistic epistemology. On this view we suppose that knowledge is true belief, and that there must be an appropriate explanation about how this belief came about, and that this explanation should be rooted in psychology. 
The first problem with apsychologistic epistemology is that, in accordance with Alvin Goldman's “What Is Justified Belief”, we must have an epistemologically relevant justification for a belief to count as knowledge. Kitcher points out that there are many counter-examples that suggest rejecting apsychologistic epistemology. These examples describe cases where the subject meets the requirements for a true belief to count as knowledge, but our intuition is that the reasons are epistemologically defective, and that the subject fails to know. One colourful example is the "recreational gropings of some deranged neurophysiologist." (Kitcher 1984, p. 15)

The logical positivists, such as Ayer and Schlick, wanted to defend the a priori nature of mathematics without venturing into psychology claiming that $\mathrm{X}$ knows $a$ priori that $P$ if and only if $\mathrm{X}$ believes that $P$ and $P$ is analytically true. However, even if you grant that mathematics is analytic the positivist's view fails. Consider a mathematician who believes a certain, as yet unproven, theorem to be true, works hard and eventually constructs a proof of the theorem. Our intuition would be to say that the mathematician did not know the theorem to be true until she had completed the proof. Yet the positivist's view is that, provided the theorem is analytically true, nothing has changed after the proof has been completed. Even worse, other mathematicians may come to believe theorems "because of dreams, trances, fits of Pythagorean ecstasy, and so forth" (Kitcher 1984, pp. 15-6) and provided the theorem is true, on the apsychologistic view, these beliefs count as knowledge.

There are, of course, ways to strengthen the apsychologistic approach against such claims, but Kitcher believes these moves to be mere epicycles that do not cover the root of the problem.

"Given any apsychologistic condition, we can always tell a new version of our story and thereby defeat the proposal. Our success results from the fact that the mere presence in the subject of a particular belief or of a set of beliefs is always compatible with peculiar stories about causal antecedents." (Kitcher 1984, p. 16)

The key feature of Hempel's view, and that of most apriorist theories, is that mathematical knowledge is gained through proofs. As proofs appear to be carried out 
without reference to experience it seems, on the surface at least, plausible to contend that proofs give us a priori knowledge. Therefore, mathematical knowledge must be a priori. We will now examine more closely the nature of mathematical proofs from a psychologistic angle to see if they do, in fact, engender a priori knowledge.

Kitcher claims that the mere existence of a proof of a theorem does not provide grounds for claiming that a mathematician knows the theorem, the mathematician must be able to follow the proof in order for the proof to count as a justification for knowledge, and following a proof is a psychological process. Furthermore, we do not have the innate ability to follow mathematical proofs, this is something that we need to be taught. Therefore we may say that to be able to follow a proof, you must have had some experience with mathematical theory, and so the warrant gained by following a proof may not be said to be independent of experience, and hence is not $a$ priori.

Perhaps the most damning argument is that some proofs in mathematics are too long and complicated to be the source of a priori knowledge. When following a long and complicated proof it is reasonable to assume that there might be an error somewhere in the proof that goes unnoticed. In fact, the longer the proof, the bigger the likelihood of there being an error, therefore we cannot be certain that such proofs are correct, and therefore these proofs cannot furnish us with a priori knowledge.

Kitcher claims that psychologistic epistemology does not commit the genetic fallacy of confusing discovery with justification. Consider the case in which "we are originally led to belief in an epistemically defective way but later acquire excellent grounds for our belief." (Kitcher 1984, p. 16) In this case, we first had the mere true belief that $P$ and later had knowledge that $P$. The distinction is found in the processes that led to our state of belief; the former process did not meet the conditions to count as knowledge, whereas the latter did. We may state this in terms of a process that leads to a state of belief and a process which engenders knowledge.

Another problem with proofs is the question of what counts as an acceptable proof. Formal logic gives a clear definition of proof for a system in structural terms: a proof is a sequence of sentences such that each member of the sequence is either an axiom 
of the system or has been derived from previous members of the sequence in accordance with the rules of the system. This is clearly a relative notion, it is a definition relative to a system. The question then arises: what is a proof simpliciter?

Kitcher decides to call those mathematical theories which mathematicians currently accept standard theories. And call formalizations of those theories which logicians would currently accept standard formal theories. Then a proof is a proof in some standard formal theory.

Clearly this definition of what counts as a proof is dependent on what the mathematical community currently accepts, and what it does not accept. This means that what counts as a proof can be revised by successive generations of mathematical communities. This seems historically correct, as if you consider that even some of the proofs in Euclid's Elements are not considered to be rigorous proofs by today's standards. It might even turn out that our descendents decide to relax the rules. But this goes against the idea that proofs should be timeless, something I believe apriorists would want to claim.

"At best, correct or reasonable social practice can determine which sequences are proofs. Yet now we must ask what makes the adoption of a theory or system correct or reasonable.” (Kitcher 1984, p. 37)

Kitcher's primary example of experience that influences mathematical knowledge are cases of social challenges to mathematical statements. These occur when a person widely regarded as an expert in their field challenges what we have taken to be true. Kitcher argues that because mathematics has changed and been modified in the past, "a little imagination will enable us to see how similar modifications might happen to contemporary claims." (Kitcher 1984, p. 91)

"Finally, if the apriorist should insist that social challenges to mathematical statements are somehow unimportant, then it is appropriate to reply by pointing out the social character of most of our knowledge. There is very little that we know without reliance on the testimony and support of others. Even in the case of empirical science, most of the knowledge of each individual is 
based, not on direct experiences, but on the communication of others. Few of us have performed the delicate experiments, and not many more have studied the experimental results. We read that 'experiment has shown that ...' and we are, reasonably, satisfied. Indeed, the happy few who actually adjust the apparatus and watched the instruments are dependent on their colleagues, albeit in different ways. Their knowledge is sustained, in part, by community approval of their techniques and background assumptions." (Kitcher 1984, p. 91)

Kitcher does not want to claim that proofs cannot furnish us with knowledge, but that the knowledge they do furnish us with is not a priori knowledge, (and that proofs are not the only way to gain knowledge in mathematics).

We have seen that the apriorist's account of mathematical knowledge has several real problems. Firstly, if we take an apsychologistic approach to epistemology, then we have the problem that the mere existence of a proof will provide justification for a mathematician knowing a theorem when she might have arrived at the belief that the theorem was true in an epistemologically insufficient way. Secondly, if we take the psychologistic approach then we will demand that the mathematician must be able to follow the proof for the theorem to count as knowledge. However, when the proof is long and complicated there is a reasonable chance that in following it she has missed a mistake, and therefore following such a proof cannot count as a warrant for a priori knowledge. Finally, what counts as an acceptable proof changes over time, and therefore cannot be considered a warrant for a priori knowledge. Therefore we shall reject apriorism and search for a better view. 


\section{§2.3 Empiricism}

Empiricism is the view that mathematics is an empirical science that differs from other empirical sciences only in that its subject matter is more general and its propositions have been tested and confirmed to a greater degree. Hence the empiricists label mathematics as the "most general empirical science". This view is usually associated with the work of John Stuart Mill but was very unpopular in the early twentieth century and was usually only mentioned in passing and quickly rejected. However, in recent decades this thesis has received more positive press and has formed the basis for a revival in empiricism in the philosophy of mathematics.

Mill claimed that the foundation of all sciences, even mathematics, is induction. From the fact that we have observed things occurring in a certain way in the past, we deduce that they will continue to occur in this way in the future. This, according to Mill, is the basis of all scientific knowledge, and Mill asks why mathematics should be considered to be different.

"Why are mathematics by almost all philosophers, and (by some) even those branches of natural philosophy which through the medium of mathematics, have been converted into deductive sciences, considered to be independent of the evidence of experience and observation and characterized as systems of necessary truth?” (Mill 1950, p. 145)

He answers that the certainty of mathematical truth is an illusion brought about by the belief in imaginary objects.

With regard to the definitions of geometry, Mill claims that "there exist no real things exactly conformable to the definitions. There exist no points without magnitude; no lines without breadth, nor perfectly straight; no circles with all their radii exactly equal, nor squares with their angles perfectly right." (Mill 1950, p. 145) He goes on to claim that the existence of such perfect objects is inconsistent with the physical constitution of the universe. 
Mill suggests that in order to get over this difficulty philosophers claim that the objects of geometry exist only in the mind, and then construct an a priori science which is purely mental and has nothing to do with outward experiences. But, claims Mill, surely the mental circle is but a reflection of the physical circle, that the geometrical objects that we hold in our minds are just copies of the ones that we draw on paper. It is interesting that Mill has flipped the Platonistic picture around. Under Plato's theory the objects of the world are reflections of the perfect ideals.

Mill claims that the definitions of geometry are generalisations of physical objects. He states that although it is not exactly true of any one circle that all its radii are equal, it is almost true of all circles that such is the case, therefore "no error of any importance in practice will be incurred by feigning it to be exactly true." (Mill 1950, p. 146) We therefore base our deductive sciences on a foundation of assumptions that are inductively derived and, at best, only approximately true.

Furthermore, Mill claims that the axioms of Euclidean geometry are based on induction. Mill asks us to consider the assumption that two straight lines cannot enclose a space. Surely this is deduced not from our intuition, but as an induction from the evidence of our senses.

A hard-line empiricist, such as Mill, will claim that truths of arithmetic are arrived at through induction on our experiences manipulating medium sized physical objects. For example, we may place three apples on a table, add two more apples, and discover that there are now five apples on the table. We may then use this experiment as confirmation of the hypothesis that $3+2=5$. The more often we perform similar experiments that arrive at the same conclusion, we may say that the hypothesis has been confirmed to a higher and higher degree. The fact that similar experiments have borne out the same result time and time again means that this hypothesis may be accepted as true with a very high degree of certainty. According to this view it is due to the fact that such mathematical statements have been borne out by our experiences in such an overwhelming manner and been confirmed to such a high degree of certainty, that mathematicians have come to regard mathematical statements as qualitatively different from the well confirmed hypotheses of other empirical sciences, and consider them to be certain. The empiricist claims that this is a mistake. 
Some philosophers have simply rejected this kind of view as laughable. Carnap jokes about "trying to find by empirical means the product of seven and eight." (Carnap 1995, pg. 136) However, I believe that the empiricist's stance has to be taken seriously. A serious objection to empiricism is the lack of disconfirming evidence for mathematical propositions. Hempel states that in empirical science a hypothesis may be confirmed or disconfirmed by experimental evidence, and that it is always possible to state the kind of evidence that, if encountered, would cause us to reject the hypothesis.

\begin{abstract}
"Consider now a simple 'hypothesis' from arithmetic: $3+2=5$. If this is actually an empirical generalization of past experiences, then it must be possible to state what kind of evidence would oblige us to concede the hypothesis was not generally true after all.” (Hempel 1964, p. 367)
\end{abstract}

Hempel then goes on to describe an experimental observation where we add two microbes to three microbes only to discover that the result was six microbes. Clearly this would not lead us to conclude that $3+2$ does not equal 5, and I will agree with Hempel that "under no circumstances could the phenomenon just described invalidate the arithmetical proposition in question." (Hempel 1964, p. 368) We would conclude that either we had made a mistake in counting, or that one of the microbes had split into two.

However, this example should not cause us to reject empiricism. Due to the vast number of times that we have observed $3+2$ equalling 5 , it would be bad science to reject the proposition based on a single observation. If $3+2$ resulted in there being 6 objects for a variety of objects and in numerous experiments, then we would have evidence to disconfirm the hypothesis. According to the empiricist the difficulty Hempel has in finding some plausible evidence to disconfirm arithmetic is due to the fact that arithmetic is actually empirically true, and not, as Hempel would have it, due to arithmetic being analytically true.

Hempel asks us to consider another example, the transitivity of identity, i.e., that $a=b$ and $b=c$ implies $a=c$. The example he uses are shades of green, where to the naked 
eye $a$ and $b$ appear the same shade, as do $b$ and $c$, but $a$ and $c$ are clearly different. Would this lead us to reject the logical law? Surely no says Hempel, because "the principle in question is true a priori." (Hempel 1964, p. 369) But this example is also flawed. The experiment merely shows that the naked eye is not a good tool for establishing the identity of two colours.

The main advantage of empiricism is that it gives us a clear account of why mathematics is useful and successful in making predictions about the real world. This success is due to mathematical statements being derived by induction on our experiences in the real world. Another advantage is a clean ontology; we do not have to postulate the existence of any special mathematical entities as mathematical truths are derived from our experiences dealing with objects that we already believe to exist.

However, it is difficult to give a comprehensive account of mathematical knowledge from a hard-line empirical position. There surely must be some room for deductive reasoning within mathematics given that a large part of what mathematicians do is prove theorems deductively. It does not fit with the best practices of mathematicians to say the mathematics is purely an inductive empirical science. Also, it is difficult to see how a purely empirical outlook can explain the more abstract nature of much of mathematics. For many of the theorems of pure mathematics it is difficult to imagine an empirical investigation that would either confirm or disconfirm them. For example, it is difficult to see how a statement such as $\int_{-\infty}^{+\infty} \frac{\sin x}{x}=\pi$ can be based on empirical investigations. This is reason enough for us to reject a hard-line form of empiricism. The quasi-empirical approach outlined below, however, allows us to use empirical investigations to form the foundations of our mathematics, and then allows us to idealise from them to the more abstract branches of mathematics in question. 


\section{§2.4 Quasi-empiricism}

Quasi-empiricism is a view that has emerged relatively recently in the philosophy of mathematics and is proving to be an attractive and popular alternative to apriorism. That this is the case can be seen in the very title of Lakatos' 1986 paper "A Renaissance of Empiricism in the Recent Philosophy of Mathematics." In this paper Lakatos describes empiricism as the "new vogue in the philosophy of mathematics" (Lakatos 1986, p. 31). He goes on to string together a list of quotes from some of the most influential writers in the philosophy of mathematics to show that there has in fact been an undercurrent of empirical thinking brewing in the literature.

Lakatos, Putnam and Kitcher have all put forward quasi-empirical views on the nature of mathematical knowledge. I will briefly outline Lakatos' view, and explain why I believe it to be insufficient. Next, I will consider Kitcher's version of quasiempiricism and present arguments to show that this is the best epistemological theory available to us.

Lakatos, who was influenced by the work of Popper, outlines a doctrine he calls quasi-empiricism. In Lakatos' view, scientific systems can be divided into two categories, Euclidean and quasi-empirical:

\footnotetext{
"Let us call those sentences of a deductive system in which some truth values are initially injected, 'basic statements', and the subset of basic statements which receive the particular value true, 'true basic statements.' Then a system is Euclidean if it is the [deductive] closure of those of its basic statements which are assumed to be true. Otherwise it is quasi-empirical." (Lakatos 1986, pp. 33-4)
}

This means that the flow of truth is the deciding, indeed the defining, feature of a Euclidean or quasi-empirical system. It is important to note that the nature of truth injection into a quasi-empirical system does not need to be by observations of the physical world. "A theory which is quasi-empirical in my sense may be either empirical or non-empirical in the usual sense: it is empirical only if its basic theorems 
are spatio-temporally singular basic statements whose truth values are decided by the time-honoured but unwritten code of the experimental scientist." (Lakatos 1986, p. 34)

The kind of circumstances that would lead one to inject truth into an abstract mathematical system would be, for example, when it is discovered that a mathematical system does what it was designed to do. For example we will see that Klein's analytical system of linear algebra may be used to model Euclidean, hyperbolic or elliptic geometry, therefore we will adopt this model because it is fruitful to do so. In this way a formal theory would be quasi-empirically justified if its theorems correspond to what we already naively believe to be the case.

Putman also puts forward this kind of quasi-empirical theory, and claims that it fits with the practices of mathematicians through the ages. He points out that the "pay off" of accepting a correspondence between lengths and numbers was so great that such a correspondence was assumed to exist long before a proof was constructed. He also considers the quasi-empirical nature of the work of Newton and Leibnitz, which was not made rigorous until much later, and claims that "the real justification of the calculus is its success - its success in mathematics, and its success in physical science.” (Putnam 1986, p. 54)

What this means, however, is that "truth" is injected on the grounds of compliance with an existing theory. Or that "truth" comes down to other qualities such as utility, beauty and simplicity. To me, these grounds are not sufficient to warrant the use of the word "truth". Carnap and the positivists would argue that these theories are neither true nor false because they have no factual contents. To which Lakatos would surely reply that Carnap's criteria is for "empirical truth", not "quasi-empirical truth", which is the topic of discussion.

In brackets Lakatos makes a key point: "Strictly speaking no heuristic falsifier can be more than a rival hypothesis." (Lakatos 1986, p. 41) This highlights the fact that, in geometry, we do have competing hypothesis, but on Lakatos' theory one being true does not imply that the other is false. 
I believe that this kind of quasi-empiricism begs the key question that led us down the road of empiricism, why does mathematics work? This view claims that mathematics is true because it works, but a more informative view would claim that mathematics works because it is true, and then go on to explain how it is that we know it to be true.

While I do not agree with Lakatos in his use of the word "true", he does highlight the fact that in mathematics we do not always begin with a set of principles that we believe to be true and then build a system from those foundations. Often, we have a desired goal in mind for the systems we create, and satisfying that goal is sufficient grounds to accept that theory as useful (but not necessarily true).

Kitcher, who was influenced by Lakatos, proposes a different form of quasiempiricism, and it is this theory that I endorse. The theory has three distinct aspects. Firstly, that our mathematical knowledge is passed down to us through an epistemologically relevant chain from the early mathematicians in the ancient world, via teachers and the academic communities. Secondly, that at the knowledge first acquired in the ancient world at the base of this chain was derived from empirical investigations using sense perception. Thirdly, that our knowledge of mathematics is also partially acquired from our experiences interacting with the physical world. I shall explore the first aspect first.

The first issue in Kitcher's thesis is the claim that our mathematical knowledge is derived, to a large degree, from our teachers, and not from mathematical proofs. Here "teachers" refers to a group comprising of classroom teachers, university lecturers, authors of text books and the academic community as a whole. As a student and teacher of mathematics I can attest that it is in fact true that a large part of each individual's mathematical knowledge can be considered knowledge that has been passed on by teachers, be it through lessons, lectures or books.

"On the approach I have recommended, the knowledge of individual mathematicians is to be explained by the knowledge passed on to them by authorities. ... In many cases, the authority of teachers will entirely account for an individual's mathematical knowledge: some people, probably the majority, only assert mathematical statements which they have not been 
explicitly taught when those statements have been obtained from statements which were explicitly taught by applying rules which were explicitly taught." (Kitcher 1984, p. 95)

Our justification for believing a mathematical theorem may ultimately rest on a logical proof, or even on empirical experimentation carried out centuries ago, but we, personally, need not be aware of this, and certainly do not need to have worked through the proof of every theorem that we know to be true in order for it to count as knowledge. All we need to know is that a person in authority has assured us of its truth, and they in turn were assured by a person in authority, etc. For example, I learned that the theorem of Pythagoras was true five years before I saw the Euclidean proof of it. As a high school student I knew that the theorem was true, and was able to use it to help solve various mathematical problems. I knew that it was true because it is universally accepted by the mathematical community as being true. I had also experimented with drawing triangles and measuring their sides which also helped satisfy me of its truth.

This is not to say that proofs are unimportant in the acquisition of mathematical knowledge. Proofs play an important part in the teaching and learning of mathematics, but their role is more to do with explaining why something is the case, rather than showing that something is in fact the case. I know that something is true, usually, because someone in authority assured me that this is the case, not because I can follow a logical proof of it.

"Mathematical proofs can serve other functions besides that of increasing the certainty of our knowledge. ... Moreover, without making us any more certain that a theorem is true, a proof can show us why it is true: proofs may yield explanatory dividends." (Kitcher 1978, p. 123)

Goedel concurs with this view that the role of a proof is explanatory.

"The role of the alleged 'foundations' is rather comparable to the function discharged, in physical theory, by explanatory hypotheses ... The so-called logical or set-theoretical 'foundation' for number-theory or of any other well- 
established mathematical theory, is explanatory, rather than really foundational.” (Lakatos 1986, p. 32 from Mehlberg 1962, p. 86)

When I studied geometry at university I worked through and memorised the Euclidean proof of the theorem of Pythagoras. What did I gain from this exercise? Working through the proof did not convince me of the theorem's truth, I already knew that it was true, but it did help me to understand why it is true, or at least why the mathematical community accepts it as being true. It also taught me about the implicational nature of Euclidean geometry, from which set of theorems can this theorem be derived, and which assumptions underpin those theorems. Hence I learned something far deeper than the theorem itself: I learned what is required to carry out a Euclidean proof. I gained a good understanding of what is required for something to be accepted by the mathematical community as true, not what is required for something to be true, nor that the theorem of Pythagoras was in fact true.

Putnam also concurs that we may already know, by quasi-empirical/empirical means, that something is true before we succeed in finding a proof for it, and that this should not downgrade the importance of proofs. Putnam believes that proofs and quasiempirical methods should be viewed as complementary. He points out that proofs play the important role of safe-guarding against contradiction, and "will continue to be the primary method of mathematical verification." (Putnam 1986, p. 63)

Once we have accepted that we gain mathematical knowledge from our teachers, it makes sense to ask where our teachers gained their mathematical knowledge from. This is the second part to Kitcher's epistemological theory. Our teachers learned mathematics from their teachers, who learned it from their teachers, and so on back through history. Therefore, our knowledge of mathematics is justified by an epistemological chain that reaches back through history.

Kitcher considers the history of mathematics, and the empirical nature of ancient mathematics, to be central to how we acquire our mathematical knowledge, both as a community, and as individuals. It is clearly the case that the mathematics of the present is influenced by the mathematics of the past. The works of earlier mathematicians are sources of inspiration for modern mathematicians, who extend 
and developed their predecessor's work. Our personal knowledge of mathematics is given to us by the experts of the current mathematical community, the mathematical community received its knowledge from the previous community, and so forth all the way back to people manipulating physical objects and measuring sections of land somewhere, probably in Mesopotamia, several millennia ago.

"I propose that a very limited amount of our mathematical knowledge can be obtained by observations and manipulations of ordinary things. Upon this small basis we erect the powerful general theories of modern mathematics. Responding to the practical problems and methods of the Babylonians, the Greeks developed theories which would systematize the solutions already obtained. Their knowledge was based on the prior empirical knowledge of their predecessors, and, in its turn, it served as the basis for the knowledge of their successors. At each stage in the ensuing story, the knowledge of individuals is generated from the knowledge of teachers, who pass on what the mathematical community has so far learned. The knowledge of the community is itself the product of a long series of episodes, extending back to simple observations with which mathematical knowledge began. For obvious reasons, I shall call a theory of mathematical knowledge constructed along these lines an evolutionary theory of mathematical knowledge." (Kitcher 1984, p. 92)

A point of clarification needs to be made. This theory does not support the idea that any set of beliefs that are widely held by an authoritative group count as knowledge. Receiving knowledge from a teacher is only the start of the story. The teachers themselves must know what they transmit. Therefore we must be able to account for the authority's knowledge, and this is done by looking at how they obtained their knowledge, which leads us along a train of transmission, which, in the case of geometry, originates with the ancient Babylonians making empirical investigations several millennia ago.

It is clear that the knowledge being transmitted is always evolving. Mathematicians take what they have learned, add to it or amend it, and then transmit the updated knowledge to their students. I will not go into a detailed study of how mathematics 
evolves, but only note that mathematics is evolving. Nobody will deny that mathematical knowledge has been passed down from teacher to student in an ever evolving chain. The novelty of Kitcher's approach is that he treats this chain as being epistemologically relevant.

Perhaps the most significant aspect of this thesis is the notion that mathematical knowledge is ultimately founded upon sense perception. In the case of geometry it is safe to assume that the origins of geometry in the ancient world were based on empirical investigations and sense perception. If we assume Kitcher's evolutionary theory of mathematical knowledge to be correct, then we must assume that the geometrical knowledge we have today has an empirical foundation.

The final part of Kitcher's epistemological thesis is that one of the ways that we learn mathematics is to recreate the empirical investigations at the base of the epistemological chain for ourselves. Kitcher writes about a young child using blocks to learn the basic principles of arithmetic. In the same way, I will consider a young child with pencil and ruler learning the fundamental principles of geometry first hand through sense perception and interaction with the physical world.

As a mathematics teacher I am aware that providing tactile activities, activities that involve manipulating physical objects, greatly enhances a student's understanding of fundamental mathematical concepts. Recent literature in the pedagogy of mathematics teaching encourages "hands on" activities. In fact, the Numeracy Project, a recent initiative in New Zealand schools, places great importance on providing hands on activities, and activities that promote learning through sense perception. This approach to the pedagogy of mathematics teaching is advocated in Kitcher's epistemological thesis.

"My solution to the problem of accounting for the origins of mathematical knowledge is to regard our elementary mathematical knowledge as warranted by sense perception. In this way our remote predecessors acquired the first items of mathematical knowledge. We emulate them by using simple observations to provide our children with supplement to the authority of the teacher." (Kitcher 1984, p. 96) 
This means that we may place a far greater level of importance on diagrams as sources of mathematical knowledge than is usually assumed. Kantians supposed that pictorial representations took place in the mind's eye. It would seem that "we do better to resort to external diagrams which are both more readily surveyable in detail and more durable than mental images." (Kitcher 1984, p. 95)
"Perhaps Kant and his successors were right to think that pictorial representation can sometimes be carried out without employing external aids, but they were wrong both to deny the epistemic kinship of imaginative visualisations with sense perception and to overate the extent to which imaginative visualisation is possible." (Kitcher 1984, p. 95)

The next point to be made clear is the nature of mathematical truth. In virtue of what are mathematical theorems true? Kitcher believes that mathematics is true because it correctly describes the structural properties of the world. He finds in Russell a remark that "suggests an answer: arithmetic is concerned with 'the more abstract and general features of the world."' (Kitcher 1978, p. 131) Putnam also supports this view, claiming that "studying how mathematical objects behave might better be described as studying what structures are abstractly possible and what structures are not abstractly possible." (Putnam 1986, p. 50) Thus we are in good company if we assume that mathematics is true in virtue of the structural features of the physical world. And the route to finding out about these features must surely have an empirical element to it.

Perhaps the most attractive feature of the quasi-empirical view is that it is able to address the issue of the utility of mathematics. This is the key area where other views of mathematical knowledge such as Platonism and formalism, fail. Mathematical theorems are useful in providing predictions about the physical world because these theorems are based, ultimately, on empirical investigations.

"We wanted to be able to explain the usefulness of arithmetic. My proposal provides an explanation. Arithmetic is useful because the manipulations we perform on physical objects approximately satisfy the constraints which the 
arithmetical axioms place on the arithmetical operations, just as Euclidean geometry is useful because the contours of physical objects approximately satisfy the constraints which the Euclidean axioms place on geometrical figures.” (Kitcher 1984, p. 133)

Therefore we can see that the quasi-empirical theory of mathematical knowledge satisfies all the criteria we are considering for a good theory of mathematics. It explains how we obtain mathematical knowledge: we learn it from our teachers. It explains the nature of mathematical knowledge: it is a posteriori. It explains the nature of mathematical truth: theorems are true because they are based, ultimately, on accurate empirical investigations. It fits with the practice of actual mathematicians: mathematicians do teach and learn mathematics as part of long historical tradition. And most significantly, it explains the utility of mathematics: mathematics gives us theorems that work for the physical world because these theorems are, ultimately, derived from empirical investigations.

The question remains open, however, as to whether this theory can adequately describe the more abstract areas of pure mathematics. I will show that this theory can in fact account for highly abstract mathematical theories by considering the ideal mathematician in the Section 3.4 on Kitcher's ontology below.

There is also the issue of multiple geometries. As I have stated, the three geometries, Euclidean, hyperbolic and elliptic, give us conflicting theorems for such elementary things as the angle sum of a triangle. It would seem that if geometry has an empirical nature, then only one of these geometries can be true. Yet we want to endorse all three as true. This dilemma will be solved by considering projective geometry as the empirical foundation of geometry, and all other geometries are then built out of this foundation. Thus, while only one geometry can be true of the physical universe, all three may be considered mathematically true. 


\section{\$2.5 Conclusion}

We will be rejecting the view that mathematical knowledge is a priori in favour of the view that it is based, at its foundations, on empirical evidence. After investigation we have found that the apriorist view is untenable. The approach offered by Hempel is untenable because it relies merely on the existence of an analytical proof to warrant knowledge of a given theorem. A better theory would require the mathematician to go through the process of following the proof in order for the proof to provide a warrant for knowledge. However, in following a long and complicated proof we cannot be completely sure that there is no mistake somewhere in the technical details of the proof.

More importantly, what counts as a proof is determined by the mathematical community and varies over time. What counted as a sufficient proof at one time may not count as a sufficient proof at another time. This indicates that a greater significance should be placed on the role of the mathematical community in determining mathematical knowledge. This is exactly what Kitcher's quasiempiricism does.

However, the bottom line is that empiricism just makes more sense. If we are to grant that mathematical geometry can give us knowledge of physical space, then surely it makes more sense to say that this knowledge was arrived at through empirical investigations rather than through pure a priori intuitions. Why should we have intuitive knowledge of the nature of physical space? It makes so much more sense to say that our knowledge of space is derived from empirical investigations.

After considering Mill's empiricism we find it to be too crude and clumsy to give a sufficient epistemology of mathematics. However, using empiricism as a foundation for mathematical knowledge we may build a quasi-empirical theory, such as Kitcher's, which provides an adequate epistemological account of mathematics. We will therefore be supporting Kitcher's quasi-empirical approach to mathematical knowledge. 
The main focus of this thesis will be demonstrating how a Kitcherian quasi-empirical approach to mathematical knowledge, when merged with the fictionalist approach considered in the next chapter, can be used to give a comprehensive and compelling account of geometry. 
Chapter 3. Ontology 


\section{\$3.1 Introduction}

In this chapter we will be considering four different ontological approaches to the philosophy of mathematics. The first approach is Platonism, the belief that mathematics is the study of mind-independent abstract objects. The second is formalism, the notion that mathematics is just the manipulation of meaningless symbols. The third view we will consider is the ontological component of the Kitcherian quasi-empirical approach to mathematics that we have already isolated as having the best epistemological approach. The final category is fictionalism, a view that is not incompatible with the Kitcherian picture. In fact, we will be developing a version of fictionalism that is built on a Kitcherian foundation as our favoured view of mathematics. 


\section{§3.2 Platonism}

Platonists hold the position that mathematical statements are descriptive of a realm of mind-independent abstract objects - such as numbers and sets. Platonism is probably better described as "abstract realism" as it is an ontological doctrine that states that mathematical objects are real, but their existence is non-spatio-temporal and is independent of human minds, and hence can be described as abstract objects. In the Platonistic view of mathematics mathematical facts would be true even if humans had never engaged in the study of mathematics, and furthermore, they would be true even if humans had never existed. In this view mathematical facts are discovered by mathematicians and are timelessly true.

"According to Platonism, mathematical objects are real. Their existence is an objective fact, quite independent of our knowledge of them. Infinite sets, uncountably infinite sets, infinite-dimensioned manifolds, space filling curves - all the members of the mathematical zoo are definite objects, with definite properties, some known, many unknown. These objects are, of course, not physical or material. They exist outside the space and time of physical existence. They are immutable - they were not created, and they will not change or disappear. Any meaningful question about a mathematical object has a definite answer, whether we are able to determine it or not. According to Platonism, a mathematician is an empirical scientist like a geologist; he cannot invent anything, because it is all there already. All he can do is discover." (Davis and Hersh 1981, p. 318)

In this section I will outline three very appealing arguments for considering adopting a Platonistic view of mathematics. Firstly, because Platonism makes sense of the question in virtue of what are mathematical statements true? Secondly, Platonism fits well with the best practices of the working mathematician. Thirdly, that abstract objects seem indispensable if we are to uphold our current scientific theories. Next I will consider the strengths and weaknesses of these arguments. Then I will look at two fundamental problems with Platonism. The first problem is explaining how we come to have knowledge about these abstract entities. The second problem is 
explaining how the study of this realm of abstract entities gives us knowledge that has applications in the study of physical reality. Ultimately I will conclude that these problems are insurmountable and that Platonism is untenable.

Probably the most compelling reason to adopt Platonism is from the assumption I made at the outset, that we have mathematical knowledge. Given that we have mathematical knowledge, then some mathematical statements must be true. Given that some mathematical statements are true, and that our best theory of truth is disquotational, then there must be something in virtue of which they are true. In other words, the singular terms in mathematical statements must refer to things, and it is in virtue of the nature of these things that mathematical statements are true (or false). Now these things cannot be spatio-temporal as the truth of mathematical statements does not seem to depend on the fate of any material objects, and given the infinite nature of mathematics, there simply isn't enough "stuff" in the universe to do the job. They can't be mental entities either for similar reasons. Therefore we are left to conclude that mathematical entities are non-spatio-temporal, mind independent abstract objects.

Another compelling road to Platonism is from considerations of best practice, because this is the way that most mathematicians conceive of mathematics. Kitcher describes Platonism as "currently, the most widely accepted thesis about the nature of mathematical reality" (Kitcher 1984, p. 6). Davis and Hersh agree, but also indicate that Platonism may be hard to defend on philosophical grounds:

"Most writers on the subject would agree that the typical working mathematician is a Platonist on weekdays and a formalist on Sundays. That is, when he is doing mathematics he is convinced that he is dealing with an objective reality whose properties he is attempting to determine. But then, when challenged to give a philosophical account of this reality, he finds it easiest to pretend that he does not believe in it after all." (Davis and Hersh 1981, p. 321)

Maddy makes an argument for Platonism from a Quinean point of view. She uses Putnam's indispensability argument, claiming that our best source of knowledge is 
from science, and that the existence of mathematical objects is implied by our best scientific theories, therefore we should accept the existence of mathematical objects because they are required for science. (Maddy 1990, p. 104) There are interesting conflicts between Quine's naturalism with respect to science and Maddy's naturalism with respect to mathematics, but that is beyond the scope of this essay. This is not the first time that an indispensability argument has been used in the philosophy of mathematics; in 1904 Zermelo argued for the acceptance of the axiom of choice on the grounds that it was "necessary for science." (Putnam 1986, p. 55)

I will consider the indispensability argument first because it is the easiest to dismiss. The argument basically says that we should believe in the existence of mathematical entities because it is our best (scientific) interests to do so. She makes a good case to support the proposition that it is useful to believe in the existence of such objects, but does not make a metaphysical case for their actual existence. To my mind this argument is similar Pascal's wager which shows that it is in our best interests to believe in the existence of God, but does not make a metaphysical case for God's actual existence.

The first argument, that our best theory of truth implies a Platonistic ontology, cannot be cast aside so easily. On the surface this argument gives us good reason to consider Platonism, but if we look a little deeper we will discover that the argument does not hold up under scrutiny. However, if we reject Platonism, which I will show that we are compelled to do, then we are left with the question, in virtue of what are mathematical statements true? We will need to find an alternative answer to this.

An underlying flaw with Platonism was uncovered by Benacerraf in the article "What Numbers Could Not Be" (Benacerraf 1965) and then extended upon in "What Mathematical Truth Could Not Be" (Benacerraf 1998). In these papers he pointed out that it is not enough that true statements be true in virtue of the state of affairs that exists with certain objects, but that our best theory of knowledge demands that there must be a causal link between the knower and the object about which things are known. Given that mathematical objects are non-spatio-temporal and non-mental, then it is hard to see how this causal link requirement can be satisfied. Therefore it is difficult to imagine how we can have knowledge of these abstract objects. 
Furthermore, as Putnam pointed out, "there are real puzzles, especially if one holds a causal theory of reference in some form, as to how one can refer to mathematical objects at all." (Putnam 1986, p. 59)

The standard Platonistic stance on this issue is that we have knowledge of these objects through something called "mathematical intuition". This intuition allows us to know about mathematical objects in much the same way that sense perception allows us to know about physical objects. Platonists argue that through our intuition certain axioms force themselves upon us as being true.

We should grant that intuition plays an important role in our understanding of mathematics, but not the fundamental epistemological role that Platonists would give it. One major problem is that we do not have a good enough theory of neuropsychology to adequately explain mathematical intuition. If we are to adopt a Platonistic view, then we must invoke a psychologistic epistemology powerful enough to explain mathematical intuition, and we simply do not have the science to do so.

Another problem with considering mathematical intuition as a means to access objective abstract entities in a way similar to sense perception of physical objects is the question of why it is that some people seem to find mathematics highly intuitive and are able to "see" facts about mathematical entities with ease, whereas others seem to find mathematics highly counter-intuitive and struggle to understand even the most fundamental truths. Surely if mathematical intuition is analogous to sense perception then we must all have equal access to the raw data, yet this does not seem to be the case. Furthermore, as a mathematics teacher I often find that my intuitive understanding of a mathematical entity is so different from my student's intuitive understanding of it, that we could not say that we were both referring to the same object.

The second underlying flaw with Platonism is its lack of an answer to the question of how mathematics, so viewed, can produce theorems that are successful in making predictions about the states of affairs in physical reality. I concur with Kitcher in his 
belief that Platonism does not provide us with an explanation of why mathematics is useful.

"Mathematical truths are useful to us. But why are they so useful? An answer to this question need not be a direct consequence of a mathematical theory. However, a good theory of mathematical reality ought not to make this sensible question look like an unfathomable mystery. But that, I maintain, is what Platonism does.” (Kitcher 1984, p. 104-5)

Why should abstract objects be so important? How does the study of abstract objects improve our understanding of the behaviour and nature of physical objects? What is the connection between these abstractions and the familiar objects of our physical reality? Why should having knowledge of certain theorems that hold true in the Platonist's abstract realm have any bearing on our knowledge of what is (possibly) the case for physical things? The Platonist needs to be able to answer these questions else the success of applied mathematics will be nothing short of miraculous.

The Platonist is able to answer these questions by biting the Platonistic bullet and accepting the Platonic view of mathematics as a part of the wider picture found in Plato's theory of the forms. In this theory physical objects possess qualities because they "participate" in the idealisation of that quality which exists in Plato's heaven. Thus a picture can be said to have the property of being beautiful because it participates in the pure form of beauty, and a group of objects can be said to have the property of, for example, sixness because they participate in the pure form, or ideal, of six. Both the ideal of beauty and the ideal of six exist in heaven.

It is interesting to note that if we consider the Fregan definition of numbers, then we will find that it has a definite Platonic ring to it. Frege defined numbers to be properties held by certain classes of objects. These properties that the classes of objects have would best be described as oneness, twoness, threeness, etc. So the number twelve is the property that all dozens have in common, the property in virtue of which we label them dozens, namely their twelveness. 
If we are to accept that the connection between physical objects and ideals is as Plato described, then physical objects on Earth could then be said to conform to mathematics because of this relationship between physical objects and the pure forms. Therefore, if mathematics teaches us about the ideal world of Plato's heaven, then it will also tell us about the behaviour of physical Earthly objects as their behaviour is a reflection of what takes place in heaven. Of course, nobody is likely to support such a view today. However, it is not too far removed from a popular and appealing answer.

The popular response for Platonists in the modern era is to say that these abstract structures are the embodiment of certain structural properties of the physical universe. That the study of these abstract objects reveals certain structural properties present in the physical world, and that mathematical statements are true in virtue of these structural properties.

The question still remains, how do we know about these structural properties? If I know that a particular statement in mathematics is true, then, according to this theory, the statement is true in virtue of the structural properties of the universe, and that it is through my intuition about the structural nature of things that I have access to this fact. My claim is that surely if mathematics is about the structural properties of the universe, then it makes more sense for us to access these properties through some sort of empirical investigation carried out in reality than through pure intuition. Why should we have an intuitive grasp of these structural properties? It sounds so much more reasonable to say that we discovered them through experience.

Even if the Platonist can come up with an adequate theory to explain our epistemological connection to these abstract objects, and we hold the view that mathematics works for the world of physical objects because it describes the realm of abstract objects that embodies the structure of physical reality, the task of the Platonist is then to describe such a realm and show that it has this property.

"Problems begin to arise when the Platonist tries to produce the abstract objects which embody the structure in virtue of which arithmetic is true. His theory gives too many abstract instantiations of that structure, the von Neumann numbers, the Zermelo numbers, and so forth. ... He tries to isolate 
what is common to these various sequences of abstract objects, arriving at a view which is like that of White or Field." (Kitcher 1978, p. 131)

In Kitcher's reformulation of Benacerraf's arguments against arithmetical Platonism from "What Numbers Could Not Be", he asks us to reflect on the inconsistency of the following statements:

(1) The numerals are singular terms which refer to abstract objects.

(2) If the numerals refer to objects then there are particular objects to which they refer.

(3) There are no particular sets to which the numerals refer.

(4) There are no abstract objects distinct from sets.

If we fix our set theory as ZF, a reasonable thing to do, and then add to ZF set theoretical expressions that have the effect of defining a " 0 " and a "successor relation", and then impose the Peano postulates we will generate an arithmetic that Kitcher calls an "ersatz arithmetic". According to Benacerraf there are many such ersatz arithmetics, and associated with each arithmetic is a sequence of sets, which are its numbers, and a set theoretic relation, which is its successor relation. Therefore, even within these parameters many different arithmetics can be produced, and it would be arbitrary to claim that any one of them is the abstract realm we are looking for.

"Canons of economy and explanatory unification direct the Platonist to see his realm of Platonic entities as a universe of sets. But the notion of sets is insufficient to yield a mathematical theory which can be interpreted as describing the abstract structure of the world. ... As we have seen, purely set theoretic developments of mathematics must arbitrarily assign a special status to particular instantiations of mathematical structures." (Kitcher 1978, p. 131)

Kitcher believes that this is a fatal flaw for Platonism:

"Here then is the plight of the Platonist. He must either flout principles of economy and explanatory unification or he must opt for some arbitrary set- 
theoretic development of mathematics, which vitiates any claim that talk of abstract objects is a method of talking about abstract structure." (Kitcher 1978, p. 131).

This problem for Platonism with respect to arithmetic is similar to the problem of conventionalism with respect to geometry. Here we find that the Platonist must make an arbitrary choice between different set theoretical formulations all of which result in the same arithmetic. Later we will find conventionalists making an arbitrary choice between different metrical formulations all of which result in different geometries.

Even if the Platonist can convince us that there is an abstract realm, maybe with set theoretical foundations, that can be chosen in a non-arbitrary way, then we still need to ask why this realm has the property of being descriptive of the structural nature of reality. This, to me, is the crucial problem for Platonism, for without an answer we cannot explain the success of mathematics.

In conclusion, we find that even though Platonism is a widely held theory of mathematics we find that, under closer examination, the theory is riddled with problems. The first major problem is that with a causal theory of knowledge it is difficult to explain how we can have access to abstract entities in an epistemologically significant way, so my first criterion for a good theory of mathematics is unsatisfied. Furthermore, it is difficult to explain how these abstract objects can have any relevance to the physical world, so my fourth criterion for a good theory, that it should explain the success of mathematics, is also unsatisfied. Therefore, even though Platonism does fit with the best practice of mathematicians, and does provide an ontological theory for mathematics, it would be best to seek a more appealing alternative. 


\section{§3.3 Formalism}

The key doctrine of formalism is that mathematics is just a process of manipulating symbols on the page. So when a mathematician differentiates a function, she is merely manipulating symbols in accordance with a set of rules. There are no doubt good ways and bad ways to manipulate symbols, and mathematics aims to lay down the formal rules of how to manipulate them in the best way possible.

Formalism developed out of the logical positivist's desire to banish all metaphysical ontology of the Platonic kind. The formalist's ontological stance is that mathematical objects have no existence beyond the symbols we see and words we hear. Therefore mathematics can be said to have no subject matter and be void of factual content. Mathematics is the study of the logical relations that exist between concepts. Hence this viewpoint is also referred to as "logicism".

Hersh and Davis describe formalism as a kind of backstop position for working mathematicians. They claim that mathematicians are Platonists at heart, but will fall back to formalism when they need to explain their work in philosophical terms. This indicates an initial problem for formalism because it raises the question does formalism fit with the best practices of working mathematicians? Often mathematics, especially algebra, does seem like nothing more than manipulating symbols on a page, but I am sure that most mathematicians want to believe that there is more to it than that. If formalism is able to provide sound explanations of the other questions we are asking then maybe we should conclude that the Platonistic feelings that mathematicians have are mere illusions. However, if it turns out that we want to adopt a theory that claims that mathematical entities have no objective reality, then we should at least be able to provide an explanation as to why so many mathematicians are deluded. Can formalism do this?

Even though formalism may seem initially unattractive to a mathematician, Hempel makes a very compelling argument for it in his article "On the Nature of Mathematical Truth" which Benacerraf and Putnam describe as a "very clear and lucid exposition of logicism." (Benacerraf and Putnam 1964, p. 1) In this article 
Hempel claims that mathematics, properly conceived, is the study of logical consequences of axioms. Mathematical statements do not tell us anything about what the mathematical objects are, but that anything that satisfies the axioms of a system must also satisfy its theorems.

“On the Nature of Mathematical Truth" (Hempel 1964) deals specifically with arithmetic, algebra, and analysis, for issues concerning geometry Hempel refers the reader to his article "Geometry and the Empirical Science" (Hempel 1945). I will use both articles to form the basis of this discussion on the Formalism.

Hempel stresses the implicational nature of mathematics and describes mathematics as a collection of axiomatic systems. The proof of a theorem shows that the theorem is logically implied by the axioms, in other words, that the theorem is true provided that the postulates are true. Therefore when we state that a given theorem is true, what we are really stating is that if the axioms are true, then so too is the theorem. Therefore all mathematical truths are analytic and a priori.

This notion of mathematical truth has been dealt with in the Section 2.2 on apriorism, I only repeat it here to show that this is the kind of mathematical truth that formalism compels us to embrace. If we are to adopt formalism, then we will be burdened with all the problems an aprioristic view of mathematics entails, but it is the ontological issues that we are concerned with for the present.

To answer the question of how it is that geometry appears to make many true statements about physical reality, Hempel stresses the need for a distinction between mathematical geometry and physical geometry. In physical geometry the mathematician (or physicist) assigns meaning to the primitive geometric terms and concepts by assigning specific designata to each of the primitive terms, thereby obtaining a "semantical interpretation of a pure mathematical theory." (Hempel 1945, p. 13)

The solution to the question of the success of mathematics seems simple. All we need to do is to assign referents to the primitive terms of our axiomatic systems, then, 
provided that the axioms hold true for these referents, all of the theorems will hold true also. In reference to the postulates of Peano arithmetic, Hempel states:

"All we can assert so far is that any specific interpretation of the primitives which satisfies the five postulates - i.e., turns them into true sentences - will also satisfy all the theorems deduced from them." (Hempel 1964, p. 373)

However, in the case of geometry it is difficult to determine exactly what this designatum is. Hempel writes that the physicist connects to geometrical terms a "more or less definite physical meaning." By which he claims that points are designated to "physical points, i.e., objects of the kind illustrated by pin-points, cross hairs, etc. Similarly, the term 'straight line' refers to straight lines in the sense of physics, such as illustrated by taut strings or by the path of light rays in a homogeneous medium.” (Hempel 1945, p. 13)

Having given the primitive terms a physical meaning, all the postulates and theorems of the system can then be said to be statements of physics, and it is then meaningful to ask whether or not they are true. For example "the theorem about the sum of the angles in a triangle turns into an assertion that the sum of the angles (in a physical sense) of a figure bounded by the paths of three light rays equals two right angles." (Hempel 1945, p. 14)

"Thus, the physical interpretation transforms a given pure geometrical theory - Euclidean or non-Euclidean - into a system of physical hypothesis which, if true, might be said to constitute a theory of the structure of physical space." (Hempel 1945, p. 14)

All a mathematician can say is that if all the postulates of a theory are true in physical space, then all the theorems must also be true, since they are logically deducible from the postulates. This suggests a simple process of testing the postulates empirically. However, in the case of the geometries under discussion, this is not feasible as it is not possible, in practice, to construct a direct experimental test for the postulate of the parallels. The best scientists can do is to derive consequences from the postulates, use 
these to make predictions, and then either confirm (until further notice) or disconfirm the theory.

"While formal logic and pure mathematics do not in themselves establish any assertions about matters of empirical fact, they provide an efficient and entirely indispensable machinery for deducing, from abstract theoretical assumptions ... consequences concrete and specific enough to be accessible to direct experimental test." (Hempel 1945, p. 14)

For example, from the postulates of Euclidean, hyperbolic and elliptic geometry different conclusions about the angle sum of a triangle are deduced, and this is open for empirical testing. Hempel points out that Gauss himself actually attempted to perform such an experiment using sighting equipment (i.e., using light rays) from the tops of three mountains, the deviation from two right angles was, however, within the limits of experimental error, so Euclidean geometry was not disconfirmed, (but neither were hyperbolic or elliptic geometry).

Reichenbach also endorses the implicational nature of pure geometry and the distinction between mathematical and physical space outlined above, and he explains that physicists may use experimental methods, such as those of Gauss, to determine which geometric system should be used to describe physical space:

"Up to that time physics had assumed the axioms of geometry as the selfevident basis of its description of nature. If several kinds of geometries were regarded as mathematically equivalent, the question arose which of these geometries was applicable to physical reality. ...Mathematics shows a variety of possible forms of relations among which physics selects the real one by means of observations and experiments. Mathematics, for instance, teaches how the planets would move if the force of attraction of the sun should decrease with the second, third or nth power of distance; physics decides that the second power holds in the real world. ... After the discoveries of nonEuclidean geometries the duality of physical and possible space was recognised. Mathematics reveals the possible spaces; physics decides which among them corresponds to physical space." (Reichenbach 1958, p. 6) 
This all seems very reasonable. When we leave the primitives undefined, we are carrying out pure geometry and are only interested in which statements may be derived logically from which. However, as soon as we assign some physical definitions to the primitive terms, then we are performing physical geometry and need to test our axioms for truth by empirical means. We can deduce that Euclidean geometry yields (approximately) true statements for the physical world because when we assign the usual physical definitions to the primitives of the axiomatic system, the axioms turn into (approximately) true statements. This is the case for empirical investigations conducted in the local region of the physical world that we inhabit.

“In comparatively small areas, such as those involved in Gauss' experiment, Euclidean geometry can serve as a good approximative account of the geometrical structure.” (Hempel 1945, p. 16)

However, when we test the consequences of Euclidean geometry over the vast distances of space with straight lines defined to be rays of light, then we find that the relativistic non-Euclidean geometry is better supported by our observations. In 1919 Eddington and Cottingham conducted experiments involving the passage of light rays originating from distant stars and passing close to the sun during a solar eclipse. According to Newtonian physics (based on Euclidean geometry) the apparent location of the star should not have changed, but, according to relativistic physics (based on non-Euclidean geometry) the mass of the sun should have caused the light rays from the star to curve giving an apparent location slightly different from that given by Newtonian physics. The experiment was heralded as a success and provided evidence to accept relativistic physics over Newtonian physics, thereby accepting a nonEuclidean geometry as the geometric structure of physical space over Euclidean geometry.

"In our physical universe, the curvature of space at a given point is determined by the distribution of masses in its neighbourhood; near large masses such as the sun, space is strongly curved, while in regions of low mass-density, the structure of the universe is approximately Euclidean. The hypothesis stating the connection between the mass distribution and the curvature of space at a 
point has been approximately confirmed by astronomical observations concerning the paths of light rays in the gravitational field of the sun." (Hempel 1945, p. 16)

Therefore for space as a whole we find the physical instantiation of Euclidean geometry to be insufficient. The logical consequences of the more complicated nonEuclidean geometry of the theory of relativity, when interpreted as physical geometry, yields statements that may be empirically verified where Euclidean geometry does not.

\begin{abstract}
"Certain findings in astronomy represent good evidence in favour of a total physical theory with a non-Euclidean geometry as part $G$. According to this theory, the physical universe at large is a three-dimensional curved space of a very complex geometrical structure; it is finite in volume and yet unbounded in all directions. ... Physical space is a three-dimensional analogue to that of the surface of a sphere, or, to be more exact, to that of the closed and finite surface of a potato, whose curvature varies from point to point." (Hempel
\end{abstract} 1945 , p. 16)

Thus we may conclude that the geometric nature of physical space is locally Euclidean, but globally non-Euclidean.

This seems all very reasonable, but have we really answered the question why geometry is so successful? I find the following quote enlightening:

"Geometry thus construed is a purely formal discipline; we shall refer to it also as pure geometry. A pure geometry, then, - no matter whether it is of the Euclidean or of a non-Euclidean variety - deals with no specific subjectmatter; in particular, it asserts nothing about physical space. All its theorems are analytic and thus true with certainty precisely because they are devoid of factual content. Thus, to characterize the import of pure geometry, we might use the standard form of a movie-disclaimer: No portrayal of the characteristics of geometrical figures or of the spatial properties or relationships of actual physical bodies is intended, and any similarities 
between the primitive concepts and their customary geometrical connotations are purely coincidental.” (Hempel 1945, p. 12)

To say that the success of mathematics in making predictions in the physical world is purely coincidental is highly unsatisfactory. In fact, this is the key problem with formalism. Formalism explains how truth is established within the frameworks of mathematics, but provides no reason why mathematics has a high degree of success in making predictions about physical reality. Formalism states that we may apply physical meanings to the primitive terms to yield a physical theory which we may test empirically. However, why it is that some of these theories do in fact work is a complete mystery. If what we are looking for is a theory that does not make the success of mathematics a miracle, or a coincidence, then we need to keep looking.

Therefore I conclude that we should not adopt a formalist approach to geometry. While I can see the strength of this position, I object to it on two grounds. Firstly, I have stated as a criterion that a philosophy of mathematics should gel with the best practices of the working mathematician. While I concede that sometimes mathematics does seem like it is purely the manipulation of symbols, for the most part mathematicians go about their work with a Platonistic outlook, if this outlook is mistaken, then we should at least know why, and formalism offers no explanation. Secondly, from the criterion that a philosophy of mathematics should explain the success of mathematics in making predictions about the world, (or at least should not make it a complete mystery), if mathematics is nothing more than the manipulation of meaningless symbols, then I can see no reason to suppose that it should have any predictive power whatsoever.

I believe that Hempel's movie disclaimer analogy hints at a very interesting alternative. I believe that this analogy is closer to the mark than Hempel imagined. Movies are works of fiction, and if we consider geometries in this light, then, I believe, that we can generate a theory of mathematics that will satisfy our entire desiderata. 


\section{§3.4 Kitcherian Ontology}

Since we have decided to adopt the quasi-empirical epistemological views of Kitcher, we now need to look more closely at the theory to see what ontological commitments the theory leads us to make. Kitcher sees Benacerraf's arguments against Platonism as decisive and offers an alternative theory of mathematics that is based on idealising the operations rather than the entities. Kitcher's theory is specifically about arithmetic, so our first investigation is to decide whether or not it is a good theory of arithmetic, and then, after deciding that it is, we shall see in the next chapter if it can be applied to geometry.

One of the arguments against Platonism in the Section 3.2 was that if we are to regard mathematical objects as real abstract entities, then the canons of parsimony and theoretical unification demand that we view them as sets. However, we can use set theory to build a variety of ersatz arithmetics, all of which encapsulate the real number arithmetic that we desire. The problem is then to decide in a non-arbitrary way which is the abstract structure that forms the foundation for arithmetic. Kitcher regards this problem as an unsolvable puzzle for the Platonist, and although Kitcher does not use this problem in this way, I believe that it has explanatory value in motivating Kitcher's views on mathematics.

If we look at all the candidate ersatz arithmetics, and look for what each has in common, then we find that the key factors in the foundations of arithmetic are the successor relation and a starting point. Looking at this problem from a Platonist's perspective we are driven by ontological parsimony to locate exactly one realm of entities for the foundations of mathematics. But if we shed our Platonistic ontological commitments then why does there need to be only one abstract realm that embodies the relational structure of physical things? If we adopt a fictionalist outlook then having many arithmetics no longer carries an ontological burden, but rather has explanatory value. From the fact that there are many equivalent theories of arithmetic, surely we should suspect that any theory that contains a starting point and a successor relation will do the job. Therefore the starting point and successor relation become likely candidates for the foundations of arithmetic. 
The genius of Kitcher's thesis is to focus not on the abstract entities themselves, but on the operations that we perform on them. In light of the above paragraph a sensible starting place would be the operations of finding a starting place and determining a successor, and this is exactly what Kitcher does using as primitive the action of collecting.

"Can we rewrite sentences of arithmetic in a way which will avoid reference to abstract objects? Let us take as primitive the notions of unitary collecting, the notion of one collecting being a successor of another and the notion of collectings being matchable. (These notions are easily interpretable in terms of our crude paradigm: the first corresponds to 'making one', the second to 'adding one', while two collectings are matchable if it is possible to follow their performance with an operation correlating the objects collected). We can now reformulate first-order arithmetic.” (Kitcher 1978, p. 132)

Kitcher claims that using the operations as primitives we can reword the Peano postulates and derive an analogous arithmetic with all the usual theorems. He demonstrates this by developing what he terms Mill arithmetic, in recognition of the empirical nature of his arithmetic, and succeeds in building an arithmetic that is the same as normal arithmetic in every way except at the very foundations.

"I shall rewrite statements of first-order additive arithmetic in a first-order language, the language of Mill Arithmetic. The primitive notions to be used are those of a one-operation, of one operation being a successor to another, of an operation being an addition on other operations, and of the matchability of operations." (Kitcher 1978, p. 112)

Kitcher believes that by regarding mathematics as a science of ideal operations we can develop a theory of mathematics in which mathematics can truly be said to describe the structure of physical reality.

"I think we can do better. We can try to articulate Russell's dark utterance by thinking of the structural properties of ordinary objects as determinants of the 
ways in which we can operate upon those objects. Arithmetic can be regarded as a science of ideal operations. Its utility arises from the fact that operations we perform on ordinary objects approximate the characteristics of these ideal operations.” (Kitcher 1978, p. 132)

"Russell's dark utterance" refers to a passage where Russell hints at the belief that mathematics is the study of the structural properties of physical reality.

An important aspect of Kitcher's theory is that these primitive notions are derived from our experiences manipulating medium sized physical objects. He describes a "crude paradigm" of teaching children the meaning of sets and of addition through the action of collecting and the notion of a collection as demonstrated using blocks. It is these actions that we idealise into a theory of mathematics. Kitcher describes how the act of collecting is learned by first engaging in the physical act of gathering certain objects together, but then is extended to a mental act of collecting objects in our heads. He also describes the act of correlating, which also begins with matching up physical objects but "as we become familiar with the activity we no longer need the physical props." (Kitcher 1978, p. 111)

The crude paradigm of manipulating objects through the actions of forming collections provides a quasi-empirical foundation for mathematics and ensures that there is a tangible link between mathematics and the physical world. This is something that both Platonism and formalism lack, and it is through this connection that we can explain the utility of mathematics.

"We wanted to be able to explain the usefulness of arithmetic. My proposal provides an explanation. Arithmetic is useful because the manipulations we perform on physical objects approximately satisfy the constraints which the arithmetical axioms place on the arithmetical operations, just as Euclidean geometry is useful because the contours of physical objects approximately satisfy the constraints which the Euclidean axioms place on geometrical figures." (Kitcher 1978, p. 133) 
We need to be clearer about the nature of these ideal operations. It is clear that construing the structure of physical reality to be manifested in the operations we actually perform is inadequate. With our biological limitations, the operations we actually engage in are insufficient to build an adequate theory of mathematics. However, if we use these operations as a base and extrapolate to operations which we could perform if we were freed from our accidental physical limitations, then we can arrive at a sufficient theory of mathematics. It is useful to imagine an ideal agent, one that is not bound by our physical limitations, and to say that mathematics is the study of the actions of such an ideal agent.
"Arithmetic owes its truth not to the actual operations of actual human agents, but to the ideal operations performed by ideal agents. In other words, I construe arithmetic as an idealizing theory: the relation between arithmetic and the actual operations of human agents parallels that between the laws of ideal gases and the actual gases which exist in our world. We may personify the idealization, by thinking of arithmetic as describing the constructive output of an ideal subject, whose status as an ideal subject resides in her freedom from certain accidental limitations imposed on us." (Kitcher 1978, p. 109)

Kitcher is quite clear that we are not committing ourselves to the belief in the existence of some mysterious being with superhuman powers. "On my account of arithmetic, there is no commitment to the existence of an ideal agent or to ideal operations." (Kitcher 1978, p. 117) We envisage an ideal agent by stipulating the powers of this agent by characterizing certain principles which we observe hold true for our actual performance of these operations. This stipulation is warranted by our recognition that the ideal operations which we attribute to the ideal agent are abstracted from our actual operations by removing the accidental physical limitations of our own performances.

We may then say that mathematical statements are true in virtue of the stipulations that we have laid down. While these stipulations do correspond to our actual experiences manipulating physical objects, Kitcher claims that the way we have arrived at these stipulations through a chain of transmission that began from the experiences of those who first initiated the study of mathematics in the ancient world. 
This knowledge has then been transmitted from one society to its successor, and as individuals we receive this knowledge from contemporary society.

Kitcher gives a more definite statement of his central thesis:

"I propose that the view that mathematics describes the structure of reality should be articulated as the claim that mathematics describes the operational activity of an ideal subject. In other words, to say that mathematics is true in virtue of ideal operations is to explicate the thesis that mathematics describes the structure of the world. Obviously, the ideal subject is an idealization of ourselves, but I explicitly reject the epistemological view that we can know $a$ priori the ways in which the idealization should be made. Finally, I interpret the actual operations, for which mathematics provides an idealized description, as comprising both collective and correlative operations." (Kitcher 1978, p. 111)

Kitcher concludes:

"My solution to Benacerraf's problem motivates the mathematician's distinction between sets and functions by regarding mathematics in general as a system, like geometry, which we apply to explain our experience. To say that Euclidean geometry is (approximately) true is to avow the ability of Euclidean geometry to explain and describe, inter alia, the contours of physical objects. To say that set theory and arithmetic are (approximately) true is to vouch for the ability of set theory and arithmetic to explain and describe, inter alia, our manipulations of physical objects. If these latter theories are to serve that purpose they must differentiate correlative and collective operations. But, just as acceptance of the approximate truth of geometry entails no acceptance of the actual existence of ideal geometrical objects, so too our acceptance of the approximate truth of set theory and arithmetic does not fill our ontology with Platonic entities." (Kitcher 1978, p. 134) 
"We can abandon Platonism, and, if we do, I think we can escape the plight of the Platonist.” (Kitcher 1978, p. 135)

I believe that Kitcher has solved the problems of Platonism and formalism and arrived at a good philosophical account of arithmetic. By focusing on the operations rather than the entities he has avoided the ontological problems of Platonism, and by basing the ideal operations on the actual operations we may perform, he has explained the success of mathematics in making predictions about the states of affairs in physical reality. The question now needs to be asked, can this theory work for geometry? I believe that by using projective geometry in a way similar to Kitcher's use of Peano arithmetic, we can arrive at a Kitcherian picture of all geometry. This will be the focus of Chapter Four. 


\section{§3.5 Fictionalism}

As we have already seen, most working mathematicians have a Platonistic outlook towards the ontology of mathematics, and as we have also seen, Platonism is riddled with so many philosophical difficulties that it is not an attractive position to try to defend. As a result Hersh and Davis claim that many mathematicians will resort to a formalist position when faced with these philosophical issues and quote Dieudonne who captured this sentiment very nicely:

"On the foundations we believe in the reality of mathematics, but of course when philosophers attack us with their paradoxes we rush and hide behind formalism and say, 'Mathematics is just a combination of meaningless symbols', and then bring out Chapters 1 and 2 on set theory. Finally we are left in peace to go back to our mathematics and do it as we have always done, with the feeling each mathematician has that he is working on something real. This sensation is probably an illusion, but is very convenient. That is Bourbaki's attitude towards foundations." (Davis and Hersh 1981, p. 321)

My claim is that instead of running and hiding behind formalism to defend her Platonistic outlook, the mathematician has a more attractive alternative: fictionalism. Fictionalism offers so many more advantages than the positions considered so far, it has a clean and parsimonious ontology, it gives a clear explanation of the epistemological issues related to mathematical knowledge, it actually does fit with the feelings of the working mathematician and explains her Platonistic instincts, and finally, when developed out of Kitcher's quasi-empirical framework outlined above, explains the utility of mathematics. In other words, this theory has everything we are looking for.

In Gideon Rosen's article “Modal Fictionalism” (Rosen 1990) he presents a theory of possible worlds known as "fictionalism". To explain the central idea of fictionalism he asks us to consider the statement:

(1) There is a brilliant detective at $221 \mathrm{~b}$ Baker Street. 
This statement, when taken as a straightforward existential claim, must be considered false. But according to the fictionalist what we mean by (1) is in fact the following:

(2) In the Holmes stories, there is a brilliant detective at 221b Baker Street.

This is a claim that is true. Rosen calls an operator of the form "In the fiction $F, \ldots$ " a story prefix. Using the story prefix we may make true claims about what is the case within fictions without committing ourselves to the belief that the claims are actually true.

"We may generalize further by noting that the story mentioned in a story prefix need not be a literary fiction, nor for that matter, any sort of fiction in the usual sense. ... In general, the fiction mentioned in a story prefix can be any representation whatsoever: a story, a scientific theory, or a metaphysical speculation” (Rosen 1990, p. 331)

Of course, the fiction mentioned in the story prefix that we are interested in will be a mathematical theory. In particular we will be considering the various geometries as fictions. I am going to assert that any geometric statement must be prefixed by what Rosen calls a story prefix of the form "In the geometric theory $G, \ldots$ " so that we may know which geometry we are talking about. Of course, if it is clear from context, this prefix may be omitted, but it is implied.

Rosen goes on to develop a view of fictionalism with regard to possible worlds pointing out its strengths and weaknesses. The view is built on the shoulders of David Lewis's modal realism as presented in On the Plurality of Worlds (Lewis 1986). I am not going to concern myself with defending fictionalism with respect to possible worlds; I only want to note that it is a well respected theory of modality and that there are many reasons why we might want to adopt it. My principle concern is to show how such a view may be applied to geometry, and to consider the advantages and 
disadvantages of doing so, ultimately concluding that the good outweigh the bad, and that it is an appealing theory to adopt.

My first step is to show the compatibility of fictionalism with regard to geometry. Contrast (1) and (2) with the following:

(3) The angle sum of any triangle is two right angles.

(4) In Euclidean geometry, the angle sum of any triangle is two right angles.

(3) is a claim that, if we accept relativity theory, we must consider, strictly speaking, to be false, yet (4) is clearly true. The parallels to (1) and (2) should be obvious. This appeals to the distinction between physical geometry and mathematical geometry drawn by both Hempel and Reichenbach. Statements such as (3) are taken to be about physical geometry and are either true or false of the world, whereas statements such as (4) are about mathematical geometry and are either true or false within the geometry in question.

Crittenden alerts us to several distinct kinds of sentences about fictional objects, giving the following as examples:

(5) Sherlock Holmes smokes a pipe.

(6) Holmes was created by Arthur Conan Doyle.

The first statement is made about the states of affairs within the fictional world, and so Crittenden calls sentences of this kind "inside" statements. The second is a statement about empirical reality, therefore the story prefix does not apply; he calls sentences of this kind "outside" statements. (Crittenden 1991, p. 94-5) This is clearly the same kind of distinction made by the positivists in what they called "internal" and "external" statements. This is an important distinction to draw. In mathematics in order to determine the truth or falsity of inside, or internal, statements such as (3) we need to look inside the mathematical theory in question. Meta-theoretical statements 
such as Gödel's incompleteness theorem fall into the second category as they are statements about mathematical theories.

Not all statements referring to fictions and fictional entities fall neatly into these two categories, however. Consider the statement "I am taller than Frodo Baggins." This requires a comparison between a fictional character and a real person. We will ignore these difficulties as they do not seem relevant to the issues concerning geometry.

To ascertain the truth or falsity of an inside statement Crittenden claims that all we need to do is read the relevant work of fiction. To ascertain the truth of a mathematical inside question then, by analogy, all we need to do is examine the postulates of the system and see if we can derive the statement as a theorem.

Therefore a statement can be said to be true in a geometry if and only if it is theorem of that geometry.

This view also highlights the implicational nature of geometry. If we make the claim that "in the geometry $G, A$ " then what we mean is that $A$ is a theorem of $G$, or, $A$ is a logical consequence of the axioms of $G$. This conforms to Hempel's description of the nature of mathematical statements discussed in Section 2.2, where we saw that Hempel described all theorems of geometry as conditional statements that state that the theorem is implied by the conjunction of the postulates. This kind of "if-thenism" does not necessarily lead to the kind of apriorism that Hempel envisaged. It is also quite at home in the fictionalist's view of mathematics.

We may say that a theorem is true of a given geometry, but is only possibly true of physical reality. Thus, we may describe the fictionalist's account of mathematics as modal. i.e., the study of possibilia. Each geometry may be considered as a possible world and a theorem that is true in one geometry would be said to be true in a possible world, and therefore possibly true (of reality).

Now that we have a rough outline of what the fictionalist's stance is, we may explore the benefits of holding such a position. Rosen cites Chihara's theory of fictionalism in mathematics as an inspiration for his fictionalism of possible worlds. Just as Rosen aims to use fictionalism to get all the benefits of realism with regard to possible worlds without the ontological costs, Chirara aims to get all the benefits of 
mathematical realism (Platonism) without its ontological baggage. The benefits of Platonism are outlined by Chihara as follows:
"Initially, the philosophical advantages of espousing Mathematical Realism appear to be quite impressive. First of all 'genuine mathematics', as is actually practiced by mathematicians and not some philosophical version of it, can be understood to be a straightforward theory about mathematical objects. For example, it can be plausibly maintained that set theory, as it is taught in mathematics departments throughout the world, is a theory about ...SETS. Since the Mathematical Realist believes that mathematical objects truly exist, she can take the axioms of set theory to be just truths about sets. Secondly, the logical form of the statements of mathematics can be taken at face value, so that the semantics of the language used can be regarded as the standardly referential Tarskian one.” (Chihara 1998, p. 291)

So, as we have already seen, one of the advantages of Platonism is that it explains what it is that makes mathematical statements true in the standard Tarskian way. Does fictionalism also have this advantage? If we believe that statements about Sherlock Holmes, such as (1) refer, and that mathematical statements are of the same kind as such statements, then we may also claim that mathematical statements refer. Therefore we may say that set theory is about sets in the same way that Arthur Conan Doyle's novels are about Sherlock Holmes. Thus statements of set theory may be said to be true of false about the world of sets in the same way that statements about Holmes are true or false of the fictional world created in Doyle's novels. I do not wish to discuss the finer details of reference in fiction except to say that we have a highly developed and reputable theory in which reference to fictional objects may be carried out and truth may be derived in the standard Tarskian way. If we accept that mathematical theories are similar in kind to literary fictions, then we may also hold that mathematical statements refer in the standard way and our causal theory of knowledge may be preserved without postulating the existence of abstract entities in the Platonic way, and without facing the problems that Platonists have in finding a causal link between the mathematician and the entities about which she claims to have knowledge of. 
Another key benefit of Platonism, which fictionalism also claims, is that it allows science to work unimpeded and that it conforms to the best practices of working mathematicians. Recall the arguments from Maddy in Section 3.2 above, where Maddy claimed that the existence of mathematical entities is presupposed by science, and that science is our best source of knowledge, therefore, on the grounds of indispensability, we should accept the existence of said entities.

Again we find that with fictionalism we may have this benefit of Platonism without incurring the ontological costs. We do not need to believe that abstract mathematical objects actually exist to gain access to the benefits of science, all we need to do is act as if they exist. This is precisely what the fictionalist does. The fictionalist says that the objects of mathematics are just fictional objects and that we can derive certain facts about them. These facts, of course, are true in the particular fiction. Scientists can then theorise as if mathematical fictions are true, and will derive the standard forms of science as we know it. It is an important consequence that scientific theories will also be considered fictions, but this is exactly what I consider science to be.

This also shows that the mathematician is not deluded when she claims that she feels as if she is working with actual objects. This view states that she actually is working as if she is dealing with actual objects, and that this is what mathematicians do. This fits with the quote from Dieudonne above, who claimed that the sensation of dealing with actual objects is probably an illusion, but is very convenient.

It is a feature of modern geometry that there is a plurality of geometric systems. This poses a problem if we wanted to claim that any one geometry is true a priori. However, within fictionalism it is to be expected that there will be different geometries, and that some statements will be true in one geometry but false in another. So we have further evidence of fictionalism fitting with the actual practice of mathematicians.

Of course the main advantage of fictionalism is as an ontological doctrine. We state that mathematical objects are fictional entities, not actually existing abstract entities. On this view works of fictions are things that really do exist, they are things we want to have in our ontological list, but the objects within them do not really exist. In the 
same way mathematical theories do exist, but the entities within them do not. By considering mathematical theories to be the same kind of entities as other fictions, which we already take to exist, we are not increasing the number of different kinds of entities in our ontology, and we may consider the ontological issue closed in a neat and parsimonious manner.

Therefore we can see that fictionalism has numerous advantages over its rivals. It allows mathematical statements to refer and therefore be true in the standard Tarskian way, it settles the ontological problem of abstract entities in a satisfactory way, it makes sense of the existence of a plurality of geometries, and it fits with the best practice of working mathematicians explaining their inclination towards Platonism. The remaining problem is to answer how fictionalism can explain the success of mathematics in the physical world, and to make clear how we can come to have knowledge of what is the case in these fictional worlds.

Putnam rejects fictionalism claiming that "realism is the only philosophy that doesn't make the success of the science a miracle." (Putnam 1986, p. 60) He describes a realist as someone that (1) holds that the sentences of a theory are true or false and (2) holds that what makes these sentences true is something "external". It should be clear that this view of realism does not commit the realist to Platonism, and that the quasiempirical view outlined above fits into Putnam's category as a realist theory. My task is now to show that we can develop a cohesive theory of quasi-empirical-fictionalism that has all the advantages of both fictionalism and realism. I believe that we can use Kitcher's quasi-empiricism as a foundation to develop a fictionalist view of mathematics that explains mathematics' success and utility.

It is not a big step to take Kitcher's view of mathematics and develop a fictionalistic theory out of it. Kitcher himself suggests how to take the first step towards this by saying of his theory that "one can naturally think of an idealizing theory as describing a close possible world, one which is similar to the actual world but has the advantage of lending itself to simpler description." (Kitcher 1984, p. 120) Therefore, if we take our idealised mathematical theories to be descriptive of possible worlds, and if we take fictionalism to be our theory of the nature of possible worlds, we will have a fictionalist outlook for our mathematical theories. 
The kinds of possible worlds that mathematicians, and other scientists, consider may be thought of as close possible worlds because, if we have developed our theories in accordance to Kitcher's quasi-empiracal method, they are based on the operations that we may actually perform in the real world, but with only accidental and complicating factors removed. When we consider the operations that an ideal agent may perform, we idealise the operations that we ourselves may perform if our accidental physical limitations were removed. We also ignore many aspects of reality that we deem to be irrelevant to the operations that we are concerned with. We then consider a setting in which the ideal agent may perform these operations as a possible world. If it turns out that the results of considering operations within a given possible world yields results that have a high correlation to physical reality, then we may deem the possible world to be a close possible world. Therefore, by adopting the view that mathematical theories describe possible worlds, we may interpret the claim that the sentences of mathematics that we usually accept are approximately true of the physical operations we perform as the claim that "there is a close possible world at which those sentences are true of our physical manipulations." (Kitcher 1984, p. 136 footnote 3)

Even though Kitcher does not fully embrace this theory he does makes use of the notion of possible worlds to defend against "an apparently devastating objection," the objection to the assumption in his postulate stating that the agent may continue to add one forever, when quite clearly no human agent is able to do so.

"I claim that this point is unworrying. Arithmetic may or may not be true of the physical manipulations which we actually perform. However, there are possible worlds at which arithmetic is true of our physical collectings and we can legitimately regard our own world as an approximation to such ideal worlds." (Kitcher 1984, p. 132-3)

The reason that Kitcher does not embrace this theory is that he believes that this picture creates "epistemological worries". One problem is conceiving of how we can have knowledge about what goes on, and about what conditions are like, in the other worlds, and secondly, how does knowledge of states of affairs in other worlds give us knowledge about our own world? Kitcher believes that "however we conceive of 
possible worlds, they must be regarded as entities to which we have access via perception and inductive generalization." (Kitcher 1984, p. 121) Thus we can see that Kitcher is concerned that a theory that postulates the existence of abstract entities such as possible worlds will be prone to the same problems as Platonism, and is therefore reluctant to use such a theory.

Hinckfuss also points out this issue for fictionalism claiming that for the fictional worlds of mathematics to yield knowledge about physical reality, the fictional worlds and reality must be linked in an epistemologically relevant way.

\footnotetext{
"Incidentally, it would not be sufficient to indicate a possible mathematical model for such a space. For if the proposed surfaces, lines, and points were not to fall foul of the relationist's tendencies towards ontological reduction, the model would have to be related to physical theory in a way that was epistemologically relevant. What must be done, no matter how meaningful or true statements about such points may be, is to show how it would be possible for the existence of these entities to affect our knowledge of the world." (Hinckfuss 1975, p. 49)
}

If we can clear up any epistemological worries about how we can come to have knowledge about the states of affairs in possible worlds and how these states of affairs can have any bearing on the physical world, then we will be free to view the idealisation of our operational activities as described by Kitcher as descriptive of the operations which may be performed by ideal agents in a possible world. Both questions are answered by the fact that these idealised worlds are generated by the operations that we actually perform. We know about these worlds because we know about the operations upon which they are based. Furthermore, because the operations on which these worlds are built are the operations which we may actually perform in reality, the things which are true in these worlds have a correspondence to what is true in reality.

If we are to regard mathematical theories as idealisations of the operations that we actually perform, then we may draw an analogy to literary fictions that are based on true stories. Recall Hempel's analogy to the movie disclaimer that any relation 
between mathematics and the physical world is "purely coincidental", (Hempel 1945, p. 12) this may now be replaced by an analogy of the standard movie disclaimer for stories that are based on fact, but instead of some events being changed for dramatic purposes, some outcomes may differ from reality for the purposes of simplification. It should be clear that some fictions, namely those which are based on fact, do give us knowledge of reality, though often in a simplified way. The same is true of mathematical theories that have their foundations in empirical investigations. Euclidean geometry yields results that are approximately true because the operations of this world are idealisations of the operations that we may actually perform.

Kitcher states that if we are prepared to accept possible worlds as epistemologically unproblematic, as I am claiming, then "there is a straightforward way in which to develop the account of arithmetic which I have proposed." He calls any world which has a stock of operations which satisfy the axioms of Mill Arithmetic M-worlds. "Mill Arithmetic is applicable to our world not because our world is an M-world whose physical operations are our physical operations of segregation, but because, if accidental, complicating factors were removed, our world would be such a world." (Kitcher 1984, p. 121) Similarly, Euclidean geometry is applicable to physical reality not because reality is Euclidean, but because if complicating factors are removed then reality would be Euclidean. Alternatively, we may remove the complicating factors in a different way and generate a non-Euclidean geometry about which the same can be said.

It also appears to me that the way in which we obtain knowledge of fiction is similar to the picture Kitcher draws for how we obtain knowledge of mathematics. In Kitcher's theory contemporary individuals gain mathematical knowledge largely from the authorities of the community, in much the same way that people gain knowledge of fiction from the story telling community. In modern society this would be from the film and publishing industries. The authorities of one society gain mathematical knowledge from previous societies in much the same way that stories are handed down through the ages.

If we are to accept that we may obtain knowledge about literary fictions in an unproblematic way, and that mathematical theories are similar in kind to literary 
fictions, then we must conclude that we may obtain knowledge of mathematics in an unproblematic way also. I know certain things about the states of affairs in mathematical worlds in a similar way to knowing about the states of affairs in Middle Earth, such as that Frodo is Bilbo's nephew. This also frees us to be able to refer to our ideal agent in an unproblematic way. Kitcher explicitly stated that the ideal agent does not actually exist, and if we adopt fictionalism, then we may view the ideal agent as a similar kind of entity as a fictional character.

The analogy between literary fictions and mathematical theories as developed using a Kitcherian approach to idealising operations is not as strong as that between Kitcherian mathematics and role-playing games. In a role-playing game the nature of the fictional world generated by that game is largely determined by the set of actions that a character of the game is permitted to undertake. In much the same way the possible world generated by a mathematical theory is determined by the operations an ideal agent is deemed able to undertake.

In conclusion, my claim is that we may naturally consider mathematical theories to be theories about possible worlds, and that a very good way to describe the nature of a possible world is fictionalism. Therefore we can think of our idealised mathematical theories as describing fictions. Thus, for each mathematical theory, or system of axioms, we may imagine an ideal agent that can perform certain operations. These are our primitive operations, which are idealisations of a crude physical paradigm such as gathering blocks together. We then set certain parameters that stipulate more precisely the operations that the ideal agent may perform. Having done this the fictionalism element of our theory claims that we have defined a class of fictional worlds, i.e. the worlds in which an ideal agent may perform the stated operations within the stated parameters. Mathematicians then set about studying exactly what the ideal agent can and cannot do in all such worlds. 


\section{§3.6 Conclusion}

Even though most mathematicians conceive of mathematics in a Platonistic light, Platonism needed to be rejected on the grounds that we could not find a suitable link between the mathematician, the mathematical object, and physical reality. An epistemologically significant link is required between the knower and the object about which things are known, and, given the somewhat ethereal nature of Platonic entities, it is difficult to see how this link can be formed. Furthermore, it is difficult to see how knowledge of these Platonic entities can give us knowledge that is useful in making predictions about states of affairs in physical reality.

The formalist presents a strong picture with no ontological commitments whatever. However, the formalist can give no explanation as to why mathematical theories are successful in making predictions about physical reality, only that we can apply physical interpretations to our mathematical theories and then test to see if they are successful in yielding empirically accurate statements.

Kitcher bypassed the problems of Platonism by focusing on the operations rather than the entities and developed his quasi-empirical approach to mathematics by defining mathematics to be the study of the idealisations of the operations that we actually perform. By taking the focus off the entities and putting it onto the operations, we have avoided the ontological problems of Platonism, and by basing the operations on the operations that we actually perform, we have created that vital link between mathematics and physical reality that is required to explain the utility of mathematics.

We have then found that by using Kitcherian quasi-empiricism as a foundation, we may build a fictionalist theory of mathematics that has every feature we desire. We have a clean ontology as we do not consider fictional objects to actually exist, yet, by adopting a theory that explains reference to fictional entities, we have a means of referring to mathematical entities and of gaining knowledge about them. We also explain the mathematicians' propensity for Platonism because, under this interpretation, mathematicians do behave as if their mathematical entities were real. Finally, the success of mathematics is explained by the link to physical reality given 
in the Kitcherian notion of building our possible worlds out of the idealisation of the physical operations that we actually perform. This gives us, as an overall theory, a kind of quasi-empirical fictionalist approach to mathematics. 
Chapter 4. Geometry 


\section{\$4.1 Introduction}

In this chapter I will develop geometry in a way that parallels Kitcher's development of arithmetic outlined above. The main ontological objective is to focus on the operations rather than the entities, hence the label "operational geometry". The entities of mathematical geometry, lines, points, circles etc, I will claim do not exist. They are fictional objects and do not exist in the same way that Frodo Baggins does not exist. Our ontology is therefore cleared of the mathematical entities that clutter the Platonic world. What mathematics is concerned with are the operations that we may perform, and the idealisation of those operations. It is from this perspective that I will build our theory.

Just as Kitcher found the necessary foundations to build a theory of arithmetic in the Peano postulates, I have found the basic foundations to build geometry in the postulates of projective geometry. The keystone position held by projective geometry in the study of the foundations of geometry was brought to the attention of the academic world by Klein and Cayley who placed projective geometry as the basis for all geometries, both Euclidean and non-Euclidean. Russell, when studying these developments came to the conclusion that it was projective geometry, and not Euclidean geometry, that provided the "a priori properties of any form of externality." (Kline, forward to Russell 1956) Although my conclusion is strikingly different from Russell's, I do share the belief that projective geometry ought to play a central role in forming the foundations of geometry.

In the first section we will be developing a Kitcherian style of geometry based on the programme of von Staudt, who is credited as the parent of modern projective geometry. In 1847 von Staudt, in his Geometrie der Lage, insisted that projective geometry should be built synthetically, i.e., on a foundation in which metric ideas play no part. Thus in Section 4.2 we will be developing a purely synthetic form of geometry based entirely on operations.

Having found a synthetic approach to geometry that is based on the operations of drawing lines and performing transformations, in the Section 4.3 we will proceed on 
the analytical side following the Erlanger programme of Klein. We will determine a system of linear algebra that models our synthetic projective geometry, and see how Klein has shown that by using projective geometry as the foundation, and considering transformations as the primitive operations, a very Kitcherian approach, we may define all geometries as sub-geometries of projective geometry.

The significance of the Erlanger programme is that it shows that if we can develop projective geometry as a quasi-empirical science based on operations, and if we can define transformations as operations, then all geometries become the idealisations of operations also.

A legitimate question to ask is that if the analytical method is so powerful and convenient, why do we need the synthetic foundation at all? The solution is that it provides an epistemologically significant link to empirical reality that does away with the need to postulate abstract entities of the Platonic kind without claiming that the sentences have no meaning.

Finally, having developed a comprehensive theory of geometry from a Kitcherian quasi-empirical foundation, we will consider the problem of conventionalism, probably the most significant problem within the philosophy of geometry. We will consider how the problem is presented in the work of Poincare and Reichenbach. We will then look at Nerlich's alternative to conventionalism, and discover that following Nerlich's alternative we find ourselves once again drawn to the conclusion that the best way to conceive of geometry is quasi-empirical fictionalism. 


\section{$\S 4.2$ Operational Geometry}

In this section I will develop a theory of projective geometry that is analogous to Kitcher's Mill arithmetic. This approach will be based on drawing lines as the primitive operation and will follow the synthetic approach to projective geometry characteristic of the work of von Staudt. We will develop projective geometry to the point where we will have a system of coordinates for the real projective plane. The aim is to show that we can develop this system of geometry in a Kitcherian manner with a quasi-empirical foundation. We will then submit that this geometry may fruitfully be viewed as a fictional world.

Our first operation is drawing a straight line. A straight edge, a taut piece of string or a ray of light can be considered to be the paradigmatic methods to construct a straight line. We will follow the standard notational practices and denote lines with lower case letters $a, b, c, \ldots$ Just as Kitcher considers a child moving blocks around on the floor when first coming to grips with the concepts of arithmetic, we may consider a child with a straight edge learning to draw patterns with straight lines as forming the early foundations of the study of geometry. We learn very early on how to rule a straight line and this forms the basis of our concept of what a straight line is. When considering lines in three dimensional space the taught piece of string or beam of light will form the basic physical paradigm, but for this section we will only be considering plane geometry, hence the straight edge paradigm will suffice for now.

William Clifford in "The Postulates of the Science of Space" (Clifford 1956) claims that Euclid's definition of a line being "breadthless length" is not some abstract ideal of the Platonic kind, but an empirical claim. Clifford defines surfaces, lines and points as boundaries. He asks us to consider a glass of water, the boundary where the water meets the air may be considered a surface. This surface has no thickness for it is neither air nor water. If we consider where this surface meets the glass we will find a line, which, for the same reasons, has no breadth. (Clifford 1956, p. 555) Applying this theory to our physical paradigm, we may wish to consider the line that we draw when using a pencil to be the boundary between the graphite covered paper and the virgin paper. This will give us a line with no breadth. This is in sharp contrast to Mill 
who, as we saw in Section 2.3, claimed that "there exist no points without magnitude; no lines without breadth, nor perfectly straight." (Mill 1959, p. 145) Even though Clifford's definition of a line may account for its breadthlessness, it certainly will not account for its straightness. However, as we are creating an idealisation of our physical operations, I prefer to consider a line to be the limiting case of a sequence of narrower and narrower lines, and in any case, it is the action of creating a line that is significant, and not the line itself.

After we have accepted forming a line as a primitive operation, it is not a big step to extend this operation to continually producing a line, and have Euclid's second axiom. The physical paradigm would be lining up a straight edge along a line, and then continuing the line. We, with our physical limitations, can only produce a line so far, but the ideal mathematician may continue to produce the line indefinitely (as Euclid's axiom requires).

Once we are happy about constructing lines and continually producing them in our physical paradigm, we will soon observe that some (and I will later claim all) of our lines intersect. Thus we arrive at the primitive concept of intersection. We may then define a point to be the intersection of two lines, saying that if one line intersects another line, then we call this intersection a point, and say of both lines that they pass through this point. Again, following standard practice, we will denote these intersections, or points, with upper case letters $A, B, C, \ldots$. This fits nicely with Clifford's definition of a point, "The boundaries of a part of a line are points" (Clifford 1956, p. 555) because the intersection of two lines divides each into two parts.

Next, we need to make clear the primitive notion of incidence, that of a point lying on a line and a line going through a point (and a line being on a plane). This relation needs to be made precise. Firstly, from our definition of a point it is obvious that all points must lie on at least two lines and that lines go through points.

In our physical paradigm of drawing lines we find it a commonplace for more than two lines to intersect at the same point. This phenomenon is captured very elegantly by the set theoretical approach as it is represented by a single point being an element 
of more than two lines (which are taken to be nothing more than sets of points). In order to idealise this physical feature of line drawing we should first observe that some intersections (points) are distinct and some are not. The notion of two intersections being distinct needs to be accepted as primitive. We then say that for any three lines, $a, b, c$, if the intersection of $a$ and $b$ and the intersection of $a$ and $c$ are not distinct, then the intersection of $b$ and $c$ is not distinct from either of the first two intersections, and we may say that $a, b$, and c concur. Or, to put it another way, three lines concur if and only if the intersection of any two pairs of lines are not distinct. This definition may be generalised to any number of lines by saying that given a collection of concurrent lines, if another line concurs with any pair of lines in this collection, then it concurs with all of them. The temptation is to say that they all intersect at the same point, but this seems counter to the central theme of our development, though it may be adopted as a convenient abbreviation.

Just as it is a commonplace for two lines to pass through one point, dually, it is also common practice when drawing lines for one line to be drawn joining two distinct points. That this is always possible was given by Euclid as his first axiom. We will also be stating this as an axiom, but for the meantime we only need consider the idea that it is sometimes possible. If a line is drawn such that it concurs with two distinct intersections (points) then we shall say that the line joins the points. If it is possible to construct a line that joins three (or more) distinct intersections (points), then we shall say that the three (or more) points colline.

We may define pencil of lines to mean the collection of all lines that pass through a particular point. Dually, we may use the term series of points to mean the collection of all points of intersection on a given line.

Considering lines to be prior to points is a major break from the standard approach to the foundations of geometry. The standard approach is to begin with an undefined set of objects called points. Then introduce a collection of undefined subsets of points called lines. Then define incidence by stating that if a point is an element of a line, then we will say that it is on the line, and if a point is an element of two lines, then these lines intersect at that point. 
I initially considered having locating a point as a primitive operation similar to Kitcher's unitary collecting, but the parallels were not strong, and it seemed that this implicitly presupposed the existence of such a thing as a point prior to performing the operation. The way I have decided to proceed does not make any existential claims about pre-existing entities; we construct points by forming two intersecting lines. Quite literally, "x" marks the spot.

From these three primitive notions, forming a line, continually producing a line, and intersection (leading to the more general notion of incidence) we may develop a very elegant geometry. Just as Kitcher embodied the Peano axioms within his system to form Mill arithmetic, we may impose the following axioms on our ability to perform our primitive operations, or rather on the ideal agent's ability to perform the operations, and derive projective geometry. We therefore stipulate the following:

P1) Given any two lines, we may locate exactly one intersection of these two lines.

P2) Given any two intersections (points), we may construct exactly one line that passes through both of these two points.

P3) Through any intersection of two lines, we may construct a third, distinct, line that also passes through the intersection. (This guarantees that each point is incident with at least three lines).

P4) Given any line, we may construct two more lines, the intersection of which is not on that line.

The first three axioms stipulate that an (ideal) agent may always perform the basic operations outlined above. The fourth axiom is a non-triviality clause that guarantees that we are working on a plane, for without it we could have a single line as a geometry, and then we would not be able to do any interesting geometry at all. The usual form this axiom takes is as an existential statement claiming the existence of a line and a point that is not it. (Tuller1967, p. 35) Another, equivalent, approach is found in Redei's account of Klein's projective geometry where he asserts the existence of a triangle as the non-triviality clause (Redei 1968, p. 103). Our formulation of the axioms makes no such existential claims, but asserts that we may perform operations that will result in the construction of said line and point, or said triangle. 
The fourth axiom, together with the third, guarantees that we are able to construct three concurrent lines that are not concurrent with a given line. Or, put another way, given any line, we may construct a pencil of lines that do not include it. This axiom is required for us to perform the constructions required later to coordinatise the projective plane.

Notice that the axioms, as stated above, dictate what an ideal agent may do, and do not make any existential claims about the existence of points or lines as abstract entities.

We may prove as a theorem that every line is incident with at least three points. This, which is often given as an axiom, follows naturally from P4, P3 and P1, in that order.

The system that we have developed so far is robust enough to satisfy any mathematician's definition of a projective geometry, and we may develop some fairly interesting results from it.

One result that should be noticed immediately is the lack of parallels. If every pair of lines met axiomatically, then there can be no parallel lines. This raises an interesting question, if we are to construct a pair of lines that would be deemed parallel in the Euclidean sense, i.e., we draw a pair of lines and a transversal line such that the interior angles on one side of the transversal sum to two right angles, then these two lines, when continually produced, must meet. But given that the lines must meet exactly once, on which side of the transversal do they meet? One conclusion would be that if we continually produce the lines in both directions that we will arrive at the one intersection from both directions, and this raises the question of whether continually producing a line in one direction will result in you arriving back at your starting point from behind, something which, in the light of Clifford's comments in "The Postulates of the Science of Space", were he stated that "in fact, I do not mind confessing that I personally have often found relief from the dreary infinities of homaloidal space in the consoling hope that, after all, this other may be the true state of things." (Clifford 1956, p. 567) may not be an undesirable outcome. The projective 
solution, however, is that we simply don't have a notion of sidedness, and so the question does not make sense.

The Principle of Duality states that given any theorem of projective geometry, the statement obtained by replacing the word point with line, lie on with pass through, join with intersect and concurrent with collinear will also be a theorem of projective geometry.

Projective geometry has attracted high praise in terms of its aesthetic value, and the principle of duality is part of this aesthetic appeal. Coxeter describes projective geometry as "a very beautiful and intricate collection of propositions." (Coxeter 1969), Nerlich describes the subject as "a thing of beauty in itself." (Nerlich 1976, p. 63 ), and Kline concludes that "the science born of art proved to be an art." (Kline 1956, p. 641) But for our purposes the principle of duality is philosophically significant. Despite having derived geometry using lines as the foundation of geometry prior to deriving points as the intersections of lines, the principle of duality frees us up to talk about points and lines giving both equal status.

We may also generate finite projective planes, the smallest of which is the finite projective plane of order 2 which has 7 points, 7 lines, 3 points on each line and 3 lines on each point. This geometry may be represented by a complete quadrilateral (see below) with its four vertices and three diagonal points forming the points and the intersection of its diagonal points form the seventh line.

We define a complete quadrangle to be a shape with four vertices, of which no three colline. A complete quadrangle has six sides and the three intersections of opposite sides are called diagonal points (figure 1). The dual figure is a complete quadrilateral, which has four sides, no three of which concur, and six vertices (figure 2). 


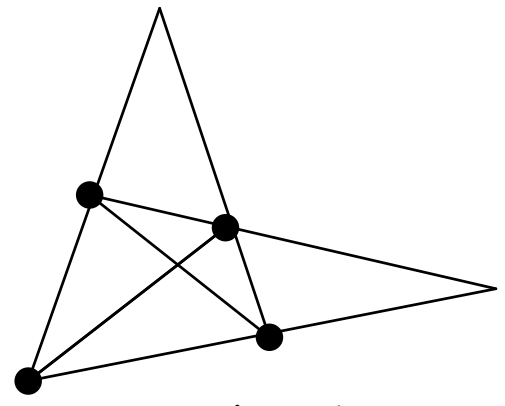

Figure 1

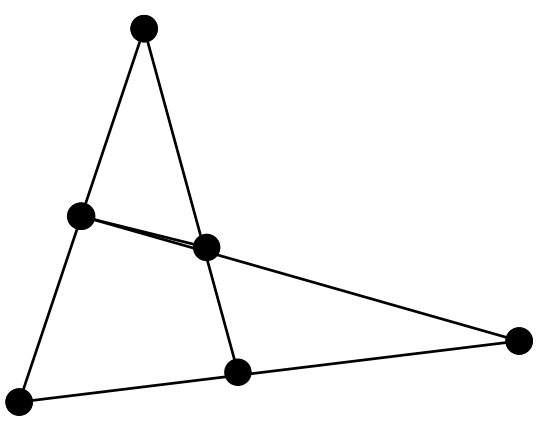

Figure 2

The finite projective plane of order two may be intellectually interesting, and does have applications in experiment design, but is counter to our empirical foundations and therefore needs to be excluded from our geometry if we are to develop a theory that is consistent with our experiences drawing lines in physical reality. Therefore we will impose Fano's axiom to rid our geometry of these anomalies.

P5) Fano's Axiom: It is impossible to draw a straight line through the diagonal points of a complete quadrangle. This ensures that the three diagonal points of a complete quadrangle are never collinear.

The empirical investigations that led to the development of projective geometry involved (as will be detailed below) projecting geometric configurations from one plane to another from the perspective of a single point located at the observer's eye. From these investigations it was discovered that certain properties of triangles and hexagons always hold regardless of how they are projected. The two most significant projective invariants to be discovered through empirical investigations are the theorem of Pappus and the theorem of Desargues.

We will want to introduce one or other of these theorems as an axiom in order for us to be able to develop a system of coordinates for the projective plane, for it is very difficult, though not impossible, to develop a system of coordinates without either. Also, it may be demonstrated that these theorems may not be derived from our axioms so far by the development of non-Desarguesian projective planes. (Robinson 1959, pp. 126-8) Tuller introduces the theorem of Desargues as an axiom, while Coxeter chooses the theorem of Pappus. I will argue for the theorem of Pappus for two 
reasons, one, when homogeneous coordinates are introduced they may be taken from any field if the theorem of Pappus is assumed to hold, but only from a division ring if Desargues and not Pappus is assumed (Interestingly this means that the theorem of Pappus implies that the commutative law of multiplication holds). Secondly, Pappus' theorem is the stronger theorem and implies Desargues' theorem, so we get two theorems for the price of one.

P6) Theorem of Pappus: If six lines are drawn such that lines may be drawn through their alternate intersections, then a line may be drawn through the intersections of the pairs of opposite sides. This implies that if the six vertices of a hexagon lie alternately on two lines, then the three points of intersection of pairs of opposite sides colline.

A diagram is useful to visualise the theorem:

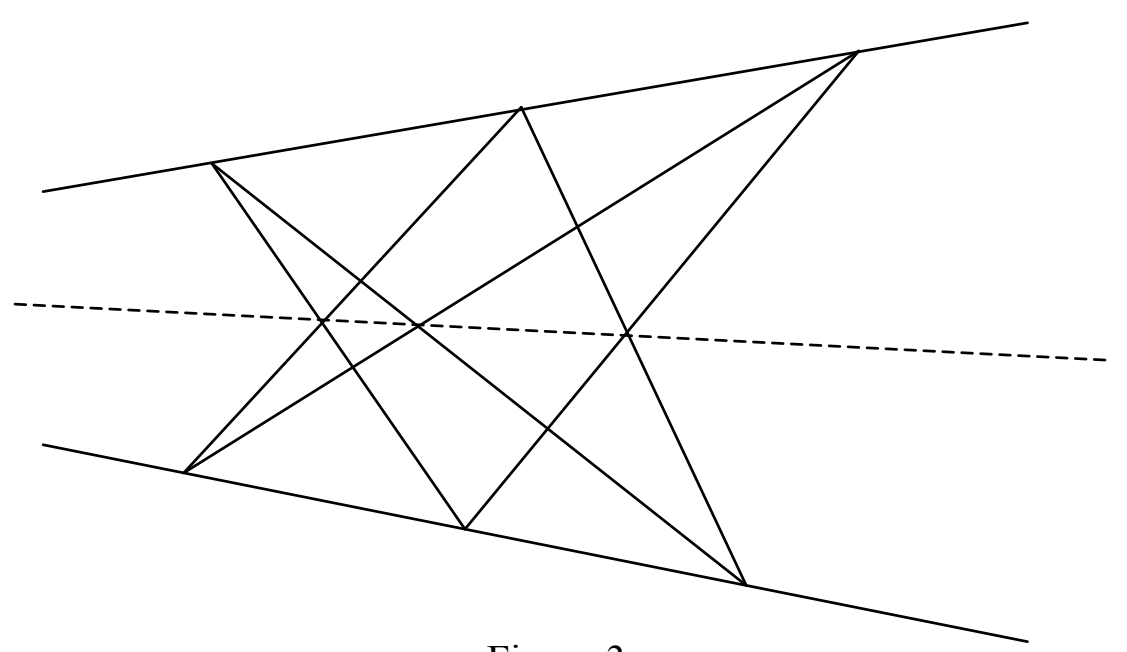

Figure 3

Before stating the theorem of Desargues, two definitions are needed: Firstly we will say that two triangles are perspective from a point just in case their three pairs of corresponding vertices are joined by concurrent lines (figure 4). Secondly, that two triangles are perspective from a line just in case the intersections of the three pairs of corresponding sides colline (figure 5). 


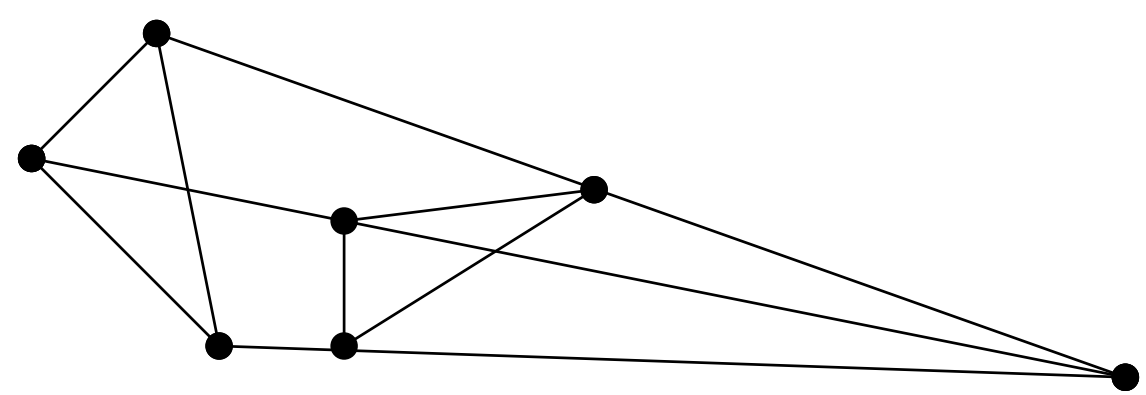

Figure 4

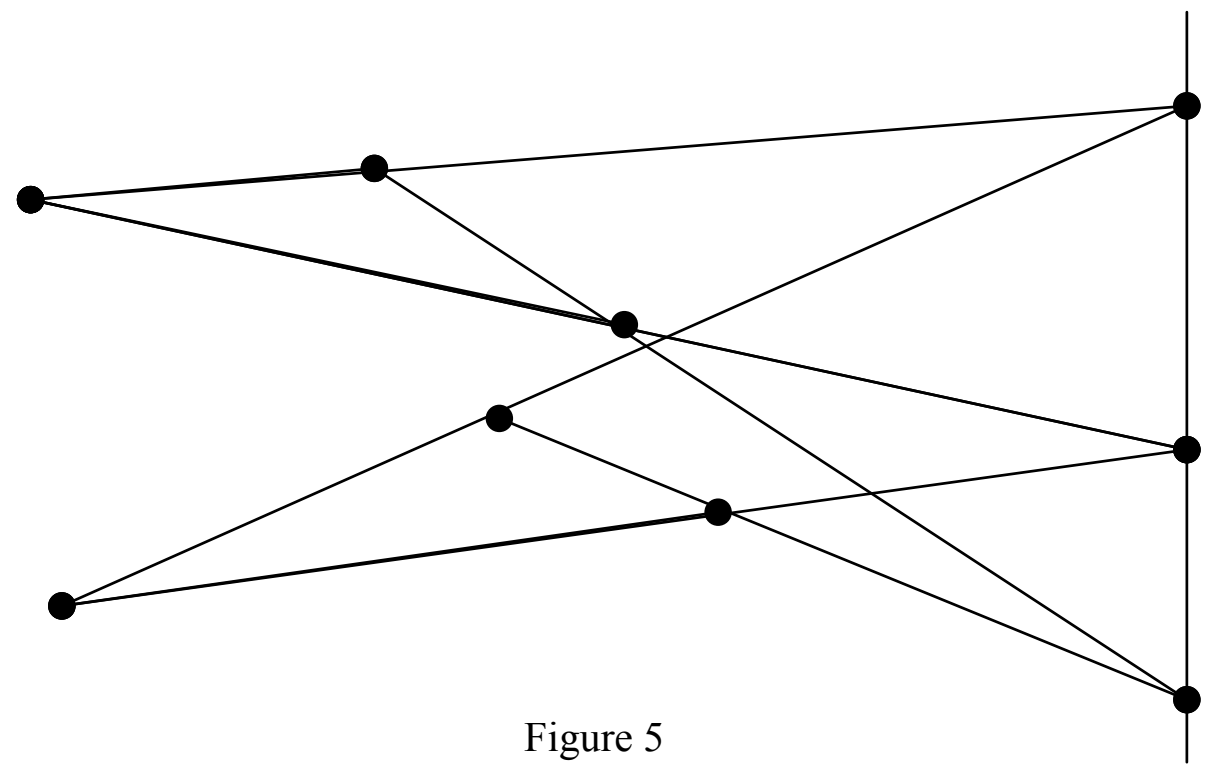

We may then state the theorem of Desargues: If two triangles are perspective from a point then they are perspective from a line, and conversely.

The notion of harmonics is central to the way we will be building our system of coordinates, and two definitions are needed to define this concept. Four collinear points $A, B, C, D$ form a harmonic set if a complete quadrangle can be constructed such that the intersections of two of pairs of opposite sides are at $A$ and $B$ respectively, and the two remaining sides pass through $C$ and $D$ respectively. We say that $C$ and $D$ are harmonic conjugates of each other with respect to $A$ and $B$ (figure 6). 


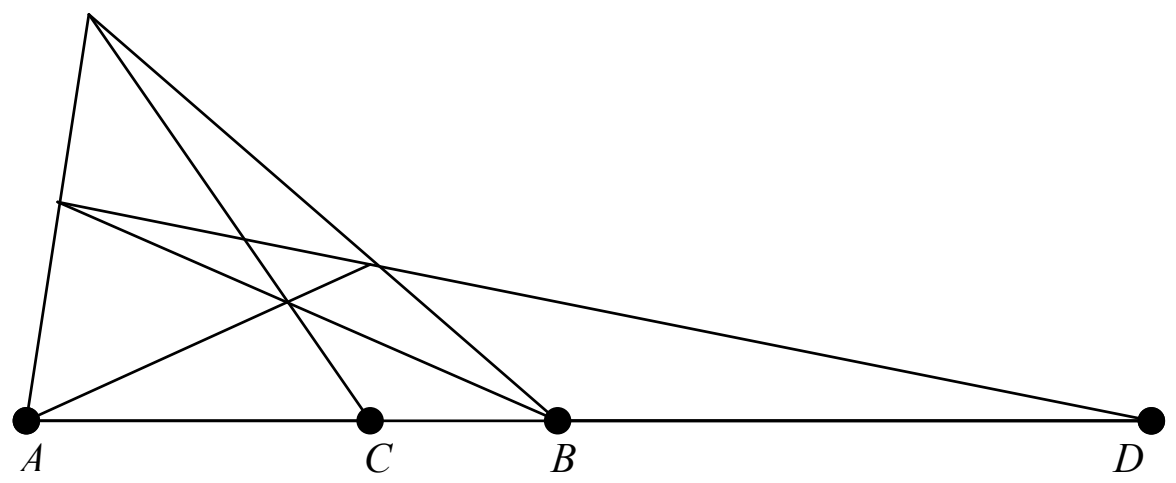

Figure 6

Before deriving a system of coordinates for projective geometry, we should consider in detail the notions of perspectivity and projectivity that are central to this study. As has been mentioned above, projective geometry has its historical origins in the work of the Renaissance artists and their desire to project a physical scene onto a canvas. Consider an artist drawing the patterns of a tiled floor onto a canvas. The method that was developed was to consider the lines going from the artist's eye, through the canvas, to points on the floor. Where this line intersects the canvas is where a mark should be drawn that corresponds to the point on the floor. I assume that the reader is familiar with this method, and a lengthy description is not required.

For the sake of simplicity I will restrict our mathematical analysis to plane geometry wherever possible. The extra work required to extend our system to cover three dimensional space is slightly more challenging mathematically as it increases the complexity of our system, however, there are no philosophical issues raised by extending the analysis. Therefore I will assume that if we can develop a coherent and comprehensive system of plane geometry based on the primitive actions outlined above, then it would be possible, with a little mathematical effort, to extend this system to three dimensions. Even within the plane we may perform a variety of interesting projections. Indeed, it is the study of these projections that is central to projective geometry.

Within our formal system, a projection is considered to be a kind of transformation that maps one collection of lines and points onto another. For example we may 
consider the lines that form the triangle $A B C$ below and the mapping of this triangle to the triangle $A^{\prime} B^{\prime} C^{\prime}$ from centre $O$ below.

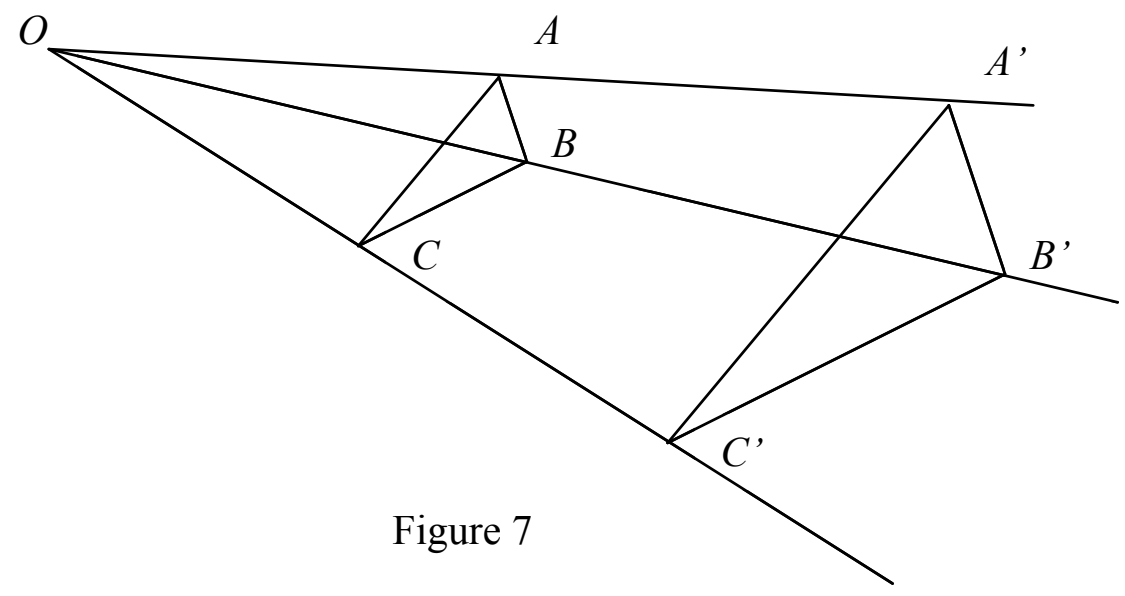

This particular transformation is an example of a perspectivity with centre $O$, it also happens to be a dilation scale factor two. The perspectivity need not transform $A B C$ onto a similar triangle, but the collection of all such perspectivities clearly includes all dilations of the triangle about the given centre. More formally, we may define a perspectivity with centre $O$ as a transformation from points $X$ on line $p$ and points $X$, on line $p$ ' such that the line $X X^{\prime}$ goes through the fixed point $O$. Dually, we may define a perspectivity with axis $o$ as a transformation from lines $x$ through point $P$ to the lines $x^{\prime}$ through point $P^{\prime}$ such that the intersection of $x$ and $x$ ' lies on the fixed line $o$. The transformation above is actually three perspectivities, one for each line.

A projectivity may now be defined as a transformation that is made up of a finite sequence of perspectivities. By stringing together finite sequences of perspectivities we may create a myriad of mappings including dilations, expansions, reflections, rotations, shears, stretches and translations. These transformations need not be defined as primitive operations as it should be clear that given the primitive operations we have in place, we, or at least our ideal agent, will be able to perform these actions. (I believe that it is significant that this method allows us to map an object onto its reflection without picking the object up, taking out of the plane, flipping it over, and putting it back down.) 
After the Renaissance artists perfected the process of performing projections, the question then arose, what properties of the physical object are preserved by projection. For the artist this meant, what properties will be the same no matter where the artist's eye is, (i.e., by taking different projections of the same object), or where the screen is placed, (i.e., by taking different sections of the same projection). "In other words, the mathematicians were stimulated to seek geometrical properties common to all sections of the same projections of a given scene. This problem is essentially the one that has been the chief concern of projective geometers in their development of their subject." (Kline 1956, p. 626) We are correspondingly concerned with the properties that remain invariant under projectivities.

It is clear that some properties must remain the same "since we could not otherwise recognize the projection as being a true picture of the original figure." (Hilbert \& Cohn-Vossen 1952, p. 94)

The things that vary after performing the projections as carried out by the artists, and hence cannot be considered to be projective properties include: the lengths of lines, the size of angles, the size of areas, and parallelism. The things that are preserved are: straightness, incidence, triangularity, quadrilateralarity, etc., and, interestingly, Pappus' theorem always holds, and "every projection or section of a harmonic form is itself a harmonic form." (Cremona 1913, p. 43) These are precisely the properties that remain invariant under the transformations as performed within our system outlined above and are labelled as projective properties.

We may now derive a system of coordinates for the projective plane. It is important to realise that these coordinates neither give immediate rise to, nor are derived from, a system of measurements. "This is apt to give rise to confusion, especially in the mind of the student, who fails to realise that the quantities used, so far as the propositions are really projective, are mere names for points, and not, as in metrical geometry, actual spatial magnitudes.” (Russell 1956, p. 28)

To develop the coordinate system we begin by drawing a straight line. We choose three arbitrary points on the line and label them 0,1 and $\infty$. We then find the harmonic conjugate to the first with respect to 1 and $\infty$ and label this point 2 . This 
method is purely constructive in nature and requires that we are able to construct a complete quadrangle given the intersection of two of its opposite sides and a point through which one of its remaining sides passes. This is entirely within the constraints of our system of geometry.

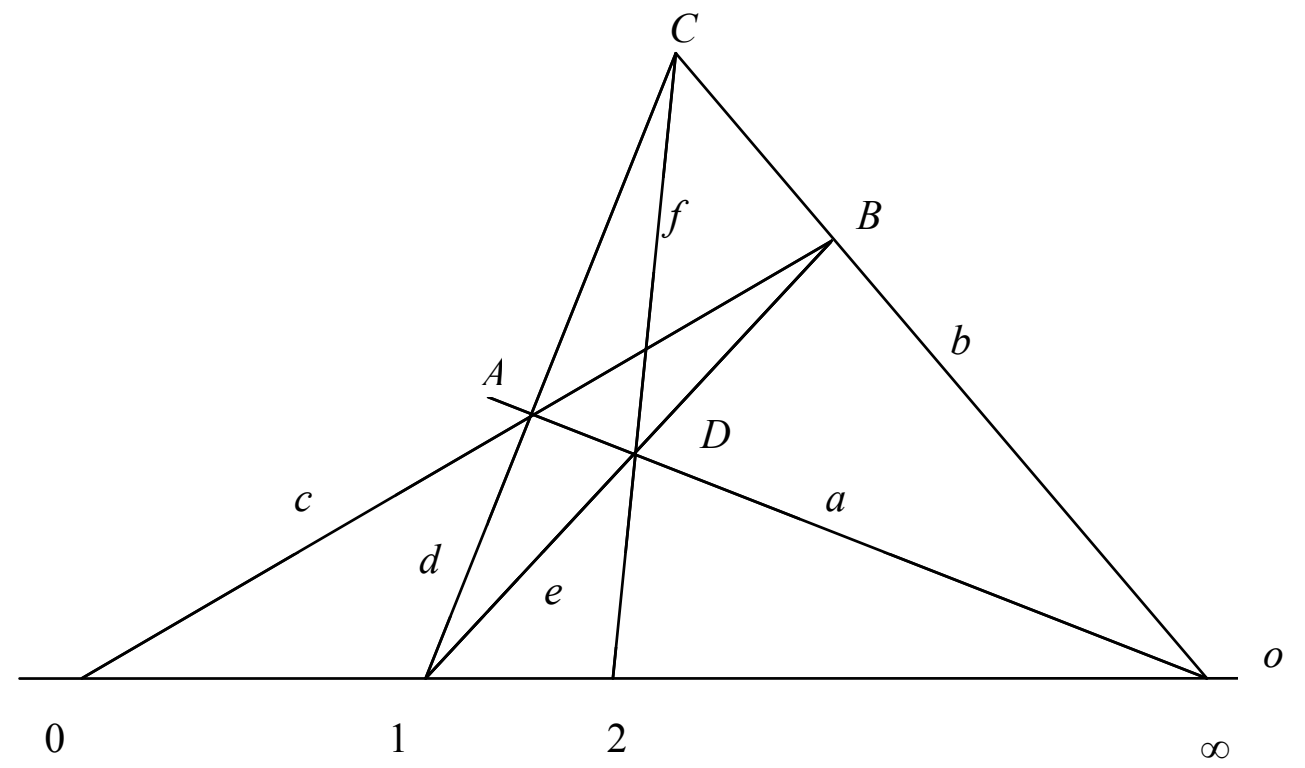

Figure 8

To check that this construction is in fact always possible within the system of geometry as we have developed it, consider the following procedure: Draw a line and label it $o$. We label three points on the line 0,1 and $\infty$ arbitrarily, remembering that our first theorem, above, guarantees that this line is incident with at least three other lines, hence has at least three points lying on it. Each point in our geometry is incident with at least three lines (by P3), therefore we may draw two more lines through point $\infty$, label these lines $a$ and $b$. Draw a line, $c$, through point 0 , this line is guaranteed to intersect both $a$ and $b$ (by P1), call these intersections $A$ and $B$ respectively. Join the points 1 and $A$ (by $\mathrm{P} 2$ ), labelling the line $d$, and join the points 1 and $B$, labelling the line $e$. Line $d$ must intersect $b$ and line $e$ must intersect $a$ (by P1), label these intersections $C$ and $D$ respectively. Then draw a line through points $C$ and $D$ (by $\mathrm{P} 2$ ) and label this final line $f$. Line $f$ must intersect line $o$, (by P1) and it is this point that we label 2. It should be clear that $A B C D$ form a complete quadrangle, and that $1, \infty, 0,2$ form a harmonic set, with 0 and 2 harmonic conjugates with respect to 1 and $\infty$. That the intersection of the last remaining side of the quadrangle and the original line is always the same regardless of how we construct our complete quadrangle is provable as a theorem within our system. (Tuller 1967, p. 41) It is 
important to note that this construction of coordinates along a line results from operations that an agent (ideal or otherwise) may carry out simply by drawing lines.

We then find the harmonic conjugate of the point 1 with respect to 2 and $\infty$, and label this point 3 . We, or at least our ideal mathematician, may continue this process indefinitely locating points on the line corresponding to any positive integer no matter how large. We may also generate negative and rational coordinates. In fact, "Klein has shown that by this construction, we can obtain any number of points, and construct a point corresponding to any given number, fractional or negative." (Russell 1956, p. 32-3) Tuller refers to this construction as a "net of rationality". (Tuller 1967, p. 52)

By introducing such a numerical system onto two distinct lines we may arrive at a coordinatisation of the plane, and we have done so by purely constructive and descriptive methods, with no presupposition of the measurement of distance whatever. The system we derive is that of homogeneous coordinates, assigning an ordered triple of numbers $\left(x_{1}, x_{2}, x_{3}\right)$, not all zero, to each point with the rule that $\left(\mu x_{1}, \mu x_{2}, \mu x_{3}\right)$ is the same for any $\mu \neq 0$, and a line is a homogeneous linear equation. It is worth noting that $x_{\alpha}$ and $\mu$ are not necessarily real numbers, if we have assumed that Pappus' theorem holds (which we have), any field will do.

What this coordinatisation does not give us, however, is a betweeness relation in the usual sense. Given three points on a line, say 0,1 and 2, we cannot say that 1 lies between 0 and 2. This kind of betweeness is not a part of projective geometry as it is not preserved under projection. (In fact any three collinear points can be projected onto any other three collinear points.) What we do have is a kind of separation relation. Given four points $0,1,2,3$ for example, we may say that one pair of points, 0,2 , separates the other pair, 1,3 , which we write $02 / / 13$. This means that one point from the first pair may be considered to be "between" the other two points, but which point it is will vary under projection. Tuller introduces "axioms of order" to define the relation of separation, and then proves that the pairs of a harmonic set, such as 0,1,2,3, separate each other. (Tuller 1967, p. 48-9) But these axioms are not required to develop the net of rationality described above. 
We may therefore not consider this ordering to be linear, but rather cyclic. In fact a one-to-one correspondence can be set up between points on a projective line and points on a Euclidean circle. This adds fuel to the notion of if you continually produce a line in one direction then you will arrive at your starting point from behind. However, projective geometry cannot endorse this view as it lacks a notion of sidedness, two points are always on the same "side" of any given line.

This also means that any kind of distance relation we develop based on this system will be a relation between four points. The distance between any two points will be derived with respect to another two points:

"The projective geometer, therefore, sees no reason to give a name to the relation between two points, in so far as this relation is anything over and above the unlimited straight line on which they lie; and when he introduces the notion of distance, he defines it, in the only way in which projective principles allow him to define it, as a relation between four point." (Russell 1956, p. 34)

If we want to determine the distance between two points on a given line in the projective sense, we first need to decide on our points 1 and $\infty$. Then we can use one of the two points as the 0 point, and use the above procedure to determine the distance from this point to the other. This will work, provided that the distance is not irrational, but will not satisfy Russell:

"Distance in the ordinary sense is, in short, that quantitative relation, between two points on a line, by which their difference from other points can be defined. The projective definition, however, being unable to distinguish a collection of less than four points from any other on the same straight line, makes distance depend on two other points besides those whose relation it defines. No name remains, therefore, for distance in the ordinary sense, and many projective geometers, having abolished the name, believe the thing to be abolished also, and are inclined to deny that two points have a unique relation at all. This confusion, in projective geometry, shows the importance of a name, and should make us chary of allowing new meanings to obscure one of the fundamental properties of space.” (Russell 1956, p. 36) 
Two issues need to be addressed here. Firstly, given that we have known since antiquity that certain lengths, such as the diagonal of a unit square, cannot be expressed by the rational numbers, can our system be taken one step further to cover the whole real line? And secondly, whether or not Russell's criticism that the projective notion of distance is not "distance in the usual sense" is fatal to our system. I will tackle these issues in that order.

We may define "betweeness" as a relation between three points and $\infty$, as follows, given three collinear points $A, B, C$, we say that $C$ is between $A$ and $B$ iff $A B / / C \infty$. With this definition of betweeness we may apply Dedekind's postulate of continuity to provide an isomorphism between the set of real numbers and the projective line less the point $\infty$. This should satisfy the first question.

The question remains open as to whether we have generated an adequate definition of distance. Russell feels that the arbitrariness of the selection of the unit point, 1, and the point at infinity, $\infty$, leads to conventionalism. However, I am not convinced that this is any different from arbitrarily choosing a rigid rod as a unit length, which seems to be the only other natural option.

If we are to use our system to determine the distance between two points, $A$ and $B$, on a given line, then we first need to place our unit point, 1 , and our point at infinity, $\infty$, on the line. We then treat the point $A$ as the zero point and follow the procedure and see how many times we need to repeat it to get to point $B$. This is effectively projecting the part of the line between $A$ and 1 onto the line so that the start of the next projection coincides with the end of previous projection, and continuing until we reach $B$. This is slightly over simplified, because it assumes an integer distance, but for rational and irrational distances the procedure is well defined and is essentially the same. The choice of where to put the unit point is arbitrary. But the decision of how long the unit length will be is an arbitrary decision in any system of measurement. The only salient issue is the placement of $\infty$.

The choice of where to put $\infty$ determines the kind of geometry we are considering. We may observe that the farther away we place the point $\infty$, the closer our coordinates 
get to Euclidean coordinates. If we place $\infty$ literally "at infinity" then the lines $a$ and $b$ as constructed above will be parallel (in the Euclidean sense) to our original line. If we then carry out the same procedure described above, determining harmonic conjugates, then we get the usual notion of distance for the Euclidean plane. The point of interest, however, is that we have defined a procedure for determining distances that does not rely on superposition or the movement of rigid rods. Our procedure is carried out by performing projections which is done simply by drawing lines, the operation we took to be primitive.

In summary, we began by taking our primitive operation to be that of drawing straight lines. We observed that some lines intersect to form points, giving rise to an incidence relation and a notion of concurrence and collinearity. Our crude physical paradigm was taken to be the familiar operation of drawing lines with a straight edge and pencil. We then idealised this operation by considering how an ideal agent who is not restrained by our accidental physical limitations may perform such operations. Next, we made stipulations about our ideal agent's ability to perform these operations, P1 P6. Thus we are defining projective geometry to be descriptive of the line drawing operations that our ideal agent may perform given these parameters. What we have discovered is that our ideal agent can construct a coordinatisation of the projective plane and can give a notion of distance between any two points given an arbitrary unit point and a point at infinity. During this process we have made no existential claims about the prior existence of points, lines or planes. Our only ontological claim is that the worlds in which our ideal agent performs these line drawing operations may be meaningfully considered to be fictional worlds. These fictional worlds may be defined as any world in which a line drawing agent's ability to perform line drawing operations is constrained by the axioms P1 P6. Thus projective geometry may be said to be descriptive of the operations that an ideal may perform within such fictional worlds. There may appear to be circularity here because we are saying that projective geometry is the study of the things that an ideal agent may do within fictional worlds, and that the fictional worlds are defined as worlds in which an ideal agent may do certain things. However, the way in which we have defined the fictional worlds in question is by stating that such worlds are ones in which an ideal agent may perform certain operations axiomatically, the interesting part of geometry is to then determine what other operations the ideal agent must be 
able to perform. This conception of geometry thus captures the implicational nature of geometry.

What we have discovered is that if we consider worlds in which our line drawing agent has their abilities constrained by P1 $\sim$ P6, then we may form a coordinatisation of such a world using homogeneous coordinates and develop a notion of measuring distances that is based on the line drawing process of making projections. 


\section{$\S 4.3$ Erlanger Programme}

Perhaps the most significant feature of projective geometry is the privileged place it holds in the hierarchy of geometries. Projective geometry may be said to be the "super-geometry" and the familiar Euclidean, hyperbolic and elliptic geometries may be considered to be "sub-geometries" of it. This position at the top of the geometric hierarchy led Cayley to exclaim that "Projective geometry is all geometry." (Nerlich 1976, p. 639)

The way to develop these geometries from projective geometry is to consider various transformations, and the properties which remain invariant under these transformations. This approach was presented by Felix Klein who, in 1872, redefined geometry as the study of the properties that remain invariant under different groups of transformations. (Tuller 1967, pp 65) This treatment of geometry is known as the Erlanger programme, named after the University of Erlangen where Klein held a professorship. This branch of geometry has been extensively studied and documented, most notably by Felix Klein and Arthur Cayley, but to my knowledge nobody has written about the ontological significance of this approach. And I am almost certain that nobody has claimed that this approach has significant parallels to the work of Kitcher.

One of the reasons for taking this road is that if you follow Euclid's foundations of geometry, you will very quickly realize that one of the un-stated assumptions is the ability to move geometric configurations around. In his proof of the fourth theorem, side-angle-side congruence, which is the first non-constructive theorem, Euclid requires us to superpose one triangle onto another. It appears that Euclid was reluctant to employ this technique in proofs, because he avoided it wherever possible. However, the idea of introducing a notion of continuous or rigid motion is intriguing and has implications for measuring distant objects and the problem of conventionalism. The most elegant approach to a theory of motions that I know of is the theory of transformations based on Klein's Erlanger programme. This approach involves projecting shapes onto each other as a well defined method of superposing them. 
Another reason for the attraction to Klein's Erlanger programme is that transformations are operations that have their foundation in our empirical practices of performing physical geometry. In order to fit into our theory, the transformation groups that we will consider all need to have a physical paradigm from which we can create an idealisation.

To begin Klein's Erlanger programme we first need to define a transformation as a one-to-one mapping from one set onto another. The transformations that we will be interested in are mappings from one collection of lines onto itself. These transformations are operations which we are taking to be the idealisation of the physical paradigm of projectivities discussed in the previous section. Taking an operation to be the foundational element of our theory of geometry gives us the Kitcherian foundation we are after. We also define a transformation's inverse, as the transformation that takes the image back to the original figure. The product of two transformations is the result of performing one transformation and then performing another transformation on the image of the first. The identity transformation is a transformation that maps something onto itself.

If a collection of transformations from one set onto itself contains the identity transformation and is closed under multiplication, then it forms a transformation group, and can be considered a group in the algebraic sense. Any property which is unchanged under all the transformations of a transformation group is said to be an invariant property of the group.

If a subset of a set of transformations which form a transformation group also forms a transformation group in its own right, then it is called a subgroup.

“We are now ready for Klein's famous definition of geometry:

A geometry is the study of those properties of a set $S$ which remain invariant when the elements of $S$ are subjected to the transformations of some transformation group.” (Tuller 1967, pg 70) 
One of the other significant aspects of Klein's Erlanger programme is its analytical nature. Not only does Klein consider the collection of transformations as algebraic groups, but the projective transformations he considers are represented analytically by linear homogeneous transformations of coordinates. To do this we need to build an algebraic model for our projective geometry.

"With the introduction of homogeneous coordinates, it became possible to reduce theorems of projective geometry to algebraic equations in much the same way that Cartesian coordinates allow this to be done for the theorems of metric geometry." (Hilbert \& Cohn-Vossen 1952, p. 94)

The analytical model for projective transformations is obtained by considering a line to be defined as a class of ordered triples, $\left[u_{1}, u_{2}, u_{3}\right]$, of homogeneous coordinates where $[0,0,0]$ is excluded and $\left[v_{1}, v_{2}, v_{3}\right]$ is in the same class as $\left[u_{1}, u_{2}, u_{3}\right]$ if and only if $v_{i}=k u_{i}$, for some $k \neq 0$. A point is defined the same way and is denoted $\left(x_{1}, x_{2}, x_{3}\right)$. A point $\left(x_{1}, x_{2}, x_{3}\right)$ and a line $\left[u_{1}, u_{2}, u_{3}\right]$ are said to be incident if and only if $u_{1} x_{1}+$ $u_{2} x_{2}+u_{3} x_{3}=0$. When $x_{i}$ and $u_{i}$ are taken to be any real numbers then we will have a model of the real projective plane, but any given field would suffice. This is precisely the same system of coordinates that we developed through our constructive procedure outlined in the previous section.

Three points are said to be linearly dependent if and only if there exists a line with which they are all incident, otherwise they are said to be linearly independent. In terms of the model what this means is that given any three points $\boldsymbol{x}=\left(x_{1}, x_{2}, x_{3}\right), \boldsymbol{y}=$ $\left(y_{1}, y_{2}, y_{3}\right)$ and $z=\left(z_{1}, z_{2}, z_{3}\right)$ if there exists a line $\left[u_{1}, u_{2}, u_{3}\right]$ such that $u_{1} x_{1}+u_{2} x_{2}+$ $u_{3} x_{3}=0, u_{1} y_{1}+u_{2} y_{2}+u_{3} y_{3}=0$ and $u_{1} z_{1}+u_{2} z_{2}+u_{3} z_{3}=0$ where $u_{1}, u_{2}$ and $u_{3}$ are not all zero, then the three points are collinear and are said to be linearly dependent. If, however, the only way to satisfy all three equations is to have $\left[u_{1}, u_{2}, u_{3}\right]$ equal to $[0$, $0,0]$, then the three points are not collinear and are termed linearly independent. It is a result of linear algebra that a necessary and sufficient condition for these three equations to have a solution such that not every $u_{i}$ is zero is for the rank of the matrix $[\boldsymbol{x}, \boldsymbol{y}, \boldsymbol{z}]$ to be less than 3 . This result can be extended to a collection of any number of points. 
A projectivity or collineation for the plane is determined by a 3 by 3 non-singular matrix, $\boldsymbol{T}$. Given any point $\boldsymbol{x}$, the projection of $\boldsymbol{x}$ is defined as $\boldsymbol{x} \boldsymbol{T}$ using the standard laws of matrix multiplication. Since $\boldsymbol{T}$ non-singular it has an inverse and multiplying the projection of $\boldsymbol{x}$ by the inverse of $\boldsymbol{T}$ will give the original point. It follows from the laws of linear algebra that linear dependence, or independence, is preserved by projectivities, and hence projectivities are also termed non-singular linear transformations. Also, because the product of two non-singular matrices is itself a non-singular matrix, the product of projectivities is also a projectivity.

It should be clear that projectivities as defined above form a group. Projective geometry may then be defined as the study of the properties that are invariant under projectivities.

"For our purposes, however, we shall consider only the point-to-point transformations (and the induced line-to-line transformations) given by the group of non-singular collineations in the plane. Using Klein's concept of geometry we shall define real projective geometry of the plane as the study of properties invariant under this group and shall call such properties projective properties. These include the incidence relations and hence collinearity of points and concurrence of lines; the cross-ratio of four elements and hence the separation and the harmonic relations; the property of a subset of points or lines being a conic and the pole-polar relationship.

However, we cannot distinguish one type of conic from another nor one type of quadrangle from another. ... In the next chapter we shall consider subgroups of the projective group which lead to geometries in which these distinctions can be made and which lead eventually to Euclidean geometry." (Tuller 1967, pp 102-3)

Basing our theory of geometry on transformations fits nicely with Kitcher's approach to mathematics. Remember that Kitcher avoided the "plight of the Platonist" by basing his theory of mathematics on the operations which mathematicians perform rather than on abstract entities. Here we may easily claim the same benefit found in Kitcher because transformations are clearly operations. We may demand a Kitcherian 
story as to how we learn to perform these operations of linear algebra, and I will outline briefly how this could be done.

By starting from the study of geometry in the ancient world, most notably in the work of Pappus, we may find an epistemological chain leading to the studies of the Renaissance artists and through a chain of mathematicians from Desargues to Poncelet to von Staudt to Klein. Although a student today may learn the techniques of linear algebra without knowledge of the physical paradigm outlined in the previous section, it was the theory of linear homogeneous transformations of coordinates which led to the development of algebra of matrices in the nineteenth century. (Todd 1947, p. 2) Therefore even though the student may or may not learn to perform these transformations through actions carried out in the physical paradigm based on drawing lines, if we follow Kitcher's thesis, there is an epistemologically significant link between the actions of drawing lines and the operations of linear algebra. This gives us the link to empirical reality that is required to explain the success linear algebra has with making predictions about the states of affairs in the physical world.

Our analytical model assumes that we have access to a set of symbols that satisfy the properties of a field. However, these symbols do not need to refer to any abstract entities or even to spatial points for the theory to work. What is important to our theory are the operations, the transformations, that we may perform. It seems perfectly reasonable to assume that the theory of arithmetic that Kitcher developed could easily be extended to linear algebra without the need to postulate the existence of abstract entities. Therefore, even though I have set out the analytical model in the usual way that appears to assume the existence of a collection of abstract entities, with a little work, and a lot of space, this could be done in a Kitcherian fashion, and the key role played by transformations in Klein's theory lends itself to this kind of development.

We now need to explain the connection between the synthetic world outlined in the previous section and the analytical model outlined above. We may easily show that this model satisfies the axioms for projective geometry P1 P6. This is carried out by Tuller (Tuller1967, pp 72-75) and will not be reproduced here. The important point that this demonstrates is that if we consider the analytical model to be 
representative of a possible world, then anything that holds true for lines and points in this possible world will also hold true for lines and points in the world of analytic projective geometry outlined above. We therefore have an analytical means of determining the truth or falsity of statements in our synthetic world. Because the nature of our synthetic world is linked to reality in an epistemologically significant way, knowledge obtained by the analytical method also carries an epistemological link to reality. This connection is vital in explaining the success of geometry.

Following Kitcher's suggestion as to how we can apply a possible world approach to mathematical theories we shall call any world which has a stock of operations which satisfy the axioms of projective geometry $(\mathrm{P} 1 \sim \mathrm{P} 6)$ P-worlds. Furthermore, I will call something which is true in every P-world a $P$-world truth. The world generated by the analytical model satisfies the axioms, as was shown by Tuller, and therefore is a P-world. We can therefore be sure that every P-world truth holds true in that model, but not that every truth of the model is a P-world truth as there may be hidden restrictions in the analytical model. We really need both a completeness and a consistency proof to show that all $\mathrm{P}$-world truths and only $\mathrm{P}$-world truths are true of the model before we can be truly happy to proceed. I will assume that this is possible, and that truth in the analytical model corresponds exactly to truth in the synthetic world.

The next, and indeed defining, step of the Erlanger programme is to determine certain subgroups of the group of projective transformations.

A collineation in the real projective plane that leaves a given line invariant is called an affine transformation. Any line of the real projective plane may be selected to serve as this invariant line and is called the ideal line or the line at infinity and is denoted $l_{\infty}$. Points on $l_{\infty}$ are called ideal points or points at infinity. All other lines and points are called ordinary lines and ordinary points, but we will assume that the words "line" and "point" refer to ordinary points unless otherwise specified. The set of all affine transformations forms a group and is called the affine group and is a subgroup of the projective group. According to Klein's definition of geometry, the study of properties which are invariant under affine transformations is called affine geometry. 
For convenience of notation we shall choose the line with the equation $x_{3}=0$ to be $l_{\infty}$. We then seek an analytical expression for the collineations that leave $x_{3}=0$ invariant. A little investigation will reveal that any transformation matrix of the form $\boldsymbol{T}=$ $\left(\begin{array}{lll}t_{11} & t_{21} & 0 \\ t_{12} & t_{22} & 0 \\ t_{13} & t_{23} & 1\end{array}\right)$ will give an affine transformation. Further investigation will show that affine transformations form a group.

Under affine transformations any triangle may be mapped to any other triangle so any two triangles may be considered to be equivalent in affine geometry. Two ordinary lines are defined to be parallel if and only if they intersect at an ideal point. It may be proven that parallel lines correspond to parallel lines under affine transformations, therefore parallelism is an affine invariant, and is said to be an affine property.

We may also distinguish between three classes of conics in affine geometry, parabolae, hyperbolae and ellipses according to their relation to the line at infinity.

Similarity transformations are the next kind of transformations to be considered. A similarity transformation is an affine transformation that not only leaves $l_{\infty}$ invariant but also leaves a certain pairing of points on $l_{\infty}$ invariant. Without going into too much detail this pairing of points is defined by a given elliptic involution on $l_{\infty}$, a mapping, other than the identity, whose square is the identity and that has two invariant points. A similarity transformation is then defined as an affine transformation that leaves the given elliptic involution invariant. These transformations take the form $\boldsymbol{T}=\left(\begin{array}{ccc}t_{11} & t_{12} & 0 \\ -t_{12} & t_{11} & 0 \\ t_{13} & t_{23} & 1\end{array}\right)$ or $\boldsymbol{T}=\left(\begin{array}{ccc}t_{11} & t_{12} & 0 \\ t_{12} & -t_{11} & 0 \\ t_{13} & t_{23} & 1\end{array}\right)$ and the set of similarity transformations forms a subgroup of the affine group.

As the name suggests we may begin to talk about angles. We may define perpendicularity as a similarity property. Furthermore, we may define the cosine of an angle, which is an invariant property under similarity transformations. We may also define acuteness of an angle. 
Homothetic transformations are the next transformations to be considered.

Homothetic transformations leave $l_{\infty}$ pointwise invariant and are given by a transformation matrix of the form $\boldsymbol{T}=\left(\begin{array}{ccc}t_{11} & 0 & 0 \\ 0 & t_{11} & 0 \\ t_{13} & t_{23} & 1\end{array}\right)$. The set of homothetic transformations form a group.

We may use homothetic transformations to define translations, dilations and, using harmonic homologies, orthogonal line reflections.

We may then define a distance function, or metric, for the plane, and consider the set of transformations that keep distance invariant, known as Isometries, or rigid motions. These include displacements, symmetries and rotations which are the similarity transformations that preserve length. If we define our metric in the usual Euclidean way, using the theorem of Pythagoras, then we will have succeeded in developing a theory of Euclidean geometry. We therefore define an isometry to be a transformation matrix of the form $\left(\begin{array}{ccc}t_{11} & t_{12} & 0 \\ -t_{12} & t_{11} & 0 \\ t_{13} & t_{23} & 1\end{array}\right)$ or $\left(\begin{array}{ccc}t_{11} & t_{12} & 0 \\ t_{12} & -t_{11} & 0 \\ t_{13} & t_{23} & 1\end{array}\right)$ where $t_{11}{ }^{2}+t_{12}{ }^{2}=1$. However, our choice of metric was entirely arbitrary and more needs to be said about the way the metric can be introduced. Indeed, this brings us to the heart of the Erlanger programme.

Following the work of Cayley and Klein we may introduce the metric into projective geometry in terms of a conic, called the absolute, to be kept invariant. We will find that the resulting geometry will either be hyperbolic, Euclidean or elliptic depending on how we determine this conic. By taking the absolute to be an imaginary conic we may obtain elliptic geometry. By taking the absolute as a real conic we obtain hyperbolic geometry, and by taking the absolute as a degenerate conic in the form of a line counted twice we may obtain Euclidean geometry. 
We will need to incorporate complex numbers as an aid to our development: A complex point is an ordered triple of complex numbers $\left(x_{1}, x_{2}, x_{3}\right)$, not all zero, where the point $\left(x_{1}, x_{2}, x_{3}\right)=\left(k x_{1}, k x_{2}, k x_{3}\right)$ for any complex number $k \neq 0$. This does not mean that we will be considering the imaginary plane; we will still be considering only the real projective plane as our super-geometry.

By choosing our coordinate system in the appropriate way we express a real nondegenerate conic by the equation $x_{1}^{2}+x_{2}^{2}-x_{3}^{2}=0$, an imaginary conic by the equation $x_{1}^{2}+x_{2}{ }^{2}+x_{3}^{2}=0$, and a degenerate conic by the equation $x_{3}^{2}=0$. All three equations may be expressed in the form $c\left(x_{1}^{2}+x_{2}^{2}\right)+x_{3}^{2}=0$, where $c=-1,+1$ or 0 to give a real, imaginary or degenerate conic respectively.

Taking the absolute to be a real non-degenerate conic we define the points of the hyperbolic plane to be the set of points of the real projective plane which are interior to the absolute conic, and lines to be chords of the conic. Two lines are defined to be parallel if and only if they intersect on the absolute conic. Two lines are called ultraparallel if and only if they intersect outside the absolute conic. Two lines are perpendicular if and only if they are parts of projective lines which are conjugate with respect to the absolute conic i.e., if each passes through the pole of the other.

Within the hyperbolic plane defined above we may prove, among other things, that any two points determine a line, any two lines determine at most one point, there are exactly two parallels to a given line through a point not on that line, and there is exactly one line perpendicular to a given line through a point on that line.

Distance in the hyperbolic plane is given by the natural logarithm of a cross-ratio. If we take two points, $A, B$, of the hyperbolic plane we may determine a unique line incident with both points. This unique line intersects the absolute conic at exactly two places, $P, Q$. We define the distance from $A$ to $B$ to be the absolute value of the natural logarithm of the cross-ratio of $A, B, P, Q$ multiplied by an arbitrary constant. If we fix the order of $P$ and $Q$ in the cross ratio and omit the absolute value then this gives a directed distance for $A B$. The arbitrary constant is chosen according to the unit of length we desire. 
Any transformation that keeps the absolute conic invariant is considered to be a hyperbolic transformation and the properties that remain invariant under hyperbolic transformations are considered to be hyperbolic properties. A particularly interesting hyperbolic transformation is a harmonic homology (a collineation with one invariant point $P$ and one line $p$ of invariant points, where $P$ does not lie on $p . P$ is called the axis of the homology and $p$ is called its centre) whose centre and axis are pole and polar with respect to the absolute conic. Such a transformation forms an isometry, or rigid motion in the hyperbolic plane.

Taking the absolute to be an imaginary conic we define the points of the elliptic plane to be the points of the real projective plane that are not on the conic, i.e., all the points of the real projective plane, and similarly, the lines of the elliptic plane are all the lines of the real projective plane.

Within the elliptic plane we may prove, among other things, that two points determine a unique line, that two lines determine a unique point, and that all lines which are perpendicular to a given line intersect at a point. To determine a metric for the elliptic plane we consider any two points $A, B$ and the unique line determined by them. The intersections, $P$ and $Q$, of this line with the absolute conic are conjugate imaginary points, therefore the arbitrary constant in the distance formula must be a pure imaginary number to give a real value for distance. The distance from $A$ to $B$ is given by the natural logarithm of the cross-ratio of $A, B, P, Q$ multiplied by $-k i / 2$, where $k$ is a positive real number that it chosen according to the unit of length. An interesting result is that the length of any line is finite and is equal to $k \pi$.

To develop the Euclidean plane we take the absolute conic to be the line $x_{3}=0$ taken twice, which is a degenerate conic and may be called $l_{\infty}$, and consider two points, $I$ and $J$, which lie on this line and call them absolute points. We define points in the Euclidean plane to be points on the projective plane that do not lie on the absolute conic, i.e., the points of the affine plane. We define lines of the Euclidean plane to be lines of the real projective plane that do not pass through $I$ and $J$, i.e., all lines of the project plane except $l_{\infty}$. 
If we take the points on $l_{\infty}$ that remain invariant under similarity transformations to be $(i, 1,0)$ and $(-i, 1,0)$ and the distance between two points $\left(x_{1}, x_{2}, x_{3}\right)$ and $\left(y_{1}, y_{2}, y_{3}\right)$ is given by the formula $\frac{\sqrt{\left(y_{1} x_{3}-y_{3} x_{1}\right)^{2}+\left(y_{2} x_{3}-y_{3} x_{2}\right)^{2}}}{x_{3} y_{3}}$ then we have a geometry that conforms to all the Euclidean norms. The distance formula does not, however, conform to the standard requirements of a metric when it is used for imaginary points.

Therefore we should now be able to understand Cayley's statement that "projective geometry is all geometry" because we have been able to develop Euclidean, hyperbolic and elliptic geometry from the foundation of projective geometry. This also makes it clear why Russell put forward the notion that projective geometry provides the a priori foundation of all geometry. However, in contrast to Russell, we have based our knowledge of projective geometry on our empirical investigations, and the empirical investigations of our predecessors, and therefore find that projective geometry forms the quasi-empirical foundation of all geometry.

It should be clear that having formed projective geometry as an idealisation of our operations involving drawing straight lines, and having formed Euclidean, hyperbolic and elliptic geometries out of projective geometry, that all of these geometries may be understood as an idealisation of our operations as well.

We have already developed projective geometry as the idealisation of the operations that we may perform with a straight edge and pencil. In order to answer the ontological question we took the worlds in which our ideal agent operate to be fictional worlds similar in kind to the fictional worlds generated by literary fictions, or more closely to the fictional worlds generated by role-playing games. We therefore concluded that projective geometry is descriptive of the operations which an ideal agent may perform in a certain class of fictional worlds. We now find that using projective geometry as our base we may constrain our ideal agent's ability to perform operations in various ways, generating different classes of fictional worlds for each set of constraints. We may constrain our ideal agent's line drawing operations in one way and arrive at a class of fictional worlds that correspond precisely to Euclidean geometry. We may, however, constrain the ideal agent's ability to perform line 
drawing operations in another way and arrive at the fictional worlds of hyperbolic geometry. Or we may offer another set of constraints that result in the development of elliptic geometry. We may therefore conclude that the study of geometry, as is conceived by the quasi-empirical-fictionalist approach, may be defined as the study of the operations that an ideal agent may perform in various classes of fictional worlds.

The fictional worlds themselves are defined by the operations that an ideal agent may perform within them. This is not circular. We state axiomatically that an ideal agent may perform a certain set of operations and define the fictional worlds to be worlds in which these operations may be performed. The work of the mathematician is then to determine what other operations the ideal agent may perform in all such worlds, i.e., to determine the theorems that apply to that class of worlds.

The only complicating factor is the purely analytic nature of Klein's approach to geometry. The operations that Klein has based the study of geometry on are linear transformations. When carried out in the analytical model, transformations are carried out by performing matrix multiplication. When carried out in the synthetic model, transformations are projections of lines to lines and points to points carried out with a straight edge and pencil. The analytical approach is an indispensable tool for the study of geometry, and it is important to note several things about its use. Firstly, that the study of matrix algebra was developed out of the study of homogeneous coordinates in the real projective plane, and these coordinates, as we have seen, have an empirical foundation based on our operations of drawing lines. Therefore, according to Kitcher's evolutionary approach to the epistemology of mathematics, we may say that matrix algebra has a quasi-empirical foundation. Secondly, according to our fictional world approach to mathematics, the world generated by the analytical model has a close relationship to the synthetic world of projective geometry developed in Section 4.2, in fact, the analytical model is said to represent the projective plane. Due to the relationship that exists between the two worlds, we may say that statements of analytical geometry correspond to statements of synthetic geometry, and although we may consider the true nature of geometry to be synthetic, we may use analytic means to determine truths about the synthetic world. 


\section{§.4 Conventionalism}

One popular view of the nature of space is conventionalism, in fact "many philosophers on the contemporary scene are conventionalists and certainly the great majority of those who write on the philosophy of space and time are." (Nerlich 1976, p. 99). This view was first stated by Poincare and later developed by Reichenbach in his 1958 book The Philosophy of Space and Time (Reichenbach 1957). The theory was developed in response to the acceptance of the non-Euclidean geometries of Lobatschewsky, Bolyai and Riemann, and the adoption of non-Euclidean geometry as a model for physical space by the scientific community in the form of general relativity. Previous philosophies of geometry had considered Euclidean geometry to have a special place, but by the 1950 s relativity theory had been widely accepted and Euclidean geometry no longer held a privileged position. The scientific theory of space had changed, and the philosophical view needed to be upgraded to match.

An important conclusion drawn by both Hempel and Reichenbach is that the plurality of geometries forces us to consider mathematical space and physical space as two separate entities. The logicists and the formalists believe that purely mathematical space has no subject matter. I have argued that it is preferable to say that this is not the case, rather, that mathematics is concerned with the actions that may be taken by an ideal agent operating within a certain class of fictional worlds. Therefore it is not the case that mathematics has no subject matter, but rather that the subject matter is not real, it is fictional. But I agree with the distinction between physical geometry and mathematical geometry. When we are doing physical geometry we are talking about the states of affairs in the real world. When we are doing mathematical geometry we are talking about the states of affairs in the fictional world generated by the system of geometry under discussion.

The relationship between mathematical geometry and physical geometry, as we have seen, is rather complicated. Hempel claimed that when applying mathematical geometry to physical geometry we assign meaning to the geometric terms and concepts by assigning a specific designatum to each of the primitive terms. Our mathematical statements then become physical statements which we may test 
empirically. Therefore, we should be able to turn each geometry into a physical geometry and test each empirically to find out which one best describes physical space. As Reichenbach put it, "mathematics reveals the possible spaces; physics decides which among them corresponds to physical space.” (Reichenbach 1958, p. 6)

However, the situation is not so simple. Recall the experiments undertaken by Gauss to see if the Euclidean theorem about the angle sum of a triangle held over large distances. Hempel asks us to suppose that the results of Gauss's experiment showed that the angle sum of the triangle was significantly different from two right angles, and asks what we would conclude? We would not necessarily reject Euclidean geometry as the discrepancy might have been caused by some force that deformed the light rays.

"It was [Poincare's] opinion that no empirical test, whatever its outcome, can conclusively invalidate the Euclidean conception of physical space; in other words, the validity of Euclidean geometry in physical space can always be preserved - if necessary, by suitable changes in the theories of physics, such as the introduction of new hypothesis concerning deforming or deflecting forces. Thus, the question as to whether physical space has a Euclidean or a non-Euclidean structure would become a matter of convention." (Hempel 1945, p. 15)

Poincare recognised that the test of physical geometry was not simply a choice between geometries, but between geometries combined with a set of presuppositions about the measurement of physical space. He believed, however, that the decision to preserve Euclidean geometry would always be made because of Euclidean geometry's greater simplicity.

"The test of a physical geometry $G$ always presupposes a certain body $P$ of non-geometrical physical hypothesis (including the physical theory of the instruments of measurement and observation used in the test), and that the socalled test of $G$ actually bears on the combined theoretical system G.P rather than $G$ alone." (Hempel 1945, p. 15) 
Poincare's belief that a physical test of a geometry is not a test of that geometry in isolation, but a test of the geometry combined with a set of physical assumptions, may have been correct, but his belief that Euclidean geometry would always be upheld despite experimental evidence was incorrect. Recall the 1919 experiments of Eddington and Cottingham where the light from a distant star appeared to have bent as it passed near the sun. The apparent bending of light rays can, as has already been stated, be explained in two ways. One way is to state that space is still Euclidean, but the force of gravity acted on the light ray causing it to bend. The other explanation is that space itself is bent, and the light ray travelled along a straight path through the curved space. These two explanations exemplify what Poincare meant by saying that we could always retain Euclidean geometry by postulating a deforming force. Physicists have chosen to adopt a combined theoretical system G.P with a nonEuclidean $G$ as the preferred theory.

"And indeed, just this situation has arisen in physics in connection with the development of the general theory of relativity: If the primitive terms of geometry are given physical interpretations along the lines indicated before, then certain findings in astronomy represent good evidence in favour of a total physical theory with a non-Euclidean geometry as part $G$. According to this theory, the physical universe at large is a three-dimensional curved space of a very complex geometrical structure; it is finite in volume and yet unbounded in all directions. However, in comparatively small areas, such as those involved in Gauss' experiment, Euclidean geometry can serve as a good approximative account of the geometrical structure." (Hempel 1945, p. 16)

Therefore we find ourselves in the situation of having two competing theories which offer the same empirical output. Reichenbach explains the problem by way of a mind experiment. He asks us to imagine two two-dimensional surfaces and creatures inhabiting these surfaces. The top surface is flat with a hemisphere in the middle and the bottom surface is everywhere flat. The inhabitants of the top surface are able to discover the shape of their surface by the use of measuring rods just as people living on the surface of the Earth can discover its curvature. The flat surface, however, has a force acting on it which makes their measuring rods deform in such a way that the geometric readings are identical to those on the surface above. Our first, natural, 
inclination is to say that the top surface is a "surface with a hump" and the bottom surface is "a plane which appears to have a hump." (Reichenbach 1958, p. 13) But, from the perspective of the inhabitants of the surfaces, is there any real difference?

If the force that deforms the measuring rods on the bottom surface deforms everything in the same way, then, according to Reichenbach, the force will not be detectable. If the inhabitants of the surface did not already know what their geometry was, then they would draw the same conclusions about the shape of their space as the inhabitants of the top surface. Would their conclusion be wrong?

Here Reichenbach introduces the notion of a universal force and a differential force. A universal force is one that acts on every object in the same way, and to which there are no insulating walls. All other forces are differential forces. "Then it can be said that only differential forces, but not universal forces, are directly demonstrable." (Reichenbach 1958, p. 13)

The point Reichenbach is making is this, if space is in fact flat and there is a universal force, such as gravity, which deforms all objects in the same way so as to make space appear curved, then, is there any way of knowing whether we live in a space that is curved, or in a flat space that appears curved? Furthermore, if there is no epistemological way of knowing the difference, then is the difference real and or meaningful?

The heart of the matter, according to Reichenbach, is the notion of two distances being "really equal." And he asks, "Is really equal a meaningful concept?" (Reichenbach 1958, p. 14) Reichenbach believes that our method of measuring distances is based on rigid rods such as the standard metre in Paris. He claims that the core question is "how do we know that two rods that are congruent when placed next to each other remain congruent when they are separated?" This isn't as silly, or counter-intuitive, as it first sounds. Imagine that I am holding two tubes of mercury that have a constant diameter but can vary in length. When I hold them together at the beginning of the experiment we observe that they have the same length. I put one on one side of the room next to the fire, and the other on the other side of the room next to a bucket of ice. We would expect that when they are in these positions that they 
will be of differing lengths, but if we bring them back together, provided we do this slowly enough, we expect to find that they are of the same length. So the two rods are congruent when touching, but incongruent when apart. How do we know that the same is not true for our wooden metre rulers?

What makes the mercury rods expand in the above thought experiment is heat. Heat is a force, so we can say that forces are capable of changing an objects length. But heat is a differential force, it affects different materials differently, and this makes it detectable. In fact, this is why a thermometer works: the heat affects the mercury differently than it affects the glass. But imagine a universal force, one that affects all objects of all materials the same. (Gravity is such a force, a wooden ball and a steel ball fall at the same rate). Would we be able to notice it?

Reichenbach's claim is not that there is no way of knowing whether two rods that are separated by a distance are congruent, but that this is not a question of fact. We define them to be congruent by convention.

The method used by Reichenbach is to describe the world in one way, using a geometry and a field of force, and then to redescribe the world in a compatible way using a different geometry and a different field of force. It would appear that any difference in geometry can be accounted for by proposing a different force.

One way to look at the situation is that we have different theories, each of which describes a different possible world. The best theory would then be the one that is closest to the actual world. These are competing theories of the one actual world. If one is correct then the other must be wrong. This is not Reichenbach's conclusion. Reichenbach claims that the two theories do not differ in factual content, they are factually the same, and they only differ in their definitions, so which one is correct is just a matter of convention.

The issue may be summarised as follows: There are (at least) two competing ways to describe the geometry of physical space, one where physical geometry has a curved structure, which I denote $G_{2}$, and another where geometry has a flat, Euclidean, structure and there is universal force acting on it that deforms rigid rods and pulls 
light from geodesic paths, which I denote $G_{1}+F$. But the conventionalist's conclusion is not that these are two alternative, competing, theories to explain the one true reality, nor that one or other, but not both, of the theories could be true. Nor is the conventionalist's conclusion that both of these theories describe different possible worlds. "Reichenbach proposes that both descriptions can be equally true of the very same set of facts. He does not mean just that they are competing theories which explain the same evidence - the same known facts. So far as facts go, known or unknown, the descriptions are claimed not to differ." (Nerlich 1976, p. 100)

I will now look at Nerlich's objection to Reichenbach's conventionalism. He begins by reformulating the conventionalist's stance in a way that is uncritical of the doctrine of conventionalism and in a way that most conventionalists, particularly Reichenbach, would agree with. He then points out some disagreeable features of conventionalism that his reformulation of the thesis highlights. Nerlich's approach is largely epistemological, and he states the following to explain what he takes the study of epistemology to be about:

"The idea that convention, not fact, makes up a great deal of what we say about the world began as part of an epistemological theory. Epistemology sometimes means the study of what men really do know and how they come to know it. We might find that we can show, in this subject, that it is not possible ever to be sure whether our space is Euclidean or not, whether our world contains any material bodies, or whether we are forever dreaming. Reichenbach did not see epistemology in this light. Nor do I. He is interested in space, not in our knowledge of it, or rather, he is interested in what spatial concepts can be made intelligible by us and what structure they have. He is not concerned with how sure we are which of these concepts apply to actual space. Conclusions about what we might (or could never) know are interesting in themselves, perhaps, but not relevant to the problem before us now." (Nerlich 1976, p. 101)

Nerlich's approach begins with the idea of a problem language and a standard language. The standard language is a regimented bit of English that everyone agrees on. It is a set of sentences the truth of which we are certain of and a set which is 
closed under logical operations. The standard language is taken to be completely factual and objective. We are not concerned with who knows it, or how they know it, but state that it is knowable in principle. The problem language is an extension of the standard language that also includes some statements the truth of which we are not so sure about.

\begin{abstract}
"Suppose we start epistemology by choosing a class of statements whose truth conditions we think we grasp, clearly and directly, in our simplest interactions with the world. ... We can make later problems with complex forms of sentences a lot simpler by beginning with a set of open sentences free of truth functions or quantifiers. It is assumed that these open sentences are homogeneous in topic.... The sentences are supposed to be true of things (satisfied by them) in a way ideally transparent to us. Then we take the smallest class of sentences generated from these which is closed under logical operations (truth functional and quantificational). Call this class of closed sentences the standard language. Then it is the basis from which the remaining concepts of the language are built.” (Nerlich 1976, pp. 101-2)
\end{abstract}

So our task is to explain the problem language in terms of the standard language. Using the standard language as a base, extra principles can be added as matters of convention to build up to a problem language description of the world.

\footnotetext{
"This means that a given standard language description, which is factual and objective, can be embedded in several very different problem language descriptions. The broader, problematic descriptions will characteristically be incompatible with one another, since they are fixed by differing conventional descriptions. This is Reichenbach's position.” (Nerlich 1976, p. 104)
}

Now, if we consider that at least some of what we want to have in our standard language are statements about medium sized physical objects, and if we recognise, as Nerlich claims we must, that medium sized physical objects are, at least partly, spatial in nature, then we must have some spatial component to our standard language. The question then arises: what should this spatial component of our standard language be? 
Nerlich draws our attention to the hierarchy of geometries, detailed above, and briefly outlines the underlying features of Klein's Erlanger programme.

"Various metrical geometries were found to be subgeometries of projective geometry. The situation was like this: projective geometry is the study of a certain set of projective properties $P_{I}$ which are invariant (hence subscript ' $I$ ') properties of items in a space subjected to a certain projective group of transformations $P_{T}$ (hence subscript ' $T$ '). Euclidean geometry studies a group of metrical invariants $E_{I}$ of the transformation group $E_{T}$, Hyperbolic geometry studies the invariants $H_{I}$ of the hyperbolic group $H_{T}$. The groups $E_{T}$ and $H_{T}$ are proper subgroups of $P_{T}$. Every transformation of $E_{T}$ or of $H_{T}$ is a transformation of $P_{T}$, but not vice versa. Conversely, invariants of the metrical geometries are a larger class than the projective invariants, which they obviously include. This sounds very like what we want. The standard language can be a meagre spatial vocabulary and the problem language a much richer one." (Nerlich 1976, p. 105)

In keeping with the previous sections, this interpretation of geometry makes the objects of our languages transformations. This means that we are not talking about physical spatial things, rather, we are talking about transformations, or mappings, and properties which are invariant under these mappings. These concepts can be defined in a Kitcherian way as they are based on operations that we may perform. Therefore we find that the Kitcherian quasi-empirical approach to geometry that I have put forward is compatible with Nerlich's standard language - problem language interpretation of conventionalism.

Even though the Erlanger programme offers a very elegant framework in which to present a conventionalist interpretation of geometry, Reichenbach does not use projective geometry as his standard language base. Instead, Reichenbach treats local topology as the most fundamental of all geometries. Even though topology is considered to be more general than projective geometry the hierarchy is not so neat. Projective geometry is not a proper sub-group of topology, the two overlap. But if we brush these technical difficulties aside for the moment, there is a lot about topology to recommend itself as the spatial component of our standard language. What this 
means is that we will have to add some conventions to topology in order to incorporate projective geometry into it before we add the metrical conventions that define Euclidean, elliptic and hyperbolic geometry detailed above.

\begin{abstract}
"We might say that the world is factually definite only up to a topological description of things. This would tell us which subspaces are contractible, what is enclosed by what, whether things touch, interact or overlap. But, in fact, there would be no objective question whether two objects are of the same size unless this happened to be topologically definite: for example, when both are touching along their lengths so that neither overlaps the other. The remaining metrical description of the world is wholly conventional, dictated by what we find elegant and simple to use. It is not and cannot be a matter of getting the facts right. There are no metrical facts, only metrical conventions." (Nerlich 1976, pp. 106-7)
\end{abstract}

One example of the idea that we can determine topological facts can be seen in Reichenbach's discussion of the rigid rod. Reichenbach holds that we may determine whether two rods are of the same length only when we hold them so that they are touching each other and observe that their ends do not overlap. This is a topological conception of congruence. But when the rods are apart we can longer perform such a test, and hence topology is unable to tell us if they remain same length or not.

"The conventionalist program for metrical geometry seems beautifully clear and definite then. We take our theory of the world to be factual in its topology, but conventional in its choice of lines, parallels and metric." (Nerlich 1976, p. 108)

However, everything is not quite so clear. Reichenbach does not use general topology as his foundation, he uses local topology. Given that we accept the conventionalist's model of standard language and problem language with regard to geometry, an argument is needed to show that we should draw the line at topology and not at, say, projective geometry. In fact, it would appear that Reichenbach wants to draw the line within the field of topology, between local topology and general topology. We need convincing that this is the best place. 
"But what about Reichenbach's conventionalist program for showing that the global topology of a space is not factual but conventional? ... Yet it is obvious enough what the answer has to be. We must preserve the topology of individual objects, but the global topology of the space need not be included in the standard language. So Reichenbach claims that we can regard the global topologies of the spaces described in the preceding chapter as mere conventions. They do not enter into the factual description of the topologies of the various objects that are in the space." (Nerlich 1976, p. 108)

Thus we find that one problem with conventionalism is the decision where the line should be drawn between the standard and the problem language. Reichenbach has chosen local topology, probably the weakest of all branches of geometry, and stated that this is all we can be factually sure about, anything stronger will involve the adoption of some convention or other. I believe that conventionalism would be far more elegant if we were to adopt projective geometry as its foundation, but, as Nerlich warns us, "no doubt it is always agreeable to operate graceful and powerful machinery, but we are concerned with truth, not pleasant exercise." (Nerlich 1976, p. 131) Therefore I will consider this issue to be little more than an aesthetic blemish on conventionalism. We could easily build a Kitcherian quasi-empirical model for topology using the action of bringing rods together to see if one overlaps the other as our crude physical paradigm, and then idealise this operation. In fact, Reichenbach often refers to the physical paradigm of manipulating rigid rods as the foundation of our geometrical knowledge. Also, we can still use the Erlanger programme to show us exactly what the conventional assumptions we are making are when we proceed to one geometrical outlook or another. Anyway, "it seems admirably satisfactory to select topology as the spatial part of a standard language.” (Nerlich 1976, p. 108)

Nerlich puts the salient issue of conventionalism into a nutshell by considering the problem in terms of possible worlds, which fits nicely with the fictional world view of mathematical theories described above.

"This is all a bit vague. It would be a long job to make the idea really precise but we can harden the edges of it a little by putting the matter again in the 
terms of possible worlds. The meaning of a general term (an open sentence) is its extension in all possible worlds. We can get a pretty firm grip on the structural ideas at issue here by giving the set theoretical account of a range of possible worlds and of how the entities in a world fall into the sets which are the extensions of general terms. Ordinarily, we suppose that we were dreaming up a new possible world whenever we imagine that we might include something new in the extension of a general term. Thus, imagining that Winston Churchhill was among those killed in the 1940 bombing raids on London is certainly imagining a different possible world. But if a person puts forward a conventionalist thesis about some bit of language, he is denying that this sort of change is at issue. To change the reference of problematic terms is not to envisage a different possible world. So when Reichenbach and Grunbaum envisage a different group of congruence classes of spatial intervals they are not imagining a different possible world, but only redescribing this one. When we envisage changes in the extension of standard language general terms, then we are imagining different possible worlds, which factually differ from one another. When we envisage changes in the extension of problem language general terms, then we are only noting the possibility of different conventions of redescription of this possible world." (Nerlich 1976, p. 111)

My first serious objection to conventionalism, which is not found in Nerlich, is that the conventionalist's standpoint is essentially that two (or more) different descriptions of reality can be factually the same. This seems tantamount to saying that the descriptions of the paths of the planets in the solar system using general relativity, using Newtonian mechanics, using epicycles, or using a theory about a breed of giant fairies that are making a mobile for their child, are all factually the same, providing, of course, that they all have the same observable outcome and all agree on local topology. It seems intuitively clear that descriptions involving giant fairies and those using a force called gravity are factually different.

Nerlich's first general objection to conventionalism is that it is too stifling and restrictive. He believes that it limits what is knowable about the world too much. Conventionalism claims that various widely accepted theories misdescribe the world by over-determining it. "What is interesting in what [Reichenbach] says is that the 
theory as we ordinarily take it, at face value, misleads us quite strikingly as to the objective structure of things." (Nerlich 1976, p. 128)

"Further, if the complexity of the world can outrun the complexity of the standard language, then extensions of language beyond the scope of the standard language can be regarded as speculative hypotheses about which facts lie beyond the pale of the preferred primitive ones. But then it would be in principle utterly unclear when we are dealing with conventions and when we are dealing with theories about the objective structure of the world. Conventionalism, then, places limits on what can be in the world and on how the world can be structured. So far as I can see, it must be among the most stringent and uncompromising reductive theories in the history of philosophy, therefore." (Nerlich 1976, p. 127)

Conventionalism also has some counter-intuitive implications. As Nerich pointed out, if we are to adopt conventionalism then certain things we generally take to be self evidently objectively true turn out to be mere conventions. For example, I assume that "the Empire State Building is taller than myself" is objectively true regardless of whether I am standing next to it. The point is that the implications of conventionalism contain some hard bullets to bite, and therefore we need some very powerful reasons to subscribe to it.

Nerlich's second objection is that although topological properties can be considered to be the most primitive of all the geometrical properties a figure might possess, it is difficult to see how they may be the only factual ones. The point is that geometrical figures are taken to have metrical properties as well as projective and topological properties, even if we are not considering those properties at the time. In fact, it is difficult, if not impossible, to understand the idea of a figure which has only topological and not projective properties or even metrical ones.

"There is no doubt that we nearly all do think that material objects have definite shapes and lengths, that objects are indeed factually, objectively, equal or unequal in length, whether they are touching or not. If these ideas are 
wrong then it is of the greatest importance to rid ourselves of them since they are widely believed without question.” (Nerlich 1976, p. 128)

"The point being made is that the figure is taken to possess metrical properties both before and after its transformation. But these properties are not included in the projective language since they simply clutter it and distract attention from what is really under the microscope. Of course this doesn't even begin to prove that metrical descriptions are factual or even that they are conceptually basic. But it may make us more curious to discover what argument will have the conclusion that they can't be factual." (Nerlich 1976, p. 131)

The main argument against conventionalism put forward by Nerlich, as I understand it, is essentially as follows: Conventionalism claims that there is no factual difference between the alternative geometric descriptions of space, and that space is determined up to its local topological properties only, and that Euclidean and non-Euclidean geometries differ only by convention. Nerlich claims that this is both counter intuitive and leads us to some undesirable conclusions, such as our inability to make factual comparisons about the relative size of distant objects. The simpler, more intuitive, view is that the alternative description offered to us by geometries are competing theories about the way space actually is, that each geometry describes a different possible world, and it is up to us to determine which one, if any, describes the actual world.

This view seems far more appealing. Even if we admit that Poincare, Hempel and Reichenbach were right, that we can not determine whether space has one geometry, or another geometry with a universal force, surely we are not compelled to conclude that the two theories are factually the same. If two theories having the same observable outcome means that they are factually the same, then we must conclude that a theory that involves angels bending beams of light may be factually the same as relativity theory, which seems absurd. Though we may never know whether it is angels or the force of gravity that bends light rays, I am not comfortable concluding that these two theories differ only by convention. 
Therefore we may conclude that viewing the alternative geometries as different possible worlds leads to a nicer picture of the relationship between mathematical geometry and physical geometry than we get with conventionalism. Once we have concluded that geometries are possible worlds, the question then arises: what are possible worlds? Nerlich asks this very same question. He assumes that they are "not worlds, literally. There is only one world." And he decides that " possible worlds' must be sets of some kind." The reason for this conclusion is that this is the only way he sees for these ideas "to gain intellectual respectability." (Nerlich 1976, p. 138) My claim is that there is another way for these ideas to gain intellectual respectability, and that is to view possible worlds as fictions as described above.

In summary we find that Nerlich's criticisms of Reichenbach's conventionalism has led us back to the conclusion that we drew earlier, that mathematical theories are descriptive of fictional worlds, or rather are descriptive of what an ideal agent may do within fictional worlds. Nerlich has shown that the best theory of geometry is to view alternative geometries as different possible worlds. He does not offer very much in terms of answering how we should view possible worlds except that we should view them in an intellectually respectable way. One very respectable way to view possible worlds is as fictions. Therefore, our view of mathematics fits nicely with Nerlich's solution to the problem of conventionalism. 


\section{$\S 4.5$ Conclusion}

We began this chapter with the aim of developing geometry in a quasi-empirical fashion similar to Kitcher's reformulation of arithmetic. We took as our most primitive operation the operation of constructing straight lines. From here we developed the notion of an incident relation and defined points to be the intersection of two (or more) straight lines. We idealised our ability to perform these operations by considering an ideal agent that is unrestrained by our accidental physical limitations. We then offered P1 $\sim$ P6 as parameters for our ideal agent's ability to perform these line drawing operations. Through investigation we discovered that an ideal agent operating precisely within these parameters can coordinatise the plane and provide a meaningful notion of distance between two points. We thus defined projective geometry to be descriptive of the operations which an ideal agent may perform within the fictional worlds where this agent's fundamental abilities are precisely those outlined above.

In Section 4.3 we considered an analytical model of our synthetic fictional worlds and used it to put further constraints on our ideal agent's ability to perform the line drawing operations involved in transforming one set of lines onto another. We saw that by using matrix algebra to describe geometric transformations, we could easily and precisely state these further constraints. Following the Erlanger programme we showed that by constraining our ideal agent's abilities in various ways we may define collections of possible worlds in which our agent's line drawing operations correspond precisely to Euclidean, hyperbolic or elliptic geometry. Therefore, by considering projective geometry as the super-geometry, we may say that projective geometry is the study of the operations that an ideal agent may perform in any fictional world where these line drawing abilities are in accordance to the axioms we outlined for projective geometry. Furthermore, by considering additional constraints on our agent's ability to use lines to perform transformations, we may conceive of Euclidean, hyperbolic and elliptic geometries as sub-geometries of projective geometry in that they are each the study of the ideal agent's line drawing operations in the set of fictional worlds where the appropriate further constraints have been put on the agent's abilities. 
Finally, when considering the issue of conventionalism as put forward by

Reichenbach, we discovered that the best solution to this problem is to conceive of the different geometries as being descriptive of different possible worlds. This means that the approach we have taken can satisfy the problems raised by Reichenbach in an elegant and decisive way.

We have been able to develop a theory of geometry that contains all the significant features of Kitcher's theory of arithmetic. We have used the operation of drawing lines as our most primitive operation and from this have developed a theory of projective geometry in much the same way that Kitcher developed Mill arithmetic. The advantage of projective geometry is that using only our operation of drawing lines, we may develop a coordinate system for the real projective plane. We may also use the primitive operation of drawing lines to develop the more complicated operation of linear transformations. We may then define certain classes of linear transformations that define the three geometries, Euclidean, hyperbolic and elliptic. Therefore we may develop any geometry from our Kitcherian quasi-empirical foundation.

The significance of the Erlanger programme is that it allows us to apply our quasiempirical foundation to all three geometries. It is also significant in its analytical nature. However we have been able to describe the relationship between the synthetic projective geometry developed in Section 4.2 and the analytical representation of it given by following Klein's approach.

We have also found that if we follow Nerlich's criticisms of Reichenbach's conventionalism, then we will, once again, be led to the conclusion that the most agreeable way to conceive of geometry is as a possible world. Given that we have already concluded that fictionalism is an excellent way to conceive of possible worlds, we see that viewing geometry as a fictional world is a very acceptable theory. 
Chapter 5. Conclusion 


\section{$\S 5.1$ Conclusion}

The aim of this thesis was to find a theory of geometry that will answer the ontological question about the nature of geometrical entities, answer the epistemological question about the nature of geometrical knowledge, fit with the best practices of working mathematicians, and explain the relationship between theoretical geometry and the physical world, explaining the success geometry has in making accurate statements about states of affairs in the physical world. Through the investigation we have discovered that if we view geometry as the idealisation of the activity of drawing lines as carried out by an ideal agent in a fictional world, then we can arrive at a theory of geometry that satisfies all the criteria that we have set down for ourselves. In this Chapter we will briefly review the key points in the investigation, and the key points in our final theory of geometry.

Starting with the epistemological issues we saw that the a priori view held by Hempel is that mathematical statements contain undefined primitive terms and are therefore devoid of factual content and hence incapable of being either true or false. Every mathematical theorem is, according to this view, in fact a conditional statement with conjunction of the axioms as the antecedent, and the theorem as the conclusion. Thus mathematics can only tell us which theorems are implied by which set of axioms, but not which theorems are in fact true.

However, this view presupposes an apsychologistic epistemology. The problem with apriorism is that for a mathematician to know that a theorem is true, all that is required is for the mathematician to believe the theorem to be true, and for the theorem to be logically derivable from the axioms. However, it does not seem reasonable for the mere existence of a proof to be grounds to warrant knowledge of a theorem. In order for a proof to count as justification of the belief in a theorem, the mathematician must be able to go through the psychological process of following the proof. Now, given the long and complicated nature of many mathematical proofs, it seems unreasonable to consider mathematical knowledge to be a priori. Furthermore, what counts as a proof is determined by the academic community, and this changes over time. 
Furthermore, with regard to geometrical knowledge, I see no reason to suppose that we should have a priori knowledge of the geometric nature of physical space. It makes more sense to claim that this knowledge is derived through empirical investigations.

Mill argued for an empirical approach to mathematics claiming that all mathematical statements are based on induction on our experiences dealing with physical objects. Mill`s empiricism, however, lacks the complexity to deal with the more abstract aspects of mathematical knowledge, or the deductive nature of mathematics. Therefore we have taken a Millian approach to the foundations of mathematics and have idealised these foundations to build a quasi-empirical outlook similar to that of Kitcher.

Kitcher's quasi-empirical epistemology is based on two fundamental principles. The first is that mathematical knowledge is passed down from teacher to student in an epistemologically relevant chain from the ancients to today. The second is that the original mathematical knowledge in the ancient world was based on empirical investigations using sense perception, and we may repeat similar experiments in our physical paradigm to help provide a foundation for some of our mathematical knowledge.

This explains the utility of mathematics. Mathematics is useful in providing information and making predictions about states of affairs in the physical world because mathematics is based, ultimately, of empirical investigations. Therefore we may say that mathematical statements are true in virtue of the (structural) nature of the physical world.

On the ontological side we first considered the following line of reasoning as a motivation for Platonism: Given that we have some mathematical knowledge, some mathematical statements must be true, and given that some mathematical statements are true and that our best theory of truth is disquotational, then there must be something in virtue of which these statements are true. Platonism is the theory that mathematical statements are true in virtue of the nature of non-spatio-temporal mind 
independent abstract objects.

Platonism is a widely accepted theory that fits well with the best practices of working mathematicians. However, as Benacerraf pointed out, there must be a causal link between the knower and the objects about which things are known. It is difficult to see how this link may be made if mathematical objects are abstract Platonic entities. The standard response is that this link is attained by something called mathematical intuition. However, this implies that we have a priori knowledge of mathematical entities, something we had already rejected. Moreover, Platonism fails to provide an explanation of why mathematics is useful in making predictions about states of affairs in the real world. A link is needed between ourselves and the abstract objects, ad between the abstract objects and physical reality.

We next considered formalism, the view that mathematical geometry is just the manipulation of meaningless symbols, as an alternative to Platonism. Formalists, such as Hempel, hold the view that the axioms of a mathematical system are devoid of factual content until we assign physical interpretations to the primitive terms. All we can conclude about the truth of mathematical theorems is that any physical interpretation that turns the axioms into true statements will also turn the theorems into true statements. However, with the case of geometry it is not easy to determine if physical interpretations of the axioms are in fact true or not. Also, and more importantly, an explanation of the utility of mathematics is left wanting and so formalism fails to satisfy our desiderata.

Kitcher solved the ontological problem of providing an account of the nature of mathematical entities by moving the focus away from the entities, and onto the operations that mathematicians perform. We consider the fundamental operations that form the foundations of mathematics, and idealise these operations imagining how an ideal agent that is not restrained by our accidental physical limitations may perform these operations. Thus Kitcher views mathematics as being descriptive of the operational activity of an ideal agent.

Kitcher explicitly states that such an ideal agent does not actually exist, however, we can make the notion of the ideal agent, and our ontological commitments, clearer by 
incorporating an element of fictionlism into Kitcher`s quasi-empiricism, thus giving us a more complete theory of geometry. Our ideal agent is considered to be similar in kind to a fictional character, thus, given that we may refer to fictional characters in an unproblematic way, we may also refer to our (non-existent) ideal agent unproblematically. Moreover, fictionalism gives us a way of understanding the plurality of geometries. By considering each geometry to be descriptive of the operational activities of an ideal agent given a certain constraint on their abilities, we may consider each geometry to be descriptive of the fictional worlds in which an ideal agent has those precise abilities. Furthermore, given that we can stipulate the ideal agent's abilities in different ways to generate different geometries, we may consider each geometry to be a particular class of fictional worlds, i.e., all worlds in which an ideal agent has said abilities.

Therefore, at this point we have the outline of a theory of geometry that satisfies all our desiderata. Given that mathematics is useful, the simplest explanation is that mathematics is the idealisation of certain empirical investigations, hence the quasiempirical approach. To avoid the ontological problems of Platonism, we have focused on mathematical operations rather than entities, and idealised these operations. Finally, we have made the nature of these idealisations clear by taking them to describe certain classes of fictional worlds.

Our next step was to actually apply this theoretical framework to geometry in a more precise and rigorous fashion. Therefore in Chapter 4 we began to actually develop geometry in the quasi-empirical fictionalist style that the preceding chapters had led us to. In a break from tradition we began by taking the action of drawing a line with a straight edge and pencil as our underlying physical paradigm. We also allowed our agent to continually produce a line however far, and observed that some lines intersect. We defined a point to be the intersection of two (or more) lines. Using the notion of two intersections (points) being distinct as primitive, we defined the notions of three (or more) lines concurring, and three (or more) points collining.

We then defined projective geometry by imposing four axioms on our ideal agent's ability to perform line drawing operations: 
P1) Given any two lines, we may locate exactly one intersection of these two lines.

P2) Given any two intersections (points), we may construct exactly one line that passes through both of these two points.

P3) Through any intersection of two lines, we may construct a third, distinct, line that also passes through the intersection. (This guarantees that each point is incident with at least three lines).

P4) Given any line, we may construct two more lines, the intersection of which is not on that line.

These axioms make no existential claims about geometrical entities, abstract or otherwise. We have merely stipulated what an ideal agent is able to do.

We also imposed a form of Fano`s axiom, P5, stating that it is not possible for our ideal agent to construct a line joining the diagonal points of a complete quadrangle, and finally we imposed the theorem of Pappus, P6, stating that if the ideal agent draws a hexagon such that the alternate vertices lie on two lines, then a line may be drawn through the intersections of the opposite sides.

We thus define projective geometry to be the study of what an ideal agent may accomplish by carrying out line drawing operations within the parameters stipulated by P1 P6. Thus we are considering projective geometry to be the study of all fictional worlds in which a line drawing agent has abilities that conform to P1 P6. Anything that can be done in all such fictional worlds will be said to be a theorem of projective geometry. The two most interesting results that we discovered is that in a projective world, we may coordinatise the plane using a system of homogeneous coordinates, and we may adopt a notion of measuring distance that is derived from using straight lines to make projections.

Taking the system of homogeneous coordinates that we developed out of an ideal agent's ability to draw lines, we then looked on the analytical side of geometry. We saw that we may correlate the theorems that hold in the purely analytical model of projective geometry with the theorems that hold in our purely synthetic fictional worlds. This frees us to draw on the results of linear algebra and apply then 
meaningfully to geometry.

We then followed the Erlanger programme and considered the properties that are invariant under various classes of transformations. By considering the class of transformations that hold a conic (the absolute) invariant, we may develop either Euclidean, hyperbolic or elliptic geometry depending on the nature of this conic. Thus by following the Erlanger programme we may build the standard geometries out of a foundation of projective geometry. Projective geometry is in turn founded on idealisations of our actual line drawing activities. Therefore we may say that all geometry has a quasi-empirical foundation.

From our fictionalist perspective what the Erlanger programme shows is that we may constrain our ideal agent's line drawing abilities in several ways. By stipulating a certain set of constraints, the fictional worlds in which the ideal agent operates given these constraints may be considered Euclidean worlds. Given another set of constraints, the worlds in which the ideal agent operates may be called hyperbolic worlds. And finally, a further set of constraints gives us elliptic worlds. Therefore each of the main geometries may be viewed as the class of fictional worlds in which an ideal agent may perform operations subject to a certain set of constraints.

Having developed a theory of the nature of geometry we finally looked at the problem of conventionalism to see if our theory can shed some light on this issue. We saw that Nerlich had used the Erlanger programme as a way of describing Reichenbach`s conventionalism. The conventionalist's view is that we can determine the geometric nature of physical space up to its projective properties, or rather its local topological properties, and any further features are only a matter of definitions. Therefore we cannot meaningfully ask whether physical space is Euclidean, hyperbolic or elliptic.

Conventionalism leads to some startling and counterintuitive conclusions. For example, I believe it to be factually true that the Empire State Building is taller than I am, however, according to conventionalism, this is only true as a matter of convention. The more appealing notion, as put forward by Nerlich, is that our various geometric theories offer different competing views of the way the world might be by describing different possible worlds. This conclusion conforms nicely to the nature of 
mathematics as we have described it.

However, the question that drove the conventionalists, "what is the geometric nature of physical space?" remains unanswered. The conventionalists might be right: we may not be able, in principle, to determine the particular geometrical theory to which the physical world conforms. We can, however, state that if our physical limitations were removed, and our ability to draw lines was given in a certain way, then the geometric nature of the physical world would be of a certain kind. 


\section{Bibliography}

Benacerraf, Paul (1965) "What Mathematical Numbers Could Not Be" Philosophical Review 74 (1965): 47-73

Benacerraf, Paul (1998) "What Mathematical Truth Could Not Be" in The Philosophy of Mathematics Today edited by Matthias Schirn, Clarendon Press, Oxford.

Beth, Evert W. (1965) Mathematical Thought: An Introduction to the Philosophy of Mathematics D Reidel Publishing Company, Dordrecht, Holland.

Burgess, John P. and Rosen, Gideon (1997) A Subject with no Object Clarendon Press, Oxford.

Carnap, Rudolf (1964) “The Logicist Foundations of Mathematics" in Philosophy of Mathematics: Selected Readings Edited by Paul Benacerraf and Hilary Putnam, Prentice Hall Inc., New Jersey.

Carnap, Rudolf (1995) An Introduction to the Philosophy of Science Dover, New York

Castaneda, Hector-Neri (1964) "Arithmetic and Reality" in Philosophy of Mathematics: Selected Readings Edited by Paul Benacerraf and Hilary Putnam, Prentice Hall Inc., New Jersey.

Clifford, William Kingdom (1956) "The Postulates of the Science of Space" in -Volume One of The World of Mathematics pp. 552-567, Simon and Schuster, New York.

Chihara, Charles (1998) The Worlds of Possibility Clarendon Press, Oxford.

Coxeter, H. S. M. (1969) Introduction to Geometry Second Edition John Wiley \& Sons, Inc, New York. 
Cremona, Luigi (1913) Elements of Projective Geometry Translated by Charles Leudesdorf, the Carendon Press, Oxford.

Field, Hartry (1989) Realism, Mathematics and Modality Basil Blackwell, Oxford.

Gasking, D. A. T. (1964) "Mathematics and the World" in Philosophy of Mathematics: Selected Readings Edited by Paul Benacerraf and Hilary Putnam, Prentice Hall Inc., New Jersey.

Grabiner, Judith V. (1986) "Is Mathematical Truth Time-Dependent?" in New Directions in the Philosophy of Mathematics: An Anthology edited by Thomas Tymoczko, Birkhauser, Boston.

Hempel, Carl G. (1945) "Geometry and Empirical Science” The American Mathematical Monthly, Vol. 52, No. 1 (Jan., 1945), 7-17 Menasha, Wisc.

Hempel, Carl G. (1964) “On the Nature of Mathematical Truth” in Philosophy of Mathematics: Selected Readings second ed. Pp. 366-381 Edited by Paul Benacerraf and Hilary Putnam, Prentice Hall Inc., New Jersey.

Hersh, Reuben and Davis, Philip J. (1981) The Mathematical Experience Birkhauser, Boston

Hersh, Reuben (1986) "Some proposals for Reviving the Philosophy of Mathematics" in New Directions in the Philosophy of Mathematics: An Anthology edited by Thomas Tymoczko, Birkhauser, Boston.

Heyting, Arend (1964) "The Intuitionist Foundations of Mathematics" in Philosophy of Mathematics: Selected Readings Edited by Paul Benacerraf and Hilary Putnam, Prentice Hall Inc., New Jersey.

Hilbert, D and Cohn-Vossen, S (1952) Geometry and the Imagination Chelsea Publishing Company, New York. 
Hinckfuss, Ian (1975) The Existence of Space and Time Clarendon Press, Oxford.

Jammer, Max (1954) Concepts of Space: The History of Theories of Space in Physics Harvard University Press, Cambridge, Massachusetts.

Kant (1768) "Concerning the Ultimate Foundations of the Differentiation of Regions in Space" in Kant 1968 Selected Pre-Critical Writings and Correspondence with Beck Manchester University Press Barnes \& Noble, New York.

Katz, Victor J. (1998) A History of Mathematics Addison-Wesley, Massachusetts.

Kitcher, Philip (1978) “The Plight of the Platonist” in Nous 12 (1978) pp. 119 - 36 Indiana University.

Kitcher, Philip (1984) The Nature of Mathematical Knowledge Oxford University Press, New York.

Kline, Morris (1953) Mathematics in Western Culture Penguin Books, Middlesex, England.

Kline, Morris (1956) "Projective Geometry" in Volume One of The World of Mathematics With commentaries and notes by James R. Newman, pp. 622-41 Simon and Schuster, New York.

Lakatos, Imre (1986) "A Renaissance of Empiricism in the Recent Philosophy of Mathematics" in New Directions in the Philosophy of Mathematics: An Anthology edited by Thomas Tymoczko, Birkhauser, Boston.

Lehman, Hugh (1979) Introduction to the Philosophy of Mathematics Rowman and Littlefield, Totowa, N.J.

Maddy, Penelope (1990) Realism in Mathematics Clarendon Press, Oxford. 
Maddy, Penelope (1997) Naturalism in Mathematics Clarendon Press, Oxford.

Mill, John Stuart (1950) Philosophy of Scientific Method Hafner Publishing Company, New York.

Nerlich, Graham (1976) The Shape of Space Cambridge University Press, Cambridge.

Poincare, Henri (1964) “On the Nature of Mathematical Reasoning” in Philosophy of Mathematics: Selected Readings second edition, Edited by Paul Benacerraf and Hilary Putnam, Prentice Hall Inc., New Jersey.

Putnam, Hilary (1986) "What is Mathematical Truth?" in New Directions in the Philosophy of Mathematics: An Anthology edited by Thomas Tymoczko, Birkhauser, Boston.

Ray, Christopher (1991) Time, Space and Philosophy Routledge, London.

Redei, L. (1968) Foundation of Euclidean and Non-Euclidean Geometries Pergamon Press, Oxford.

Reichenbach, Hans (1957) The Philosophy of Space and Time Dover Publications Inc., New York.

Robinson, Gilbert (1959) The Foundations of Geometry University of Toronto Press, Toronto.

Rosen, Gideon (1990) "Modal Fictionalism" in Mind, Vol. 99 July 1990. Oxford University Press, Oxford.

Russell, Bertrand (1956) An Essay on the Foundations of Geometry Dover Publications Inc., New York.

Schirn, Matthias (1998) Introduction to The Philosophy of Mathematics Today edited by Matthias Schirn, Clarendon Press, Oxford. 
Seidenberg, A. (1962) Lectures in Projective Geometry D. Van Nostrand Company, Inc. Princeton, New Jersey.

Thom, Rene (1986) "Modern Mathematics An Educational and Philosophical Error?" in New Directions in the Philosophy of Mathematics: An Anthology edited by Thomas Tymoczko, Birkhauser, Boston.

Todd, J. A. (1947) Projective and Analytical Geometry Sir Isaac Pitman \& Sons, Ltd., London.

Torretti, Roberto (1984) Philosophy of Geometry from Riemann to Poincare Reidel Publishing Company, Dordrecht, Holland.

Tuller, Annita (1967) A Modern Introduction to Geometries D. Van Nostrand Company Inc., New Jersey.

Tymoczko, Thomas (1986) "Challenging Foundations" in New Directions in the Philosophy of Mathematics: An Anthology edited by Thomas Tymoczko, Birkhauser, Boston.

Von Neumann, Johann (1964) "The Formalist Foundations of Mathematics" in Philosophy of Mathematics: Selected Readings Edited by Paul Benacerraf and Hilary Putnam, Prentice Hall Inc., New Jersey.

Veblen, Oswald (1910) Projective Geometry Ginn and Company, Boston. 